\title{
Advanced Measurement and Modeling Techniques for Improved SOFC Cathodes
}

\author{
Final Report \\ Reporting Period: 10/01/02-12/31/06 \\ Principle Authors \\ Stuart Adler (PI), L. Dunyushkina, S. Huff, Y. Lu, J. Wilson. \\ Report Issue Date: March 31, 2007 \\ DOE Award Number: DE-FC26-02NT41566 \\ Reporting Organization: \\ University of Washington \\ Box 351750 \\ Seattle, WA 98195-1750
}

DOE Project Manager: Lane Wilson

tel 304-285-1336, email lane.wilson@netl.doe.gov 


\section{Disclaimer}

This report was prepared as an account of work sponsored by an agency of the United States Government. Neither the United States Government not any agency thereof, nor any of their employees, makes any warranty, express or implied, or assumes any legal liability or responsibility for the accuracy, completeness, or usefulness of any information, apparatus, product, or process disclosed, or represents that its use would not infringe privately owned rights. Reference herein to any specific commercial product, process, or service by trade name, trademark, manufacturer, or otherwise does not necessarily constitute or imply its endorsement, recommendation, or favoring by the United States Government or any agency thereof. The views and opinions of authors expressed herein do not necessarily state or reflect those of the United States Government or any agency thereof. 


\begin{abstract}
The goal of this project was to develop an improved understanding of factors governing performance and degradation of mixed-conducting SOFC cathodes. Two new diagnostic tools were developed to help achieve this goal: 1) microelectrode half-cells for improved isolation of cathode impedance on thin electrolytes, and 2) nonlinear electrochemical impedance spectroscopy (NLEIS), a variant of traditional impedance that allows workers to probe nonlinear rates as a function of frequency. After reporting on the development and efficacy of these tools, this document reports on the use of these and other tools to better understand performance and degradation of cathodes based on the mixed conductor $\mathrm{La}_{1-\mathrm{x}} \mathrm{Sr}_{\mathrm{x}} \mathrm{CoO}_{3-\delta}$ (LSC) on gadolinia or samaria-doped ceria (GDC or SDC). We describe the use of NLEIS to measure $\mathrm{O}_{2}$ exchange on thin-film LSC electrodes, and show that $\mathrm{O}_{2}$ exchange is most likely governed by dissociative adsorption. We also describe parametric studies of porous LSC electrodes using impedance and NLEIS. Our results suggest that $\mathrm{O}_{2}$ exchange and ion transport co-limit performance under most relevant conditions, but it is $\mathrm{O}_{2}$ exchange that is most sensitive to processing, and subject to the greatest degradation and sample-to-sample variation. We recommend further work that focuses on electrodes of well-defined or characterized geometry, and probes the details of surface structure, composition, and impurities. Parallel work on primarily electronic conductors (LSM) would also be of benefit to developers, and to improved understanding of surface vs. bulk diffusion.
\end{abstract}




\section{Table of Contents}

Executive Summary $\quad 5$

Motivation and Scope $\quad 6$

Part I: Advanced Measurement and Modeling Tools $\quad 8$

Section A: Microelectrode Half-Cells $\quad 8$

A-1. Introduction $\quad 8$

A-2. Microelectrode Cell Design $\quad 10$

A-3. Development of Insulating Mask 11

A-4 Validation Studies Using Pt and LSC Electrodes 13

A-5 Results and Discussion 15

A-6 Summary 19

Section B: Nonlinear Impedance Spectroscopy (NLEIS) 20

$B-1$. Introduction $\quad 20$

B-2. NLEIS Apparatus and Procedures 20

B-3. Signal Acquisition and Analysis 21

B-4. Test-Bed Application \#1: Porous LSC on SDC 26

B-5 Test-Bed Application \#2: Mass Transfer Effects in SOFC Tests 28

B-6 Test-Bed Application \#3: Grain Boundary Effects in Oxide Electrolytes 33

B-7 Summary 36

Part II: Studies of Reaction and Degradation Mechanisms Governing 37

$\mathrm{La}_{1-\mathrm{x}} \mathrm{Sr}_{\mathrm{x}} \mathrm{CoO}_{3-\mathrm{d}}$ (LSC) on Gd- and Sm- doped Ceria.

Section C: Studies of Thin Film LSC Electrodes 37

C-1. Introduction $\quad 37$

C-2. Experimental 38

C-3. Film Characterization 38

C-4. Linear Response 39

C-5. Nonlinear Response $\quad 40$

C-6. Modeling $\quad 42$

C-7. Discussion $\quad 45$

C-8. Summary/Conclusions 46

Section D: Parametric Studies of Porous LSC electrodes 47

D-1. Introduction $\quad 47$

D-2. Fabrication of Porous LSC electrodes on SDC. 48

D-3. Microstructural Characterization. $\quad 50$

D-4. Elemental Analysis $\quad 56$

D-5. Electrochemical Testing 56

D-6. Post-Experimental Data Analysis $\quad 59$

D-7. Evidence for Co-limitation and Dissociative Adsorption 60

D-8. Factors Influencing Interfacial Resistance 65

D-9. Summary $\quad 69$

$\begin{array}{ll}\text { References } & 70\end{array}$ 


\section{Executive Summary}

SOFC cathodes based on perovskite mixed conductors offer one possible route to improved performance at reduced temperature. While these materials are promising, they have not been widely adopted by SOFC developers because they react unfavorably with YSZ-based electrolytes, often exhibit unpredictable performance, and suffer from long-term degradation. The goal of this FE-sponsored project has been to develop an improved understanding of the factors governing performance and degradation of mixed-conducting SOFC cathodes, and in so doing, provide new ideas and diagnostic tools that may aid developers in bringing these (or other) electrodes to fruition.

This report is divided into Part I \& Part II. Part I (Sections A \& B) describes two new diagnostic tools developed under DOE sponsorship for probing SOFC cathode performance. The first tool (described in Section A) is the use of microelectrode half-cells for improved isolation of cathode impedance on thin electrolytes. We show that this method permits accurate isolation of overpotential and impedance under polarized conditions, and enhances throughput of electrochemical measurements. A second tool developed under this project (described in Section B) is nonlinear electrochemical impedance spectroscopy (NLEIS), a variant of traditional impedance that allows workers to probe rate nonlinearity as a function of frequency. We demonstrate the ability of NLEIS to isolate nonlinearities in several test-bed applications, including kinetics (porous SOFC cathodes), ion conduction (grain-boundary conductivity of ceria-gadolinia), and gas-phase mass-transfer (discovered during measurements of SOFC buttoncell performance at Pacific Northwestern National Laboratory (PNNL)).

Part II (sections $\mathrm{C} \& \mathrm{D}$ ) describes more specifically on our progress in understanding performance and degradation of cathodes based on the mixed conductor $\mathrm{La}_{1-\mathrm{x}} \mathrm{Sr}_{\mathrm{x}} \mathrm{CoO}_{3-\delta}$ (LSC) on gadolinia and samaria-doped ceria (GDC and SDC). In Section C we describe the use of NLEIS to measure $\mathrm{O}_{2}$ exchange on thin-film LSC electrodes. We show that by combining a welldefined electrode geometry with nonlinear rate analysis (NLEIS), the full nonlinear rate law for $\mathrm{O}_{2}$ oxidation/reduction could be isolated from other factors such as transport or electrode morphology. By comparing the measured kinetics to thermodynamically self-consistent kinetic models, we show that the $\mathrm{O}_{2}$ reduction reaction is likely to be limited by dissociative adsorption on limited unsaturated surface vacancy sites. Implications of this rate law (and it's interpretation) for materials selection/development are discussed, and then applied to porous electrodes (Section D). In Section D we describe a series of parametric studies of impedance (and some NLEIS) measurements of porous LSC electrodes over a wide range of processing conditions (electrode firing temperature, electrolyte substrate preparation, Sr-doping) and operating conditions ( $T, P_{O 2}$, polarization, and time). These studies suggest the following:

- Unlike LSC on YSZ, at sufficiently high firing temperatures $\left(\geq 1000^{\circ} \mathrm{C}\right)$, LSC electrodes on GDC/SDC exhibit little or no interfacial resistance - i.e. charge-transfer is reversible between electrode and electrolyte materials. This appears to be a unique feature of these electrodes, as distinct from more traditional materials where charge-transfer at the TPB is dominant (LSM/YSZ). Pre-polishing of the electrolyte prior to electrode firing increases interfacial resistance, suggesting that impurities or grain-boundary phases present on the electrolyte may be important in establishing a reversible bond during electrode processing. 
- Given a reversible LSC/ceria interface, performance of LSC electrodes appears to be colimited by $\mathrm{O}_{2}$ reduction on LSC and ionic transport on/within LSC to the electrolyte. This conclusion is supported by a strong $2^{\text {nd }}$-order correlation between electrode frequency and electrode resistance that spans a wide range of conditions $\left(P_{O 2}, T\right.$, Sr-doping, time, polarization). This correlation breaks down at higher temperatures $\left(\mathrm{T} \geq 725^{\circ} \mathrm{C}\right)$, which may reflect a shift toward a more purely bulk-diffusion-limited reaction. This view is further supported by NLEIS results, which show much weaker $2^{\text {nd }}$ and $3^{\text {rd }}$ harmonics than predicted based on a co-limited model under these conditions.

- Above a firing temperature of $\sim 1000^{\circ} \mathrm{C}$, electrode performance degrades significantly with increased firing temperature. BET measurements, as well as recent analysis of the electrode morphology using FIB-SEM show only minor changes in morphology over the same range of processing conditions. Quantitative analysis of electrode frequency and resistance as a function of firing conditions shows that these changes are most likely related to the changes in kinetics at the surface. Some reasons why the surface chemistry might be negatively influenced by increased firing temperature are discussed.

- Examination of electrode frequency and resistance as a function of time suggests that the primary contributor to electrode degradation are changes to the $\mathrm{O}_{2}$ exchange rate at the surface. Likewise, significant sample-to-sample variation in performance also appears to be tied most closely to differences in $\mathrm{O}_{2}$ exchange rate. Thus (as seen with firing conditions), it appears to be the surface chemistry that is the most sensitive factor in governing performance. It remains unclear what changes are occurring to the surface on an atomic level, or why these changes are so sensitive to the details of processing and operation. We recommend further work that focuses on electrodes of well-defined or characterized geometry, and probes the details of surface structure, composition, and impurities.

Based on these results, we recommend further work that focuses on electrodes of welldefined or characterized geometry, and probes the details of surface structure, composition, and impurities. Parallel work on primarily electronic conductors (LSM) would also be of benefit to developers, and to improved understanding of surface vs. bulk diffusion. 


\section{Motivation and Scope}

The high operating temperature of current state of the art SOFC cathode materials $\left(>800^{\circ} \mathrm{C}\right)$ limits performance and cost reduction efforts. Many recent alternatives to LSM/YSZ incorporate mixed conducting ceramics (materials which carry both oxygen ions and electrons) in order to substantially improve the activity and selectivity of the electrode reaction at reduced temperature. While these materials are promising, they have not been widely adopted by SOFC developers because they react unfavorably with YSZ-based electrolytes, often exhibit unpredictable performance, and suffer from long-term degradation problems. The overall goal of this DOE/FE-sponsored project has been to develop an improved understanding of the factors governing performance and degradation of mixed-conducting SOFC cathodes, and in so doing, provide new ideas and diagnostic tools that may aid developers in bringing these (or other) electrodes to fruition.

Under sponsorship of this program, two new diagnostic tools have been developed for probing SOFC cathode performance. The first of these is the use of microelectrode half-cells for improved isolation of cathode impedance on thin electrolytes. We show that this method permits accurate isolation of overpotential and impedance under polarized conditions, and enhances throughput of electrochemical measurements. A second tool developed under this program is nonlinear electrochemical impedance spectroscopy (NLEIS), a variant of traditional impedance that allows workers to resolve nonlinear rates as a function of frequency. We demonstrate the ability of NLEIS to isolate nonlinearities associated with various sub-steps in the overall $\mathrm{O}_{2}$ reduction reaction, and thus provide insight regarding the electrode mechanism.

The techniques described above were applied to better understand the performance and degradation of cathodes based on the mixed conductor $\mathrm{La}_{1-\mathrm{x}} \mathrm{Sr}_{\mathrm{x}} \mathrm{CoO}_{3-\delta}$ (LSC) on gadolinia and samaria-doped ceria (GDC and SDC). In conjunction with a NSF-sponsored project, we examined $\mathrm{O}_{2}$ exchange on thin-film LSC electrodes, allowing us to isolate the $\mathrm{O}_{2}$ exchange reaction on LSC. By combining a well-defined electrode geometry with NLEIS, we were able to map the nonlinear rate law for $\mathrm{O}_{2}$ exchange and thereby determine the most likely atomic-level mechanisms governing $\mathrm{O}_{2}$ dissociation. We also applied microelectrodes, EIS, and NLEIS to the study of porous LSC on SDC over a wide range of processing conditions (electrode firing temperature, electrolyte substrate preparation, Sr-doping) and operating conditions $\left(T, P_{O 2}\right.$, polarization, and time). These studies have allowed us to identify some of the most general and important factors in performance, and narrow the list of possible factors responsible for degradation and sample-to-sample variation.

The remainder of this report is divided into two Parts: I \& II. Part I details the two techniques developed under this program: microelectrodes and NLEIS. Part II details our findings with respect to LSC electrodes on GDC and SDC. 


\section{Part I. Advanced Measurement and Modeling Tools}

\section{A. Microelectrode Half-Cells}

\section{A-1. Introduction}

As shown in Fig. A-1(a), some of the most commonly used 3-electrode cell configurations used for studies of electrode behavior on planar cells involves placement of a reference electrode (RE) coplanar with the working electrode (WE). In principle, this reference electrode is supposed to measure the electrolyte potential along an equipotential surface lying somewhere between the WE and counterelectrode (CE), thus isolating the working-electrode overpotential to the working half-cell, $V_{\mathrm{WE}}-V_{\mathrm{RE}}$.

(a)

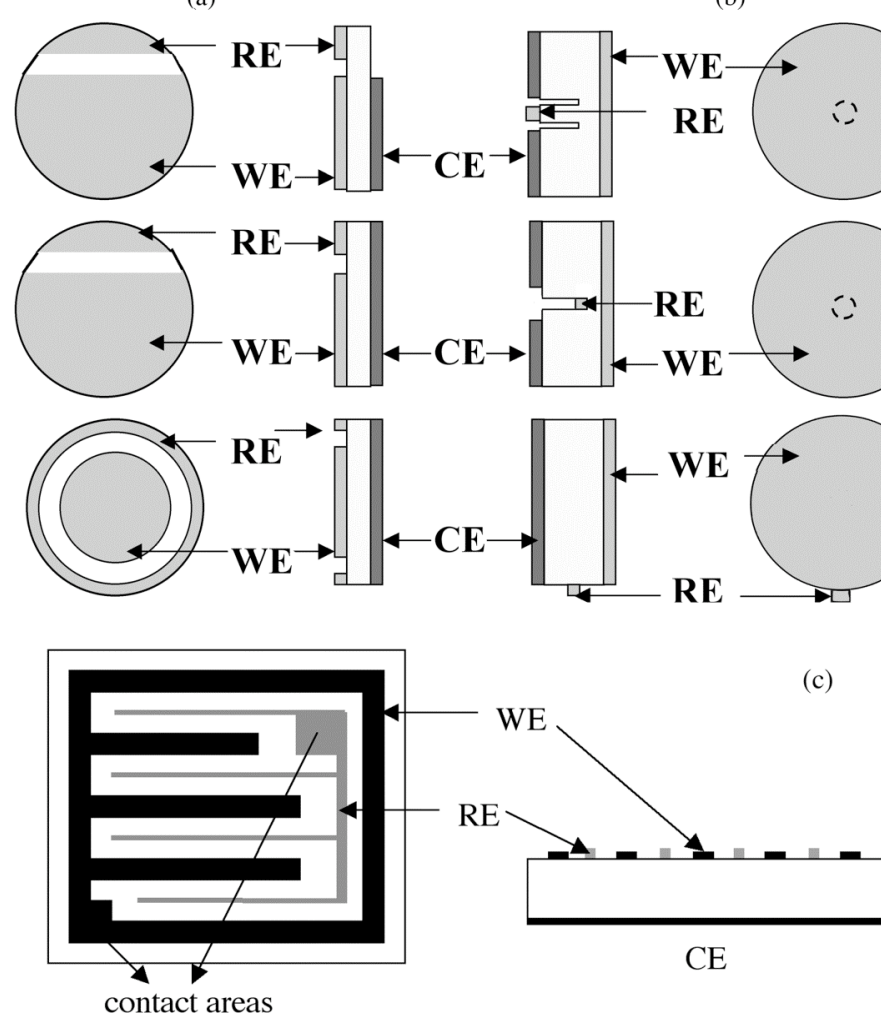

Fig. A-1. Common 3-elecrode configurations for SOFCs.
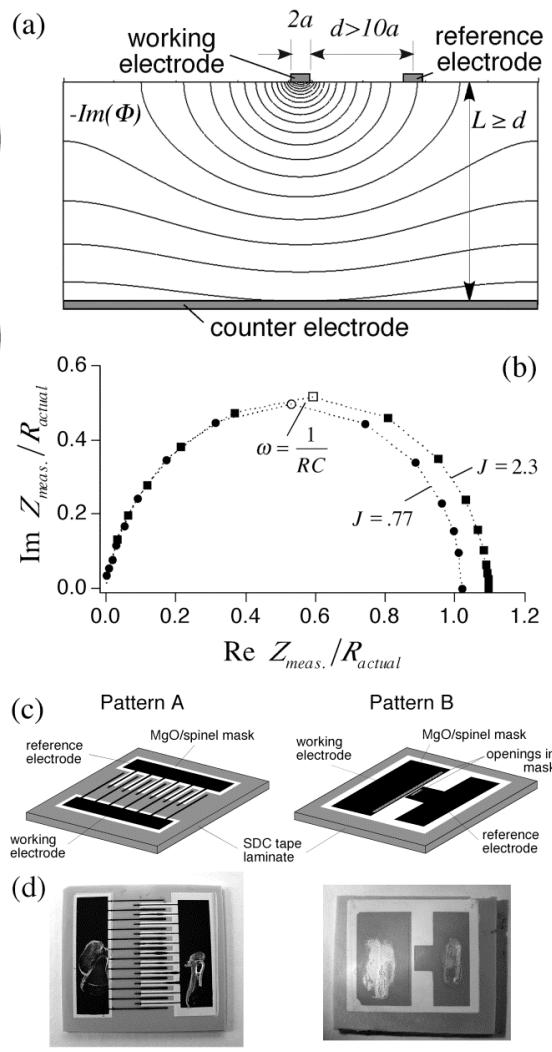

Fig. A-2. Microelectrode design developed under this program.

However, a number of studies ${ }^{[1-5]}$ have shown that the accuracy of 3-probe d.c. polarization and a.c. impedance measurements can be extremely sensitive to the exact details of the cell geometry and position of the RE. Nagata et al. ${ }^{[1]}$ has shown experimentally that the accuracy of d.c. electrode overvoltage measurements depends strongly on symmetrical positioning of the active electrodes on opposite sides of the planar cell, as well as placement of RE far from the edge of the working electrode. The positioning of the electrodes becomes even more critical in 
planar cells with thin $(50-500 \mu \mathrm{m})$ electrolytes, which normally form the basis of planar SOFC technology (owing to their reduced ohmic resistance). Winkler et al. ${ }^{[3]}$ have shown using finite element analysis (FEA) that that the error in determining the electrode polarization resistance in a planar cell rises with an increase in the ratio of the active electrode displacement to the electrolyte thickness.

Adler ${ }^{[4]}$ has further studied the impact of cell geometry and RE positioning on impedance measurements. This study employed FEA to calculate both the real and imaginary parts of potential distribution in a thin planar cell with misaligned active electrodes, thereby simulating half-cell impedance as a function of frequency. According to these calculations, even slight displacement of WE with respect to $\mathrm{CE}(\sim 10 \%$ of the electrolyte thickness) results in significant distortions in the measured electrode impedance, including cross-contamination of the working electrode impedance with frequency characteristics of the counterelectrode.

Given the difficulties of making accurate measurements on planar cells, a number of authors have investigated cell geometries based on thick pellets (Fig. A-1(b)). Hsieh et. al. ${ }^{[2]}$ have shown both experimentally and using numerical models that a preferred solution for accurate studies of electrode performance is an internal reference electrode, which (by symmetry) avoids disturbances to the potential distribution near the WE. FEA calculations by Winkler et. al. ${ }^{[3]}$ and Adler ${ }^{[4]}$ have confirmed these findings. However, in addition to the complexity of fabrication, a significant disadvantage of using a thick pellet is difficultly achieving high current densities, and/or inability to avoid Joule-heating of the sample. Another potential disadvantage of this geometry is that it may require materials and processing conditions that deviate significantly from those used in commercial fabrication of thinner cells.

As an alternative, Hashibon and coworkers ${ }^{[5]}$ have discussed the possibility of using a microelectrode configuration on planar cells. They have suggested placing strips of REs in small gaps between WEs, as shown in Fig. A-1(c). The width of RE as well as the distance between RE and WE should be much smaller than the electrolyte thickness. The contact areas of RE and WE where the leads should be applied are placed on the periphery of the electrodes. It was demonstrated (theoretically) that the suggested geometry ensures insensitivity of RE potential to the exact position of RE relative to WE.

Similarly, Adler ${ }^{[4]}$ has considered a planar cell with the microelectrode configuration shown in Fig. A-2(a). FEA simulations of real and imaginary potential show that this geometry results in a nearly cylindrical potential distribution around the WE, provided the center-to-center distance between WE and RE $(r=d+a)$, obeys $10 a \tilde{<} r \tilde{<} L$, where $2 a$ is a strip width, and $L$ is electrolyte substrate thickness. Under these conditions, this geometry was shown theoretically to provide excellent frequency isolation of the WE response, with an electrode overvoltage containing a relatively undistorted WE overpotential and impedance (Fig. A-2(b)).

As with circular microelectrodes ${ }^{[6]}$, a key feature of the geometry shown in Fig. A-2(a) is that the ohmic resistance of the half-cell is determined by the width of the electrode rather than the thickness of the electrolyte substrate. For the geometry shown in Fig A-2(a), the electrolyte potential drop, $U_{E}$, between the working electrode and a RE lying on the surface a distance $r$ from the center of symmetry of the working electrode can be calculated based on potential theory as ${ }^{[4]}$ : 


$$
\frac{U_{E}}{i}=R_{E}=\frac{2 a}{\pi \sigma} \cosh ^{-1}\left(\frac{r}{a}\right),
$$

where $i$ is the average current density at the WE, and $\sigma$ is electrolyte conductivity. By subtracting $U_{\mathrm{E}}$ from the measured voltage, one can obtain the average WE overpotential. In addition to a small ohmic resistance, other possible benefits of this geometry (relative to a pellet) include ease of fabrication on a planar substrate, small total current (allowing higher current density and less induction effects), and small size (potentially allowing multiple cell tests on one substrate, or multiple cells in a single furnace).

The objectives of this study were to fabricate half-cells with the microelectrode pattern considered in Fig. A-2(a) using standard SOFC materials and techniques, and to determine experimentally how well this cell geometry isolates and quantifies the WE overpotential and impedance. In contrast to Hashibon's geometry (Fig. A-1(c)), where the wire contact area may disturb the potential distribution in the cell, the implementations discussed herein (Figs. A$2(\mathrm{c}, \mathrm{d}))$ involve the use of an insulating mask to isolate the microelectrode contact area from the electrolyte. After development of an appropriate mask material for use on Sm-doped ceria, microelectrode half-cells of $\mathrm{Pt}$ and $\mathrm{La}_{1-\mathrm{x}} \mathrm{Sr}_{\mathrm{x}} \mathrm{CoO}_{3-\delta}$ were fabricated and tested using d.c polarization measurements and a.c. impedance. Results-to-date suggest this technique is promising for isolation and quantification of SOFC half-cell response.

\section{A-2. Microelectrode Cell Design}

Fig. A-2(c) presents two possible implementations of the electrode geometry shown in Fig. A-2(a). In pattern A, the WE consists of a "comb" of long, narrow WE strips, between which short RE strips are placed. An insulating mask (described below) is used to prevent contact of the WE except along the length of these strips. A similar mask is also used to pinpoint the location of the RE contacts in the middle of the WE strips. As long as the current density along the length of each WE strip is uniform, the electrolyte potential distribution (in a cross-section of the cell near the RE contacts) is similar to that shown in Fig. A-2(a). A photo of a pattern A cell is shown in Fig. A-2(d) (left).

One potential drawback of pattern $\mathrm{A}$ is that in the absence of a current collector, we expect the electrical potential to vary along the length of the WE strip unless the electrode has an unusually high electrical conductivity (or is very thick) ${ }^{[7]}$. Applying an effective current collector to a narrow WE strip presents significant fabrication and measurement challenges. As an alternative, we also investigated a second electrode pattern shown in Fig. A-2(c) (pattern B). Rather than patterning the microelectrode, pattern B involves first patterning an insulating mask having openings of well-defined geometry where the WE will make contact to the electrolyte. A macroscopic electrode pattern is then printed over the entire mask, as shown. This pattern avoids polarization losses along the WE strip by providing current radially (from the side, rather than end, of the strip). A photo of a pattern B cell is shown in Fig. A-2(d) (right).

As discussed below, one challenge of pattern $\mathrm{B}$ is that it involves more stringent requirements than pattern $\mathrm{A}$ on the continuity and insulation properties of the mask. The exact area of the WE can also be more difficult to control with pattern B than in the case of pattern A. In the ideal case, the electrode material fills the entire opening in the mask. In reality, the degree of contact depends on the mask thickness and profile of the opening edges. If screen printing is 
used for the electrode deposition, the electrode ink must be viscous enough to avoid leaking and closing of the WE and RE through the narrow gap between them, while not being so viscous as to not fill the gaps completely. Post-mortem SEM analysis of the fractured cell can help to determine the WE area, as discussed in the following section.

\section{A-3. Development of Insulating Mask}

In addition to having very low electrical and ionic conductivity, the mask material must match the electrolyte closely in thermal expansion to avoid delamination. In this study, we used samarium-doped ceria (SDC) of composition $\mathrm{Ce}_{0.8} \mathrm{Sm}_{0.2} \mathrm{O}_{2-\delta}$ as the electrolyte, which has a coefficient of thermal expansion (CTE) of about $12.5 \mathrm{ppm} /{ }^{\circ} \mathrm{C}$. Highly resistive oxides, $\mathrm{MgO}$ and $\mathrm{MgAl}_{2} \mathrm{O}_{4}$ (Alfa-Aesar) were used for preparation of mask. It was calculated (based on a volumetric average) that a physical mixture of $62 \mathrm{wt} \% \mathrm{MgO}$ and $38 \mathrm{wt} \% \mathrm{MgAl}_{2} \mathrm{O}_{4}$ should have a thermal expansion coefficient (CTE) closely matching the CTE of the electrolyte. The CTE data for $\mathrm{MgO}$ and $\mathrm{MgAl}_{2} \mathrm{O}_{4}$ used for this calculation were taken from ${ }^{[8]}$.

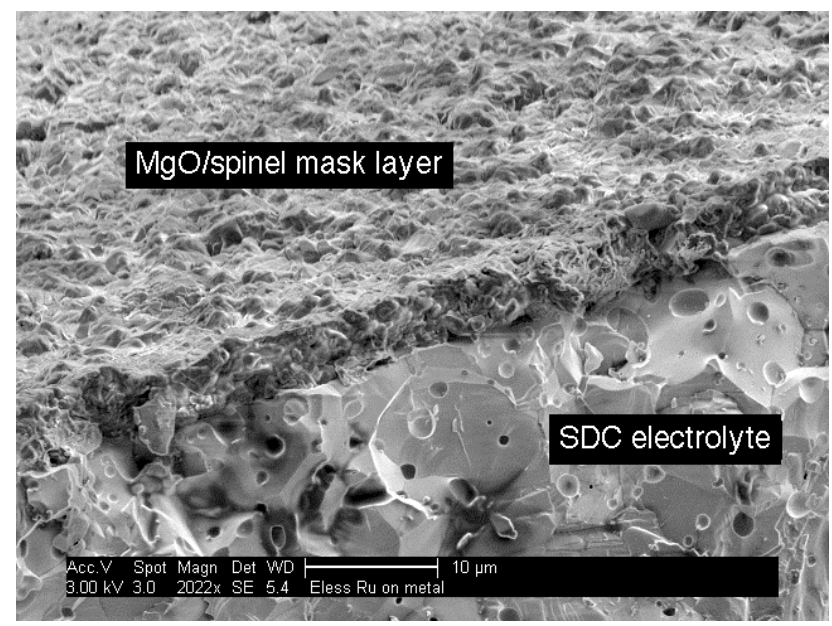

Fig. A-3. SEM micrograph of $\mathrm{MgO} /$ Spinel insulating mask after firing.

The oxide powders were blended into ink containing alpha-terpineol, ethyl cellulose, and oleic acid using a 3-roll mill. This ink was then patterned onto the SDC substrate by screenprinting using 325 or 400 mesh-count stainless steel screens. The printed mask was sintered at $1450^{\circ} \mathrm{C}$ for 4 hours. Depending on the screen quality and snap-off, the screenprinting deposition produces a 5-10 $\mu \mathrm{m}$ thick film mask on the surface of the SDC substrate. The morphology and thickness of the insulating mask was characterized using SEM (FEI Sirion). As an example, Fig. A-3 shows a fracture cross-section of a 5- $\mu \mathrm{m}$ thick mask on the surface of SDC. The match in the CTE between this film and the SDC was sufficiently good that the film remained welladhered to the electrolyte over the $25-1450^{\circ} \mathrm{C}$ range.

The electrical properties of the mask were studied by two-probe a.c. impedance, using a Solartron 1287 potentiostat and 1252A frequency response analyzer. One or multiple layers of the mask ink were printed and sintered onto a dense SDC substrate. Cathode materials of interest 
were then printed on top of the mask layer, as well as on the opposite side of the cell (directly on the surface of SDC opposite the masked electrode). We tested two different electrodes materials: Pt and LSC-82, made with the same electrode inks and firing conditions as for working halfcells. Silver wires were attached to the electrodes using silver paste and, in the case of LSC-82, a porous silver current collector. The measurements were conducted at $700-750^{\circ} \mathrm{C}$ in air.

The impedance of the mask layers generally exhibited a purely semicircular response, with a resistance in the range of 5000-150000 $\Omega / \mathrm{cm}^{2}$, depending on thickness and firing conditions. The estimated capacitance of the mask layer was between $2 \times 10^{-10}$ and $5 \times 10^{-10} \mathrm{~F}$, which is consistent with the mask thickness (5-25micons), porosity (20-30\%) and dielectric constant of $\mathrm{MgO}$ and $\mathrm{MgAl}_{2} \mathrm{O}_{4}(\varepsilon \approx 9.5)$. One special case observed with LSF-82 electrodes was a gradual decrease of the mask resistance from $250 \mathrm{kOhm}-\mathrm{cm}^{2}$ to $25 \mathrm{kOhm}-\mathrm{cm}^{2}$ during the first 100 hours at $750^{\circ} \mathrm{C}$. We currently speculate this was related to penetration of $\mathrm{Ag}$ from the current collector into the pores of the mask layer.

In order to estimate the effect of finite mask resistance on the a.c. impedance response of pattern-B half-cells, we constructed a 3-D finite-element model of the complex potential distribution in the electrolyte of a pattern B half-cell (solved using FEMLAB 2.2 and MATLAB 6.1 software). The method applied followed precisely that of reference ${ }^{[4]}$, where the cell geometry was taken to be that shown in Fig. A-2(c), pattern B, and the mask and working electrode were assumed to have linear RC characteristics. These calculations showed that a mask resistance of $>25 \mathrm{kOhm}-\mathrm{cm}^{2}$ results in negligible error or distortion of the measured microelectrode impedance ${ }^{[7]}$.

One challenge we encountered during processing of the mask was establishing uniform thickness and porosity sufficient to achieve the required resistances calculated above. A common defect observed in single mask layers was an isolated region where the mask becomes too thin and porous, such that the electrode material is able to penetrate the pores of the mask, and make contact to the electrolyte ${ }^{[7]}$. In practice, we found that two mask layers (printed and fired separately), were required to achieve reproducible mask resistances resistance $\geq 25 \mathrm{k} \Omega-\mathrm{cm}^{2}$ (while a single layer often had much lower resistance, $\sim 5 \mathrm{k} \Omega-\mathrm{cm}^{2}$, with more manufacturing variability). One possible explanation is that the location of isolated defects are statistically random, and thus the second layer tends to cover defects of the first layer while introducing new defects in places that are already covered.

The requirement for two or more printed mask layers has unfortunate consequences for the remaining processing of the cell. One consequence is a thicker mask layer ( $>10$ microns), which can interfere with the screenprinting of the electrode ink. Near the edge of the mask, the electrode printing can be wider than the screen pattern and thickened because the mask prevents the electrode screen from sitting flush with the electrolyte surface ${ }^{[7]}$. In addition, the requirement for two mask layers also requires that the registration (lateral position) of the second layer relative to the first be very accurate (microns), otherwise the second layer will occlude the WE and RE openings in the first layer. In practice, we found registration with our hand-operated screen printer to be very difficult. Together, these difficulties generally caused mask openings to be much narrower than the designed screen pattern $(150 \mu \mathrm{m})$. Typical values were 35 100 microns. As described below, this variability made assessment of the electrode area (and ohmic resistance) difficult - a problem that needs to be tacked before the technique can be fully quantitative. 
As an alternative to printing two mask layers, we also tried to fabricate thinner, more dense masks by using a higher firing temperature. Firing at $1500^{\circ} \mathrm{C} \sim 1550^{\circ} \mathrm{C}$ resulted in a visually denser film (based on SEM). However the insulating properties of single-layer masks processed at higher temperature is generally unsatisfactory. This is likely related to the presence of small defects in the film, which exist even if the film is denser overall. We also observed a reaction between the mask and ceria, which becomes more serious at higher temperatures ${ }^{[7]}$.

\section{A-4 Validation Studies Using Pt and LSC Electrodes}

Microelectrode half-cells were fabricated using laminated SDC substrates and various cathode materials including $\mathrm{Pt}, \mathrm{La}_{0.8} \mathrm{Sr}_{0.2} \mathrm{CoO}_{3-\delta}$ (LSC-82) and $\mathrm{La}_{0.3} \mathrm{Sr}_{0.7} \mathrm{CoO}_{3-\delta}$ (LSC-37). As explained elsewhere ${ }^{[7]}$, pattern $\mathrm{B}$ was chosen for the perovskite oxide electrodes. The design width of the WE for both pattern A and pattern B were the same: $2 a=150 \mu \mathrm{m}$ (although the actual width varied considerably from the design width for reasons discussed below). The length of the WE strips in pattern A was $1.0 \mathrm{~cm}$, while somewhat longer $(1.25 \mathrm{~cm})$ for pattern $\mathrm{B}$. These dimensions resulted in a design area of $0.15 \mathrm{~cm}^{2}$ and $0.19 \mathrm{~cm}^{2}$ for patterns A and B, respectively. Finally, the distance $d$ between the WE edge and the center of the RE was $0.6 \mathrm{~mm}$ in pattern A and $1.0 \mathrm{~mm}$ for pattern $\mathrm{B}$.

Table A-1. Nomenclature and configuration of cells

\begin{tabular}{|l|l|}
\hline \multicolumn{1}{|c|}{ Name } & \multicolumn{1}{c|}{ Cell configuration } \\
\hline Pt(sym) & Geometrically symmetric cell with identical Pt electrodes \\
\hline Pt(A) & Microelectrode half-cell with WE, RE and CE made of Pt; pattern A \\
\hline LSC-82(sym) & Geometrically symmetric cell with identical LSC-82 electrodes \\
\hline LSC-82(B) & Microelectrode half-cell with WE, RE and CE made of LSC-82; pattern B \\
\hline 37/SDC/82 & Geometrically symmetric cell with opposing LSC-37 and LSC-82 electrodes \\
\hline LSC-37(B) & Half-cell with WE and RE made of LSC37; CE made of LSC82; pattern B \\
\hline
\end{tabular}

As summarized in Table A-1, three configurations of microelectrode half-cells were prepared: 1) pattern-A cells with $\mathrm{WE}, \mathrm{RE}$ and $\mathrm{CE}$ made of $\mathrm{Pt}$; 2) pattern-B cells with WE, RE and CE made from LSC-82; and 3) pattern-B half-cells with LSC-37 as WE and RE, and LSC-82 as the counter electrode. Geometrically symmetric cells (with two full-sized $\sim 1 \mathrm{~cm}^{2}$ active electrodes) were also fabricated, using the same materials and techniques as for the half-cells. 
These cells included symmetric cells with identical electrodes, Pt/SDC/Pt and LSC82/SDC/LSC-82, and cells of symmetric geometry but with the electrodes of different composition, LSC-37/SDC/LSC-82. For convenience, each of these configurations have abbreviated names, which are also summarized in Table A-1.

The SDC electrolyte $\left(\mathrm{Ce}_{0.8} \mathrm{Sm}_{0.2} \mathrm{O}_{2-\delta}\right.$ plus $1 \% \mathrm{TiO}_{2}$ as a sintering aid) was processed and characterized as follows. Green tape of thickness 300 600 $\mu \mathrm{m}$ (Ceramatec, Salt Lake City) was cut into rectangular plates of approximately $2.5 \mathrm{~cm} \times 2.5 \mathrm{~cm}$, which were then stacked and heatlaminated to obtain a desired fired thickness. Lamination was accomplished by hot-pressing the green tapes together after wetting with toluene. The laminated plates were fired at $1650^{\circ} \mathrm{C}$ in a dedicated Deltech furnace. For microelectrode half-cells, final thickness was $\sim 1000$ microns; for symmetric cells, 250-500 microns. SEM (FEI Sirion) of the electrolyte cross section showed that the plates were dense and uniform across the thickness. XRD characterization (Philips 1820 $\mathrm{XRD}$ ) of the electrolyte showed presence of a single $\mathrm{CeO}_{2}$-based phase. Conductivity of laminated and non-laminated SDC samples was measured via the d.c. four-probe technique, using Pt wire leads attached to the sample with Pt paste.

The mask layers (for pattern A and B) were processed as described previously. RE and WE patterned electrodes were applied to the masked SDC substrate using screenprinting, and then fired in a tube furnace (Lindberg/Blue M). For Pt electrodes we used commercial Pt ink (A3788A, Engelhard). For LSC-82 and LSC-37, we made our own screenprinting inks from nominally phase-pure LSC powder (Superconductive Components, Inc., Columbus), made as described below for $\mathrm{MgO} /$ spinel. The $\mathrm{CE}$ was subsequently screen-printed and fired onto the opposite surface of the electrolyte. Electrode firing temperatures were 900,1080 and $1250^{\circ} \mathrm{C}$ for Pt, LSC-82 and LSC-37, respectively.

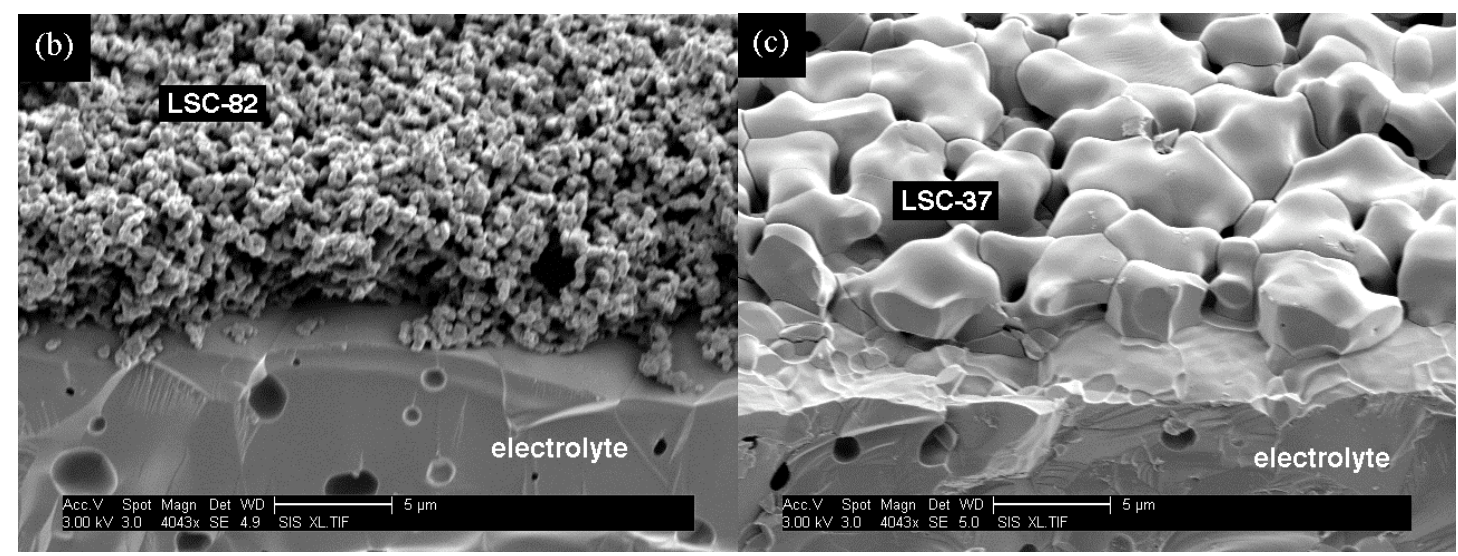

Fig. A-4. SEM micrographs of LSC electrodes examined in this study.

The microstructures of the electrodes were examined using SEM, with details presented elsewhere ${ }^{[7]}$. As shown in Fig 4, the LSC-82 electrode exhibits very porous microstructure with high surface area, while LSC-37 shows an over-sintered, low surface area microstructure consisting of grains with typical size of $5 \mu \mathrm{m}$, packed together or connected by necks. This lessthan-ideal morphology for LSC-37 was deliberate (using a high firing temperature of $1250^{\circ} \mathrm{C}$ ), in order to achieve a very different characteristic frequency than LSC-82, while maintaining a 
somewhat similar characteristic resistance. While we might expect LSC-37 to have a higher general performance than LSC-82 due to its higher bulk ionic transport, the disadvantageous microstructure of the LSC-37 electrode is expected to at least partially balance its performance relative to the LSC-82 electrode. In contrast, the LSC-37 electrode is expected to have a much higher chemical capacitance, and thus a much lower response frequency ${ }^{[9]}$.

The active WE area of each microelectrode cell was estimated from SEM images. For pattern A, the top view of the WE was examined; for pattern B - the cross section of the fractured cell was studied post-mortem. In addition, by measuring the ohmic portion of the impedance of the half-cell using impedance, we could calculate the apparent WE width using equation A-1, providing an additional (independent) measure of the size of the contact area of the WE.

In the case of pattern A, viscosity of the electrode ink strongly influences the final active area of the WE strips. However, in the case of Pt, the electrode ink was sufficiently viscous that the width of WE was close to the designed width of $150 \mu \mathrm{m}$. As for pattern $\mathrm{B}$, the width of the perovskite electrodes was significantly less than designed for reasons described below; observations ranged anywhere from 50 100 microns. As described below, estimates of the electrode width from impedance (based on eqn A-1) varied from 40 100 microns.

In most cases one geometrically symmetric cell and two half cells (with electrodes identical to those on the geometrically symmetric cell) were installed on a single test-stand having room for three cells. This helped to insure identical testing conditions, important later for relating the performance of these cells to each other. For connection of the cells with the external circuit, silver wires were attached to the electrodes or to the electrode contact areas (in half-cells) using silver paste (see photos in Fig. A-2(d)). The stand with the cells was installed in a vertical test stand similar that described elsewhere ${ }^{[10]}$.

Measurements of a.c. and d.c. polarization were made using a Solartron SI 1287 potentiostat and 1252A frequency response analyzer. A.c. impedance was measured at zero bias in the frequency range of $0.1 \mathrm{~Hz}-300 \mathrm{kHz}$. The measurements were done at $750^{\circ} \mathrm{C}$ in air, as well as in a blended gas of $1 \% \mathrm{O}_{2}$ in $\mathrm{N}_{2}$. After changing the temperature or atmosphere, the cells were kept under constant $T$ and $P_{\mathrm{O} 2}$ conditions until they showed repeatable impedance data within a time window of several hours. Extending our measurements into the nonlinear realm, the d.c. polarization behavior of $\mathrm{Pt}(\mathrm{A}), \mathrm{Pt}(\mathrm{sym}), \mathrm{LSC}-82(\mathrm{~B})$ and LSC-82(sym) cells were studied at $750^{\circ} \mathrm{C}$ in air and nitrogen/oxygen gas mixture containing $1 \%$ of oxygen. All polarization measurements were performed in potentiodynamic mode from 0 to $\pm 0.5 \mathrm{~V}$ for the half-cells, and from 0 to $\pm 1 \mathrm{~V}$ for the symmetric cells. Cyclic voltammagrams were measured in potentiodynamic mode with the scan rate $0.25 \mu \mathrm{V} / \mathrm{s}$ or $10 \mu \mathrm{V} / \mathrm{s}$. In the case of Pt electrodes, significant hysteresis was observed. In most cases, a second scan at the same sweep rate was enough to establish repeatability.

\section{A-5 Results and Discussion}

The conductivity of SDC, measured using the d.c. four-probe method, was found to be independent of whether was the sample had been laminated. These results together with SEM images of fracture cross-sections indicate that the laminated SDC plates were dense and uniform in this study. The measured value of conductivity was found to be $0.028 \pm 0.002 \mathrm{~S} / \mathrm{cm}$ at $750^{\circ} \mathrm{C}$, which is consistent with the data reported in reference ${ }^{[11]}$. 
The electrolyte conductivity was also estimated from a.c. impedance spectra for $\mathrm{Pt}(\mathrm{sym})$ and LSC-82(sym) cells (see Table A-1 for the configuration of these cells). The high frequency intersection of the impedance spectrum with the real impedance axis, $R_{\mathrm{HF}, \mathrm{sym}}$, was taken to be the electrolyte ohmic resistance. The ionic conductivity, $\sigma$, was then calculated as $\sigma=L / R_{H F, s y m}$, where $L$ is electrolyte thickness. The values of SDC conductivity calculated for the cells with different electrodes at different oxygen partial pressures exhibited little variation; they were equal to $0.024 \pm 0.001 \mathrm{~S} / \mathrm{cm}$ and $0.023 \pm 0.001 \mathrm{~S} / \mathrm{cm}$ for the cells with Pt and LSC-82 electrodes, respectively. This value is less than the conductivity of the electrolyte measured by d.c. fourprobe method by about $15 \%$. This discrepancy may be due to the contribution of contact resistance between electrode and current collector, as reported by Jiang et al. ${ }^{[12]}$. It may also represent anisotropy of the ionic conductivity, since the grain structure of the tape-cast layers may not be isotropic. In subsequent analysis we chose to use values based on the cell impedance $(0.023 \pm 0.001 \mathrm{~S} / \mathrm{cm})$; however this has little influence on our results.

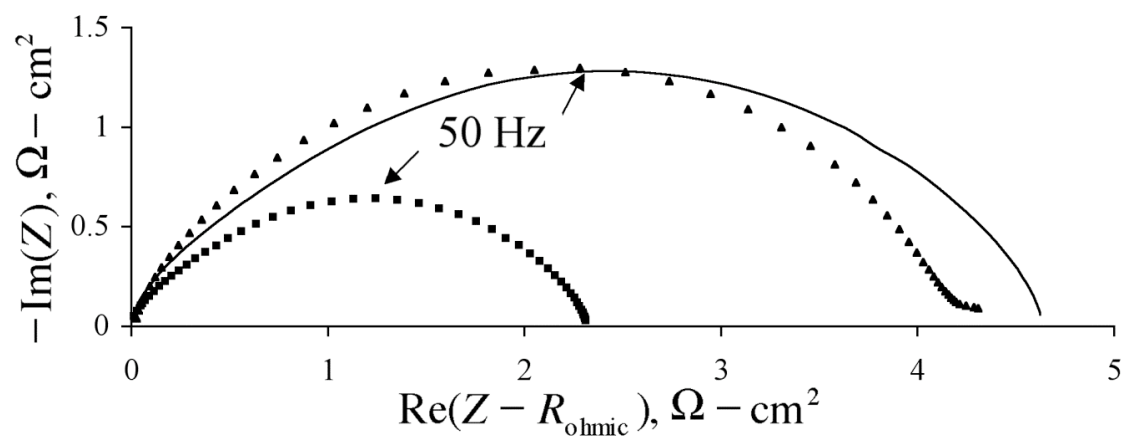

Fig. A-5. Impedances of a Pt(A) half-cell (squares) and a Pt(sym) symmetric cell (triangles) at $750^{\circ} \mathrm{C}$ in air. Solid line: the half-cell data multiplied by 2 .

The area-specific impedance plots for the $\mathrm{Pt}(\mathrm{sym})$ and $\mathrm{Pt}(\mathrm{A})$ cells in air at $750^{\circ} \mathrm{C}$ are shown in Fig. A-5. The high frequency intercept of the Nyquist plots with the real impedance axis, which is considered as the electrolyte ohmic resistance, is subtracted from these data. In the case of the $\mathrm{Pt}(\mathrm{A})$ cell, the active area was assumed to be the total exposed geometric area of the WE strips, which agreed well with that calculated using eqn. A-1, based on the ohmic intercept of the impedance. The spectra consist of one depressed semicircle with an apex frequency of 50 $\mathrm{Hz}$ for both cells.

Multiplication of the half-cell response of the $\mathrm{Pt}(\mathrm{A})$ cell by two is expected to match the semicircle for the symmetric cell. As shown in Fig. A-5, there is a good agreement between the measured impedance spectrum for symmetric cell and the doubled arc of the half-cell. The difference in the electrode resistance of about $10 \%$ can be attributed to fabrication variability, error of the working electrode area estimation (especially in the half-cell), or to slightly nonuniform current distribution over the electrode strip. 
Since the symmetric and half-cell impedances compared above have the same response frequency, one can only evaluate them on the basis of relative magnitude. A more stringent test of the microelectrode half-cell is to consider two microelectrode cells with working electrodes exhibiting different electrochemical behavior. In order to implement this test, cells LSC-82(B) and LSC-37(B) (definitions in Table A-1) were tested, and their responses compared to that of cell 37/SDC/82.

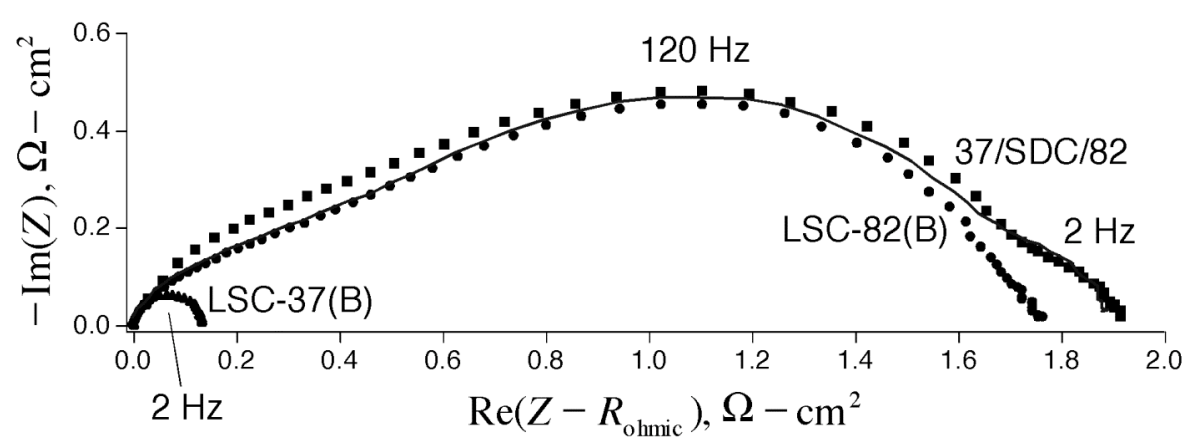

Fig. A-6. Impedances of a LSC37(B) half-cell (triangles), LSC82(B) half-cell (circles), and a $37 / \mathrm{SDC} / 82$ cell (squares). Solid line: sum of the two half-cell impedances.

The area-specific impedance spectra for LSC-82(B) and LSC-37(B) cells are shown in Fig. A-6. The measurements were made at $750^{\circ} \mathrm{C}$ in air. High frequency ohmic resistance is subtracted from these plots. The area of the pattern B cells in this case were calculated based on SEM observations of the WE width ( 100 microns). For LSC-82(B) this value agrees reasonably well with the WE width calculated using eqn. A-1 (85 microns). Unfortunately it was not possible to confirm the width of the LSC-37(B) WE based on eqn A-1 due to uncertainty in the ohmic intercept (obscured by inductive behavior at high frequency).

Fig. A-6 shows that the impedance spectrum for LSC-37(B) consists of one semicircle with characteristic frequency of about $2 \mathrm{~Hz}$. The corresponding capacitance is estimated to be $\sim 0.5 \mathrm{~F} / \mathrm{cm}^{2}$. This large value of capacitance has been observed for dense LSC electrodes with high levels of Sr doping, and is generally associated with the change of oxygen content in the oxide electrode ${ }^{[9,13]}$. The impedance of the LSC-82 electrode is more complex. It consists of at least two arcs: a high frequency arc (several $\mathrm{kHz}$ ), and a second arc with characteristic frequency of about $120 \mathrm{~Hz}$. The capacitance of the high frequency response has the order of $10^{-4} \mathrm{~F} / \mathrm{cm}^{2}$, which is typical for charge transfer at the electrode/electrolyte interface. The low frequency arc may be related to chemical processes such as oxygen surface exchange, solid-state and/or surface diffusion, and gas diffusion inside and outside of the porous electrode ${ }^{[9,10]}$.

Adding together the impedance data for LSC-37 and LSC-82 electrodes we obtain a calculated impedance which is expected to match the measured impedance of a geometrically symmetric cell consisting of one LSC-37 electrode and one LSC-82 electrode. This calculated response is compared to the actual measured response of cell 82/SDC/37 in Fig. A-6. Agreement is good over the range of frequencies studied. In addition, frequency response characteristics of LSC-82 (counterelectrode in the LSC-37(B) cell) are noticeably absent from the LSC-37(B) half- 
cell response. These results suggests that the microelectrode half-cells provide good frequency isolation, and can be quantitative in terms of frequency analysis of individual electrode response.

Extending our evaluation into the nonlinear realm, the current-overpotential characteristics of LSC-82/LSC were evaluated based on anodic and cathodic potentiodynamic measurements on a LSC-82(B) cell. For the particular cell studied, the overlap of the two mask layers was significant, leading to an open WE width of only $\sim 50$ microns (as determined from SEM post-mortem analysis). This value agrees well with that calculated using eqn. A-1 (50 microns) based on the ohmic resistance of the cell $\left(R_{\mathrm{E}}=0.95 \Omega-\mathrm{cm}^{2}\right.$, obtained from the highfrequency intercept of the impedance). Thus the value of $2 a=50$ microns was used to calculate the average current density in the potentiodynamic measurements. The anodic and cathodic overpotentials ( $\eta_{\text {anodic }}$ and $\eta_{\text {cathodic }}$ ) were then calculated by subtracting the ohmic contribution of the electrolyte:

$$
\eta_{W E}(i)=\left(V_{W E}-V_{R E}\right)-U_{E} .
$$

In order to be able to compare anodic and cathodic polarization at the same values of current density, the potentiodynamic data were fit to a 7-term polynomial, providing an interpolation function for $\eta_{\text {anodic }}(i)$ and $\eta_{\text {cathodic }}(i)$.
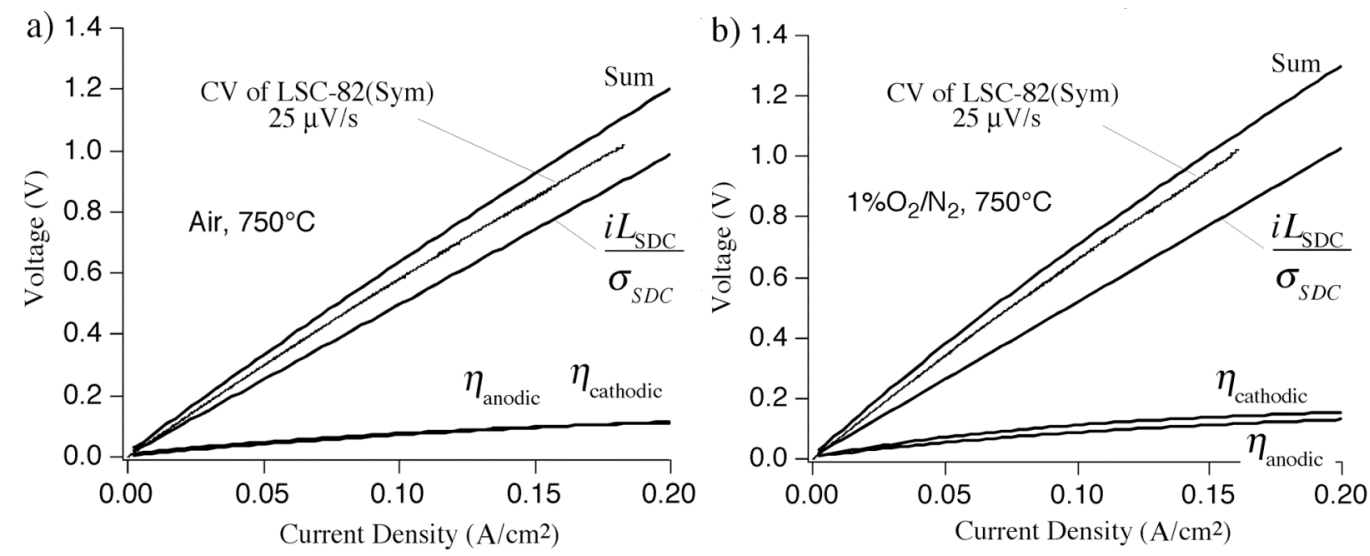

Fig. A-7. Electrode polarization behavior of LSC-82/SDC, based on the measured steadystate polarization of a LSC-82(B) half-cell at $750^{\circ} \mathrm{C}$ and at a) $P_{\mathrm{O} 2}=0.21$ and b) $P_{\mathrm{O} 2}=0.21$. $\eta_{\mathrm{a}}$ : anode overpotential; $\eta_{\mathrm{c}}$ : cathode overpotential; $i L / \sigma$ : measured ohmic resistance of a symmetric LSC-82/SDC/LSC-82 cell; Sum: prediction of the polarization behavior of a symmetric LSC-82/SDC/LSC-82 cell based on the sum of $\eta_{\mathrm{a}}+\eta_{\mathrm{c}}+i L / \sigma$. Thin line: measured polarization of an actual symmetric LSC-82/SDC/LSC-82 cell.

Fig. A-7 summarizes these calculations for anodic and cathodic polarization of LSC-82/SDC at $750^{\circ} \mathrm{C}$ in air and in $1 \% \mathrm{O}_{2} / \mathrm{N}_{2}$. The polarization behavior is highly symmetric, with anodic and cathodic over potentials ( $\eta_{\text {anodic }}$ and $\eta_{\text {cathodic }}$ ) lying virtually on top of one another in air. This is consistent with the high degree of linearity of LSC electrodes at these relatively low current densities ${ }^{[9]}$. As expected, anodic and cathodic overpotential increase with decreasing $P_{\mathrm{O} 2}$, becoming somewhat less linear, and the cathode polarization becomes slightly larger than the 
anodic anodic polarization at the same current density, possibly indicating the onset of gas-phase mass-transfer effects ${ }^{[10]}$.

Assuming these overpotentials are accurate, one should be able to predict the polarization behavior of a symmetric cell (LSC-82(Sym)), having the same electrodes as LSC-82(B). This was accomplished by first measuring the ohmic resistance $\left(L_{\mathrm{SDC}} / \sigma_{\mathrm{SDC}}\right)$ of LSC-82(Sym) using a.c. impedance. The polarization behavior of LSC-82(Sym) was then predicted over a range of current densities as:

$$
V_{\mathrm{Sum}}=i \frac{L_{\mathrm{SDC}}}{\sigma_{\mathrm{SDC}}}+\eta_{\text {anodic }}(i)+\eta_{\text {cathodic }}(i)
$$

Fig. A-7 compares the calculated sum in eqn. A-3 with cyclic voltammagrams of LSC$82(\mathrm{Sym})$ at $750^{\circ} \mathrm{C}$ in air and $1 \% \mathrm{O}_{2}$. Agreement is excellent, given our uncertainties in the exact electrode contact geometry, and inherent variability in fabrication among electrode samples.

\section{A-6 Summary}

Microelectrode half-cells comprising porous $\mathrm{La}_{1-\mathrm{x}} \mathrm{Sr}_{\mathrm{x}} \mathrm{CoO}_{3-\delta}$ (LSC) cathodes on dense samaria doped ceria (SDC) were fabricated using typical materials and techniques used in SOFC manufacturing. The a.c. and d.c. polarization behavior of these cells were measured, and the results evaluated quantitatively in terms of ohmic resistance, electrode overpotential, and frequency response. The results suggest that microelectrodes offer a promising alternative to other types of half-cells for isolating the frequency characteristics and overpotential of SOFC electrodes.

Two different microelectrode patters (pattern A and pattern B) were considered. We found that pattern $\mathrm{B}$ is generally superior for metal-oxide electrodes, since it does not require the use of a current collector. However, pattern-B requires an insulating mask material with an areaspecific resistance $>25 \mathrm{~kW}-\mathrm{cm}^{2}$, which we found difficult to process as a single thin layer. Our current state-of-the art mask material (physical mixture of $\mathrm{MgO}$ and spinel, processed in two separate coats) is generally too thick ( $>10$ microns), and difficult to pattern. A maturing of the materials and processing of the mask material is needed before the techniques outlined in this paper will be fully quantitative. However, as will be shown in Section D, a major benefit of the microelectrode half-cells is the ability to simultaneously test numerous identical cells in parallel, increasing experimental throughput. 


\section{B. Nonlinear Impedance Spectroscopy (NLEIS)}

\section{B-1 Introduction}

Electrochemical Impedance Spectroscopy (EIS) is used extensively to probe the electrochemical characteristics of solid oxide fuel cell (SOFC) electrodes. While EIS has proven to be a powerful technique for isolating and characterizing electrode response, detailed analysis of EIS data provides only limited insight regarding the specific rate-determining steps governing electrode reaction mechanisms. A major factor limiting the usefulness of EIS data is overlap or dispersion in the frequency domain among physical processes governing electrode reactions, making them difficult to resolve entirely by timescale. Another factor is that different mechanistic models for a given reaction often predict very similar impedance response after the governing equations have been linearized.

In order to gain further insight into the reaction and degradation mechanisms governing SOFC cathodes, we have extended EIS to probe both linear and nonlinear responses. This technique, which has been called Nonlinear EIS (NLEIS) ${ }^{[14]}$, involves the measurement of nonlinear $2^{\text {nd }}$ and higher order voltage harmonics resulting from moderate amplitude current perturbations. By the excitation of nonlinear responses and interactions, NLEIS potentially offers improved identification of physical steps via nonlinearity and extent of coupling, as well as timescale. The benefit of measuring nonlinear response has been demonstrated in a variety of fields including hydrodynamics ${ }^{[15-17]}$, corrosion ${ }^{[14,18]}$, nonlinear optics ${ }^{[19,20]}$, and electro analytical chemistry $^{[21,22]}$. By combining this approach with well-defined materials and microstructures, it may become possible to identify the relevant mechanisms (or at least narrow the list of possibilities).

\section{B-2. NLEIS Apparatus and Procedures}

A schematic of the NLEIS apparatus is illustrated in Fig. B-1. As with traditional EIS, the cell of interest is connected to a potentiostat, which regulates and measures voltage and current. For NLEIS measurements, the traditional frequency response analyzer is replaced with a Dell PC containing National Instruments (NI) signal generator and A/D acquisition cards. The signal generator is connected to the POL I/P (set-point) coaxial input of the potentiostat, while the VOUT and IOUT coaxial outputs of the potentiostat were connected to the digitizers. By varying the frequency and amplitude of the generated signal, the resulting voltage response and the current modulation itself can be measured and recorded synchronously. A computer running Labview is used to control the potentiostat, as well as a sinusoidal function generator and two synchronized high-speed digitizers.

Two instruments were developed under sponsorship of this program. The first is based on a NI-5401 function generator linked synchronously to two NI-5911 high-speed digitizers. The second is based on a NI-PCI-5412 Waveform Generator and a dual-channel NI-PCI-5122 Digitizer. The second of these two instruments has considerably better signal-to-noise characteristics, and was used for most of the measurements reported in part B. 


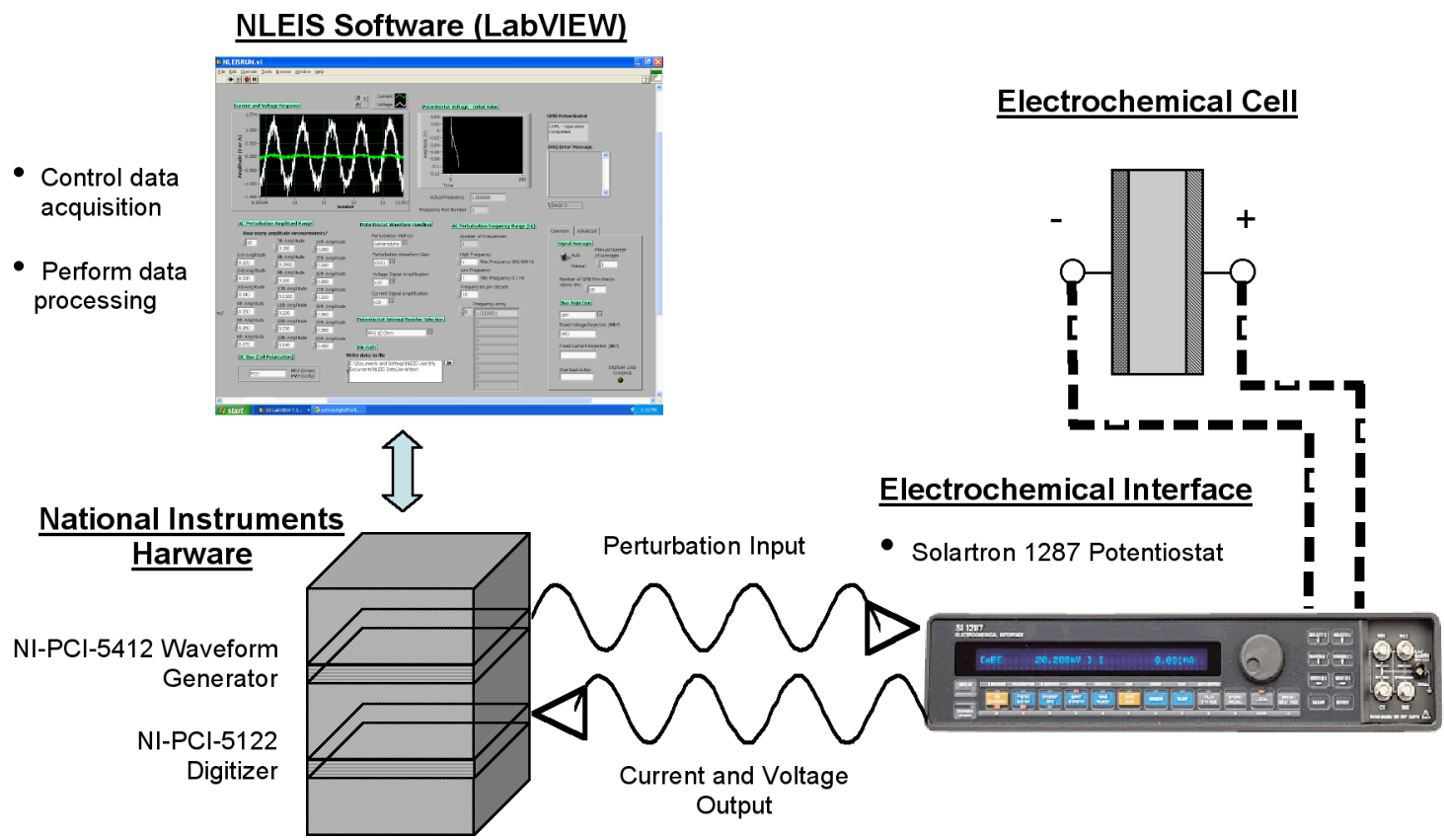

Figure B-1: Schematic of the apparatus used to conduct NLEIS measurements.

\section{B-3. Signal Acquisition and Analysis}

For each amplitude and frequency measurement, the system is modulated for at least three cycles prior to data acquisition to ensure steady periodic behavior. A total of 4096 data points are then collected at an acquisition rate of $40.96 \tilde{\omega} / 2 \pi$, where $\tilde{\omega}$ is the radial frequency of the current perturbation, providing 100 cycles of current and voltage modulation. Fig. B-2 shows examples of time dependent data for porous LSC electrodes on SDC (prepared as described in Section D).

In order to define the line width of the subsequent frequency-domain data, the time domain data are multiplied by a Gaussian apodization window $W(t)$ :

$$
W(t)=\exp \left(-\left(\frac{\tilde{\omega} t}{2 \pi b}\right)^{2}\right)
$$

As shown in Figs. B-2(c,d), apodization transforms the steady periodic time-domain data (of undefined duration) into a time-decaying signal. The application of this transformation results in a frequency-domain signal having a well-defined Gaussian line width proportional to $b$ cycles, and a defined quantity of points, that is controlled by the acquisition rate. For the experiments reported below, an apodization window of $b=7.5$ cycles was used. This value provides an adequate number of data points defining the frequency-domain signal, while not being so broad as to lose signal resolution ${ }^{[23]}$. 

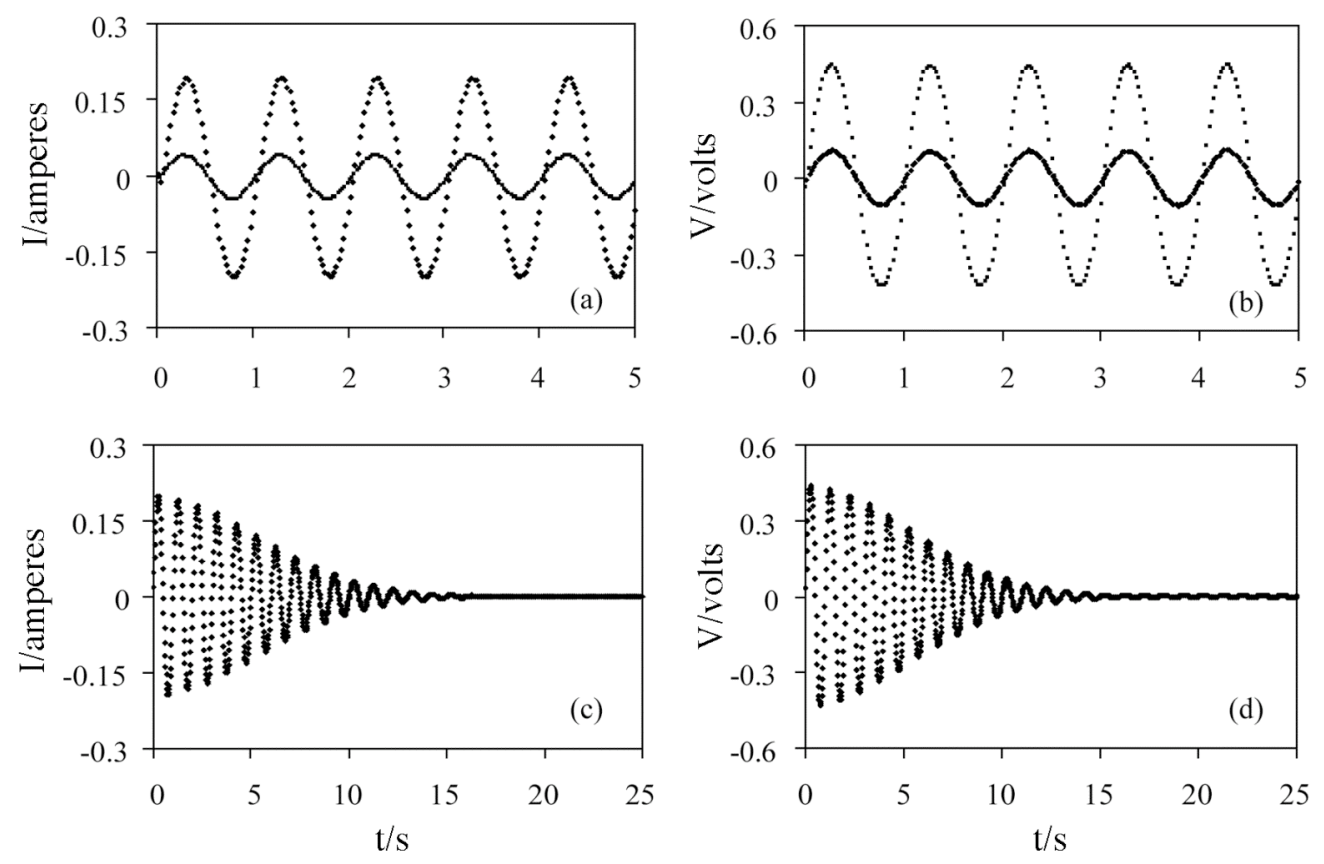

Figure B-2: Measured current perturbation, and potential response, applied to a LSC$82 / \mathrm{SDC} / \mathrm{LSC}-82$ cell at $750^{\circ} \mathrm{C}$ in air, at a frequency of 1.0 cycles/second (Hz) a) Raw data showing current as a function of time at perturbation amplitudes of $30 \mathrm{~mA}$ and 200 $\mathrm{mA}$. b) Corresponding potential response for the perturbation amplitudes in (a). c) Plot of the Gaussian-apodized current data at an amplitude of $200 \mathrm{~mA}$. d) Plot of the Gaussianapodized potential response at a current perturbation amplitude of $200 \mathrm{~mA}$. Graphs (a) and (b) display only 5 cycles to better illustrate the shape of the sinusoids.

Following apodization, the time-domain data are converted to the frequency domain using the complex fast Fourier-transform (FFT) algorithm. Fig. B-3 shows real and imaginary parts of the Fourier-transformed current and potential for the data in Fig B-2. The Fourier-transformed current has a harmonic signal only at the input frequency, reflecting the fact that the input is a purely sinusoidal waveform. In contrast, the Fourier-transformed potential has a signal at the input frequency as well as integer multiples of the input frequency, indicating that the potential response is nonlinear. The peak at the excitation frequency represents the first order (linear) harmonic of the potential. The peak at $k$ times the excitation frequency corresponds to the $k^{\text {th }}$ harmonic. Comparing signals at 30 and $200 \mathrm{~mA} / \mathrm{cm}^{2}$, these results show that as the amplitude of the input signal increases, the higher order harmonics become more clearly discernable, growing nonlinearly out of the background noise.

For a symmetric cell with identical electrodes, harmonic signals of the potential response were found to occur only at odd-numbered harmonics $\left(1^{\text {st }}, 3^{\text {rd }}, 5^{\text {th }}\right.$, etc.). This observation can be attributed to the physical inversion-symmetry of the symmetric cell. In a physically symmetric system, the response is purely anti-symmetric, corresponding to superposition of odd harmonics generated by the two electrodes, and nullification of even harmonics $\left(2^{\text {nd }}, 4^{\text {th }}, 6^{\text {th }}\right.$, etc. $)$. 

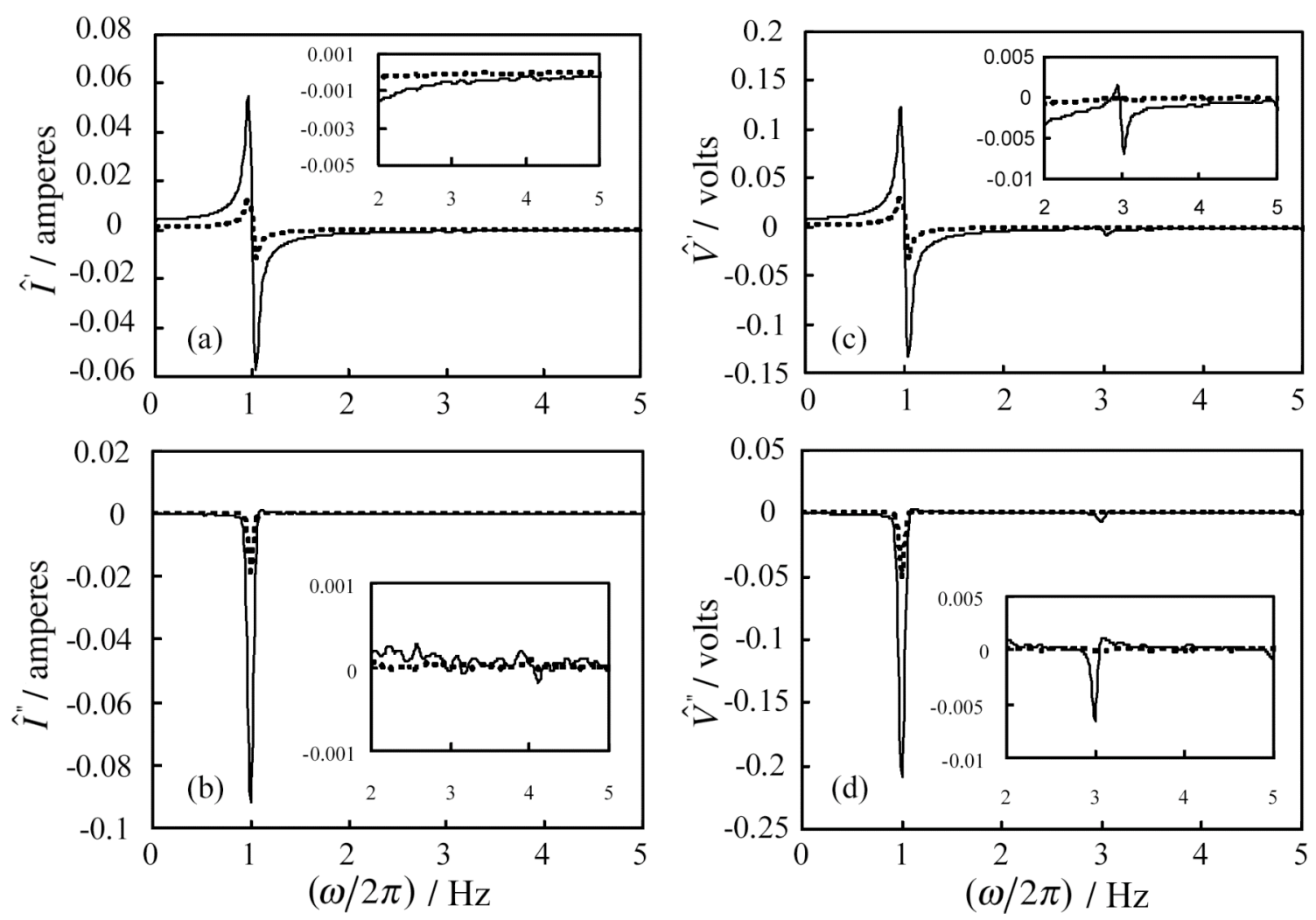

Figure B-3: Plots of the Fourier-transformed current and potential data at a perturbation frequency of $1.0 \mathrm{~Hz}$ and amplitudes of $30 \mathrm{~mA}(---)$, and $200 \mathrm{~mA}(-)$. Inset: plots at expanded scale near the higher-order harmonics. a) Real current; $b$ ) Imaginary current; c) Real potential; d) Imaginary potential.
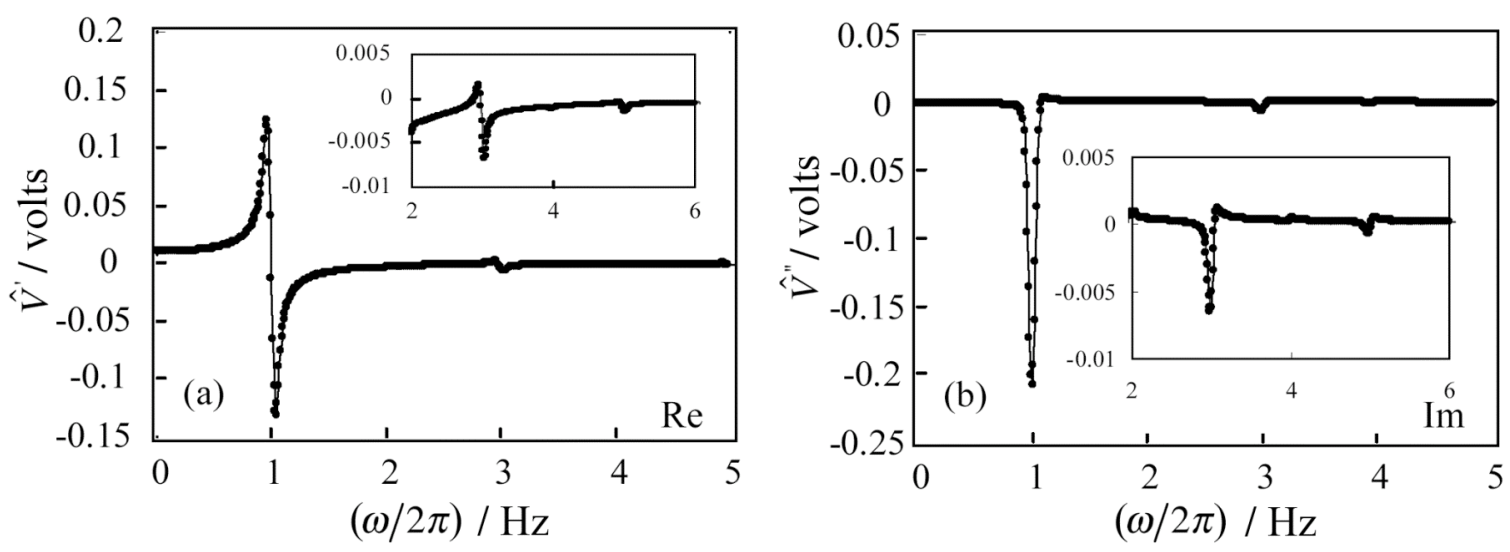

Figure B-4: Plots of the Fourier-transformed potential for a current perturbation of $1.0 \mathrm{~Hz}$ and $160 \mathrm{~mA}$. Raw data (symbols) is shown with least squares fit data (line). 
Although the harmonics can be readily observed in the frequency-domain data, lower-order harmonics often create a baseline distortion for the (usually much smaller) higher-order harmonics, making it inconvenient to quantify each harmonic individually. To circumvent this problem, the time domain signal are expressed as a linear superposition of steady-periodic harmonics of unknown amplitude and phase:

$$
V(\tilde{i}, \tilde{\omega}, t)=\frac{1}{2} \sum_{k=0}^{\infty} \hat{V}_{k}(\tilde{i}, \tilde{\omega}) \exp (j k \tilde{\omega} t)+\hat{V}_{-k}(\tilde{i}, \tilde{\omega}) \exp (-j k \tilde{\omega} t)
$$

where $\tilde{\omega}$ and $\tilde{i}$ are the frequency and amplitude of the frequency perturbation, and $\hat{V}_{ \pm k}=\hat{V}_{k}^{\prime} \pm j \hat{V}_{k}^{\prime \prime}$ are complex Fourier coefficients corresponding to each harmonic (the term $j$ denoting $\sqrt{-1}$ ). A prime denotes the real part of the Fourier coefficient, and a double prime represents the imaginary part.

Time-apodization and Fourier-transformation of eqn. B-2a yields:

$$
\begin{aligned}
\hat{V}(\tilde{i}, \tilde{\omega}, \omega)= & \frac{1}{2} \sum_{k=0}^{\infty} \hat{V}_{k}^{\prime}\left(G_{k}(\omega)+G_{-k}(\omega)\right)+\hat{V}_{k}^{\prime \prime}\left(D_{k}(\omega)-D_{-k}(\omega)\right), \\
& +\frac{j}{2} \sum_{k=0}^{\infty} \hat{V}_{k}^{\prime \prime}\left(G_{k}(\omega)-G_{-k}(\omega)\right)+\hat{V}_{k}^{\prime}\left(-D_{k}(\omega)-D_{-k}(\omega)\right)
\end{aligned}
$$

where:

$$
\begin{aligned}
& G_{k}(\omega)=\frac{b \sqrt{\pi}}{\tilde{\omega}} \exp \left(\frac{-(\omega-k \tilde{\omega})^{2} \pi^{2} b^{2}}{\tilde{\omega}^{2}}\right) \\
& D_{k}(\omega)=\frac{b \sqrt{\pi}}{\tilde{\omega}} \exp \left(\frac{-(\omega-k \tilde{\omega})^{2} \pi^{2} b^{2}}{\tilde{\omega}^{2}}\right) \operatorname{erfi}\left(\frac{(\omega-k \tilde{\omega}) \pi b}{\tilde{\omega}}\right)
\end{aligned}
$$

where $\operatorname{erfi}(x)$ is the imaginary error function, related to the error function by $\operatorname{erfi}(x)=-j \cdot \operatorname{erf}(x)$. By fitting the real and imaginary parts of the frequency-domain data to eqn. B-2b using linear least squares, the real and imaginary part of each voltage harmonic (to the desired order in $k$ ) can be recovered simultaneously. Fig. B-4 shows an example of this procedure, to 5th order.

A similar procedure is used to fit the Fourier-transformed current data, allowing extraction of $\hat{I}_{1}=\tilde{i} \exp \left(j \varphi_{1}\right)$, where $\varphi_{1}$ is an arbitrary phase factor representing the point in the perturbation cycle where data acquisition was begun $(t=0)$. Since the voltage and current data are acquired synchronously, each voltage harmonic is multiplied by a 1 st-order phase correction $\exp \left(-j k \varphi_{1}\right)$ in order to establish its absolute phase relative to the current perturbation.

Because the system is nonlinear, the magnitude of each voltage harmonic $\hat{V}_{k}(\tilde{i}, \tilde{\omega})$ generally depends nonlinearly on the current perturbation amplitude. As shown in reference ${ }^{[15]}$ and ${ }^{[16]}$, this nonlinear dependence can be expressed as a power series in amplitude:

$$
\hat{V}_{k}(\tilde{i}, \tilde{\omega})=\sum_{r=1}^{\infty} \alpha^{k+2 r-2} \hat{V}_{k, k+2 r-2}(\tilde{\omega})
$$


where $\alpha=\tilde{i} / i^{*}$ is the current perturbation amplitude, nondimensionalized based on $i^{*}$, a real constant having units of current density that characterizes the onset of nonlinearity in the system (see reference ${ }^{[23]}$ for a physical interpretation of $i^{*}$ ).

As with the Fourier coefficients $\hat{V}_{k}(\tilde{i}, \tilde{\omega})$, each term in the power series $\hat{V}_{k, m}(\tilde{\omega})$ has both a real and imaginary components $\hat{V}_{k, m}=\hat{V}_{k, m}^{\prime}+j \hat{V}_{k, m}^{\prime \prime}$ embodying magnitude and phase. However, the individual harmonic terms $\hat{V}_{k, m}(\tilde{\omega})$ are amplitude independent, and thus have the advantage of being purely frequency-dependent functions (like impedance measured at low amplitude). The impedance, $Z(\tilde{\omega})=\hat{V}_{1,1}(\tilde{\omega}) / i *$ is (by definition) proportional to the first order contribution to the first harmonic.
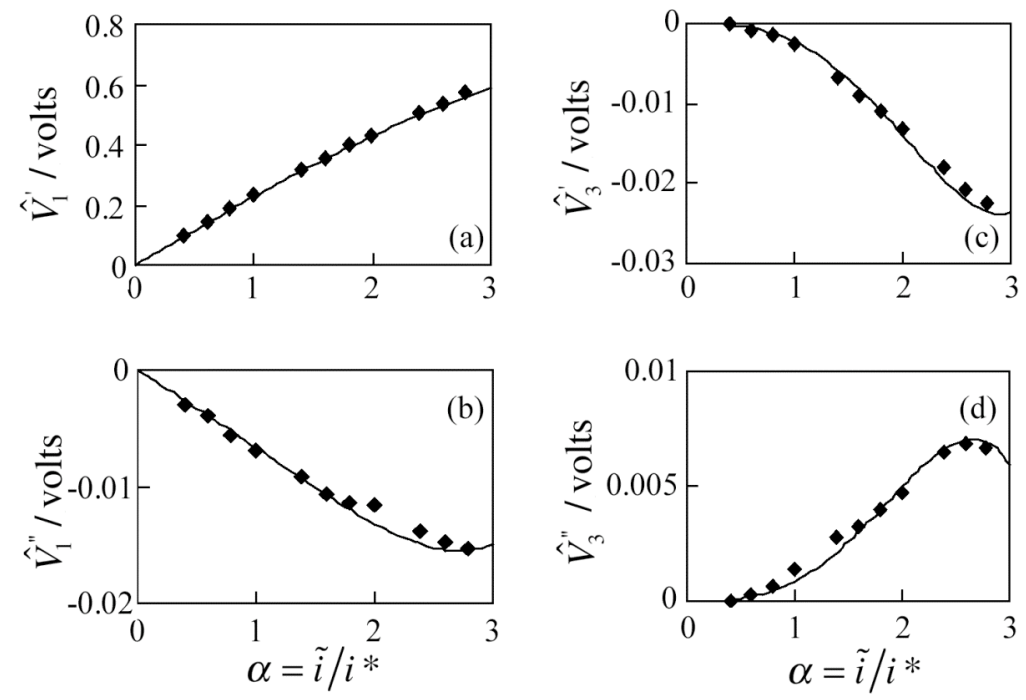

Figure B-5: Plots of the real and imaginary potential harmonics as a function of dimensionless current modulation amplitude, for a perturbation frequency of 1.0 Hz. The experimental results (symbols) are shown with the polynomial fit (line) to equations B-3a and $3 \mathrm{~b}$. a) Real $1^{\text {st }}$ (fundamental) harmonic; b) Imaginary $1^{\text {st }}$ harmonic; c) Real $3^{\text {rd }}$ harmonic; d) Imaginary $3^{\text {rd }}$ harmonic.

To determine the individual harmonic terms, $\hat{V}_{k, m}$, measurements are repeated at 10-15 amplitudes per frequency, and then each harmonic $\hat{V}_{k}$ is fit to a finite power series according to eqn. B-2c. One term beyond that desired is needed in the polynomial fit in order to absorb error due to higher order terms:

$$
\begin{aligned}
& \hat{V}_{1}(\tilde{i}, \tilde{\omega})=\alpha \hat{V}_{1,1}(\tilde{\omega})+\alpha^{3} \hat{V}_{1,3}(\tilde{\omega})+O\left[\alpha^{5}\right] \\
& \hat{V}_{2}(\tilde{i}, \tilde{\omega})=\alpha^{2} \hat{V}_{2.2}(\tilde{\omega})+\alpha^{4} \hat{V}_{2,4}(\tilde{\omega})+O\left[\alpha^{6}\right] \\
& \hat{V}_{3}(\tilde{i}, \tilde{\omega})=\alpha^{3} \hat{V}_{3,3}(\tilde{\omega})+\alpha^{5} \hat{V}_{3,5}(\tilde{\omega})+O\left[\alpha^{7}\right]
\end{aligned}
$$


Fig. B-5 shows the amplitude-dependence of the harmonics of the cell in Figs. B-2 $\sim$ B-4, and shows the corresponding polynomial fit. This procedure is repeated over the entire range of frequencies, resulting in amplitude-independent spectra as exemplified by the data in Fig. B-6 (where the data have been nondimensionalized as described in reference ${ }^{[23]}$ ). The interpretation of the higher harmonic spectra is discussed in the following sections.
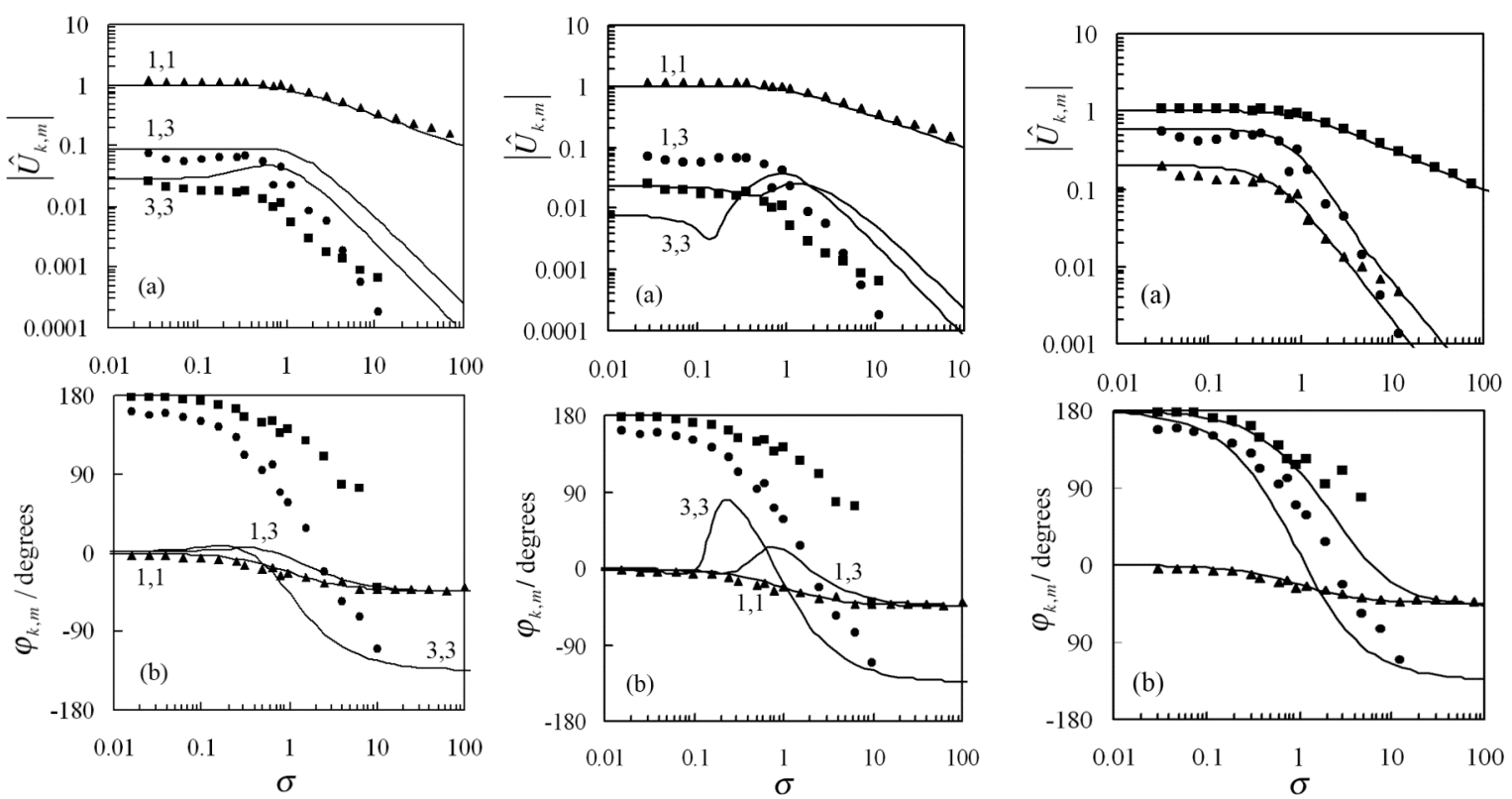

Fig. B-6. Bode plots of the measured (symbols) and calculated (lines) harmonic response functions $\hat{U}_{1,1}(\sigma)$ (triangles), $\hat{U}_{1,3}(\sigma)$ (squares), $\hat{U}_{3,3}(\sigma)$ (circles) of a symmetric porous $\mathrm{LSC} / \mathrm{GDC} / \mathrm{LSC}$ cell at $750^{\circ} \mathrm{C}$ in air. The left, middle and right sets of plots compare the same set of data to 3 different models for the $\mathrm{O}_{2}$ exchange kinetics. Only one set of kinetics comes close to explaining the higherharmonic data (right). Further details in Section D and reference ${ }^{[23]}$.

\section{B-4. Test-Bed Application \#1: Porous LSC on SDC.}

In order to determine the type of information obtainable from NLEIS measurements on porous SOFC cathodes, symmetrical cells consisting of two porous $\mathrm{La}_{0.8} \mathrm{Sr}_{0.2} \mathrm{CoO}_{3-\delta}$ (LSC-82) cathode layers were fabricated by screen-printing and firing LSC-82 ink onto pre-fired dense tape-cast 250 micron thick Sm-doped ceria (SDC) electrolyte. The green SDC tape $\left(79 \% \mathrm{CeO}_{2}\right.$, $20 \% \mathrm{Sm}_{2} \mathrm{O}_{3}, 1 \% \mathrm{TiO}_{2}$ as a sintering aid), made by Cerametec, Inc. (Salt Lake City), was cut into approximately $2.5 \mathrm{~cm} \times 2.5 \mathrm{~cm}$ square cells, and fired to $98+\%$ density at $1650^{\circ} \mathrm{C}$ for 4 hours in air. The LSC-82 ink was prepared from $\mathrm{La}_{1-\mathrm{x}} \mathrm{Sr}_{\mathrm{x}} \mathrm{CoO}_{3-\delta}$ powder of compositions $x=0.2$, obtained from Praxair Surface Technologies (Seattle). The ink was made using a 3-roll mill, blending the LSC-82 powder with alpha-terpineol, ethyl cellulose, ethanol, and oleic acid (all UHP grade from Alpha-Aesar). LSC electrodes of $1.0 \mathrm{~cm}^{2}$ area were then screen printed onto both sides of the dense electrolyte, and fired in air at $1150^{\circ} \mathrm{C}$ for 2 hours. Porous silver current collectors were screen printed onto the LSC electrodes and fired at $900^{\circ} \mathrm{C}$ for two hours. 

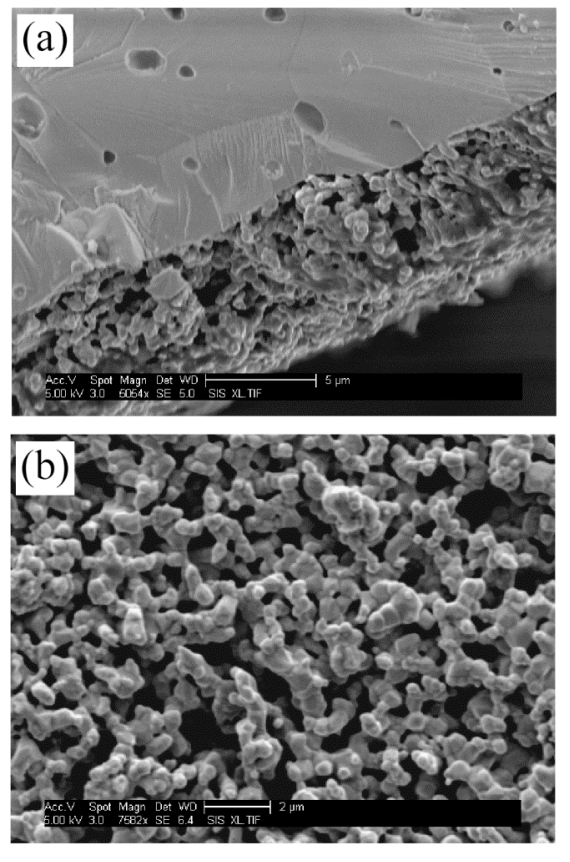

After sintering of the electrodes, the cell was examined with x-ray diffraction (Philips 1820XRD), and found to be single-phase with no X-ray-detectable impurities. Scanning electron microscopy SEM (FEI Sirion) was used to characterize the microstructure of the electrodes and verify the absence of minor second phases. Fig. B-7 shows an SEM image of fired $\mathrm{La}_{0.8} \mathrm{Sr}_{0.2} \mathrm{CoO}_{3-\delta}$ on ceria. The electrode thickness was determined from SEM analysis to be $\sim 6$ microns. Porosity and surface area of the electrodes were estimated based on the thickness, amount printed, and particle size in the SEM micrographs.

Figure B-7: SEM micrographs of the LSC82/SDC/LSC-82 cell, with electrodes sintered at $1150^{\circ} \mathrm{C}$. a) Fracture cross section showing the region near the LSC-82/SDC interface. b) Top surface of porous LSC-82.

NLEIS measurements were performed at $750^{\circ} \mathrm{C}$ in air at zero bias and frequencies between $0.1 \mathrm{~Hz}$ and 10,000 Hz. For each frequency, 10 perturbations of varying amplitude were applied. Data were processed as described above, and plotted as in Fig. B-6.

Figure B-6 shows that the first order harmonic spectrum (impedance) exhibits the same characteristic Gerischer response reported previously for LSC-82 on Sm-doped ceria ${ }^{[10]}$. This behavior has been explained previously using a model that assumes co-limitation by $\mathrm{O}_{2}$ reduction and bulk transport ${ }^{[10,24]}$. However, questions have remained about the uniqueness of this model vis-à-vis the true mechanism. In order to test this model further, we have extended it into the nonlinear realm, so as to also compare it to the higher harmonics ${ }^{[23]}$.

The complete details of the modeling are beyond the scope of this report, and can be found elsewhere ${ }^{[23]}$. However, a summary of the physical assumptions of this model include the following:

1. The mechanism involves co-limitation by kinetics and bulk transport.

2. Resistance to interfacial charge transfer at the LSC/electrolyte interface is negligible.

3. The active region of the electrode is significantly smaller than the electrode thickness, but large compared to microstructural features, and thus the model can be formulated in 1-D.

4. Gas phase diffusion has no effect on this system.

5. The mixed conductor has high electrical conductivity.

With these assumptions, the higher harmonic response of the electrode may be calculated for various scenarios involving the $\mathrm{O}_{2}$ exchange kinetics and thermodynamic properties of the material. A combination of computer software tools were used to obtain a solution to the relevant set of nonlinear equations ${ }^{[23]}$. Mathematica (Wolfram Research) was first used to derive (analytically) the harmonic equation coefficients entering the ordinary differential equations and 
boundary conditions. These equations were input into FEMLAB (Comsol AB), a finite element package for use with MATLAB (The Mathworks, Inc.).

Fig. B-6 compares the measured $\hat{U}_{1,1}(\sigma)$ spectrum (impedance) to that predicted by the model. Although the agreement between model and data is good, this result holds true regardless of the nonlinear kinetics chosen for the kinetics ${ }^{[23]}$. In other words, the impedance is entirely insensitive to the mechanism underlying $\mathrm{O}_{2}$ exchange.

In contrast, Fig. B-6 also compare the measured higher harmonic response $\left(\hat{U}_{3,3}(\sigma)\right.$ and $\left.\hat{U}_{1,3}(\sigma)\right)$ to three different nonlinear models for the kinetics ${ }^{[23]}$. It is clear that only the model on the far right comes close to explaining the third harmonic response. The model in this case is based on an empirical rate expression for $\mathrm{O}_{2}$ exchnage, and thus does not tell us about the mechanism directly. However, as discussed further in Section D, a more physical model can make use of the nonlinear information present in the harmonics to help decipher mechanism. The main point here is simply that the higher harmonics are measurable, and have additional nonlinear information about rates than the impedance alone.

\section{B-5. Test-Bed Application \#2: Mass Transfer Effects SOFC Tests.}

Pacific Northwest National Laboratory's (PNNL's) Materials Division is highly involved in solid oxide fuel cell (SOFC) development research. One particular area of focus is studying SOFC cathode performance and degradation (Dr. Steven Simner). A commonly used technique at PNNL for tracking performance vs. time is EIS. Since our group at UW is interested in EIS, and furthering SOFC development in general, we started a collaboration with PNNL having two goals: to assist PNNL with their EIS measurements and interpretation, and to assess the feasibility and potential of using NLEIS to study anode-supported SOFCs.
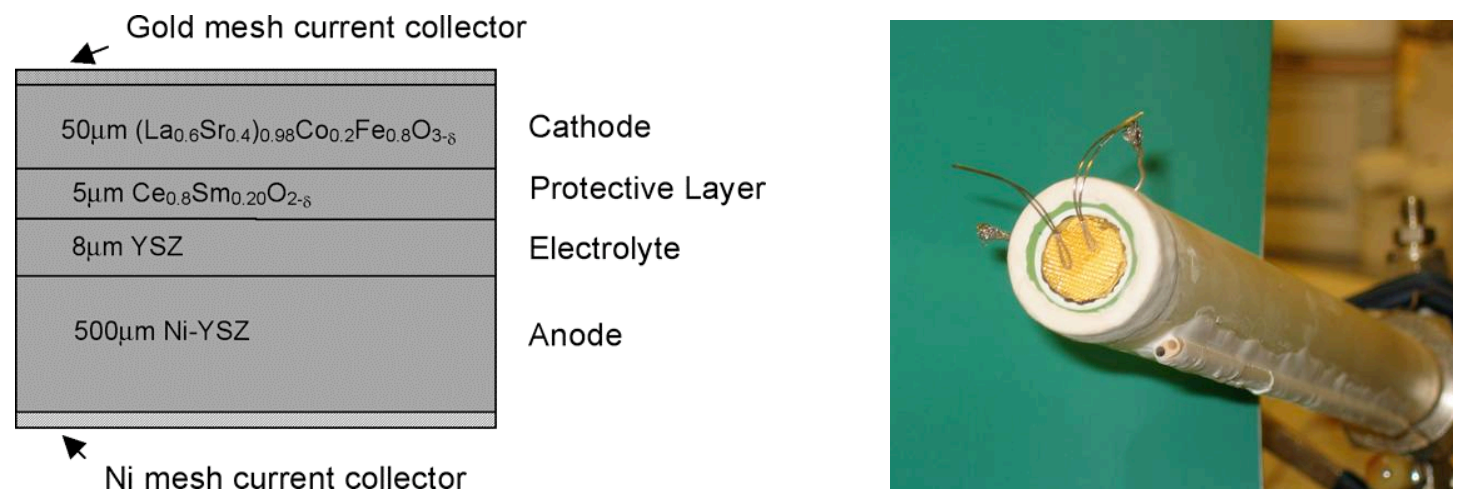

Fig. B-8. Left: Schematic diagram of the anode-supported SOFC button-cell fabricated by Simner et al. Right: Photograph of button cells with wire leads waiting to be tested.

Figure B-8 shows a schematic diagram of the anode-supported SOFC examined in this application. The cathode material in this case was $\left(\mathrm{La}_{0.6} \mathrm{Sr}_{0.4}\right)_{0.98} \mathrm{Co}_{0.2} \mathrm{Fe}_{0.8} \mathrm{O}_{3-\delta}$, a commonly investigated mixed conductor combining some of the advantages of LSC and LSF in durability and performance. The cells consisted of a $\sim 2.5 \mathrm{~cm}$ diameter, $\sim 500 \mu \mathrm{m}$ thick NiO-YSZ anode 
substrate composed of a bulk layer of $40 \% \mathrm{Ni}$ and $60 \%$ Yittria-stabilized zirconia (YSZ) with approximately $30 \%$ porosity, and a $5-10 \mu \mathrm{m}$ thick surface layer of $50 \% \mathrm{Ni}$ and $50 \% \mathrm{YSZ}$ (by volume). The electrolyte layer was $8 \mu \mathrm{m}$ of pure YSZ. A very thin layer of samaria-doped cerial (SDC) of composition $\mathrm{Ce}_{0.8} \mathrm{Sm}_{0.20} \mathrm{O}_{2-\delta}$ was used as a protective layer between the LSCF cathode and the YSZ electrolyte to suppress zirconate formation. The diameter of the cathode was $1.6 \mathrm{~cm}$. Details of the fabrication of the button-sized cells used in this study can be found in reference ${ }^{[25]}$. One benefit of collaborating with PNNL is the ability to work with materials having extremely reproducible materials preparation, tape-casting, tape-laminating and thin-film screen printing fabrication procedures ${ }^{[26]}$.

The button cells were mounted onto an alumina oxide tube (Fig. B-8, right), and placed within a 2-compartment test stand (Fig. B-12, left). This allowed the cells to be placed into a furnace for high-temperature testing, and also provided for gas-delivery capabilities to the anode and cathode sides of the cell. A variable mixture of hydrogen, nitrogen and $3 \mathrm{~mol} \%$ water were fed to the inner-tube and vented through the outter-tube via mass-flow controllers. Air or pure oxygen was blown onto the surface of the cathode. The cathode was not enclosed in a chamber like the anode; it was exposed to the atmosphere inside of the furnace. Typical total gas flow ranged from 100 SCCM to 300 SCCM, for the anode and cathode sides of the cell. Both sides were operated at $1 \mathrm{~atm}$ pressure.

One significant challenge associated with using EIS as a metric for this SOFC system is the lack of a reference electrode, which is difficult to incorporate in the thin cell design ${ }^{[26]}$. The lack of reference electrode prevents partitioning of the anode and cathode contributions to the overall impedance via half-cell measurements. So in order to separate the anode and cathode contributions in the EIS data, Simner independently manipulated the anode and cathode gas flows.

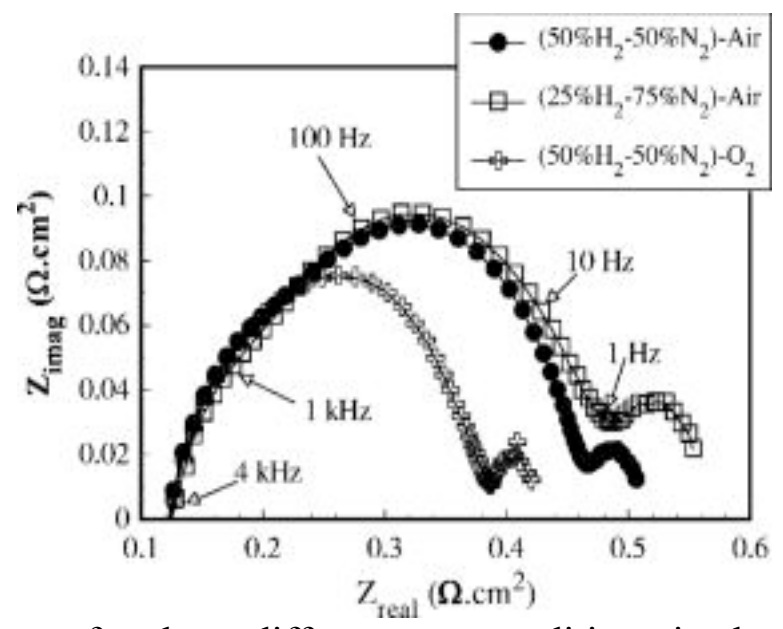

Figure B-9. EIS arcs for three different gas conditions in the SOFC test stand. The values in parentheses represent anode gas conditions. Total flow rates were 200 SCCM for both the anode and cathode. Data from reference ${ }^{[26]}$

Fig. B-9 shows typical EIS response under polarized conditions, where the anode and/or cathode gas conditions are varied. Simner identified three different arcs in this data: low $(<1 \mathrm{~Hz})$, medium $(1-500 \mathrm{~Hz})$, and high $(>1 \mathrm{~Hz})$. He concluded that the high frequency arc was influenced 
by oxygen concentration in the cathode feed gas, and thus must be related to processes occurring at the cathode. He also concluded that the low frequency arc was affected by changes in the hydrogen concentration in the gas fed to the anode, and thus must be related to processes on the anode side of the cell. These results were used as a general rule to separate the anode and cathode contributions to all EIS results.

However, it is well known that gas phase mass transfer can be manifest as impedance features that have nothing to do with electrode kinetics. To determine if such effects might be an issue here, we first measured $i-V$ characteristics as a function of polarization. Fig. B-10 shows the cell response, as a function of gas-phase feed conditions to the anode, starting with the nominal case of 100 SCCM hydrogen (H2) and 100 SCCM nitrogen (N2), and independently changing hydrogen concentration and molar flux. The $i-V$ plot shows the limiting current decreases for independent changes in molar hydrogen flow rate as well as $\mathrm{H}_{2}$ concentration.

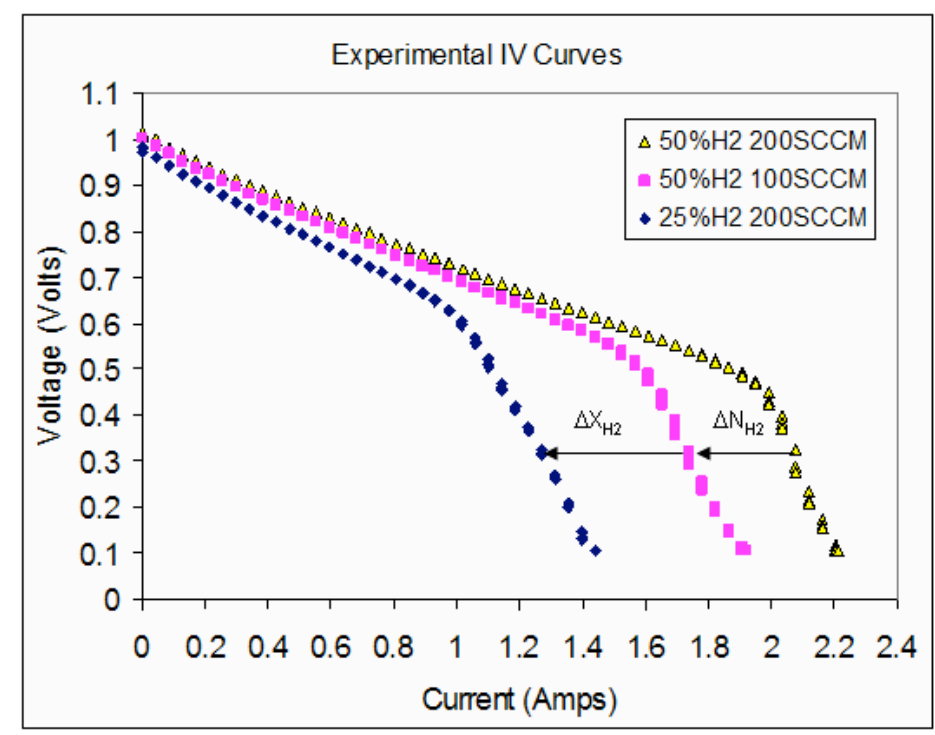

Figure B-10. $i-V$ characteristics of a PNNL button-cell at $750{ }^{\circ} \mathrm{C}, 1$ atm under various anode gas conditions (altering composition and flow rate independently).

\begin{tabular}{|c|c|c|}
\hline Gas Condition & $\begin{array}{c}\text { Current at } 0.1 \mathrm{~V} \\
\text { (Amps) }\end{array}$ & Utilization (\%) \\
\hline $50 \%$ H2 200 SCCM & 2.2 & 16.7 \\
\hline $50 \%$ H2 100 SCCM & 1.9 & 28.8 \\
\hline $25 \%$ H2 200 SCCM & 1.4 & 21.2 \\
\hline
\end{tabular}

Table B-1. Estimated hydrogen utilization at limiting current vs. gas condition at $750{ }^{\circ} \mathrm{C}, 1 \mathrm{~atm}$ total pressure. 
Table B-1 shows the estimated anode utilization under these conditions. These calculations show that the limiting current cannot be explained by reduced $\mathrm{H}_{2}$ concentration in a well-mixed anode chamber, rather there must be some kind of diffusion limitation. The observation that limiting current changes with $\mathrm{H}_{2}$ concentration as well as flow rate suggests that a stagnant gas layer above the cell may be at work. The fact that the limiting current comes on gradually rather than suddenly may indicate that water escape (rather than $\mathrm{H}_{2}$ supply) may be more critical.

The next step in this study was to perform EIS measurements. If a hydrogen gas diffusion limitation existed at the anode, it would be pronounced at higher bias where a larger hydrogen concentration gradient would exist between the bulk feed gas and the anode surface. EIS sweeps were taken for each gas condition in two different operating regimes: low current bias (in the kinetic region of the IV curve), and high current bias (in the mass-transfer limited regime). Figure B-11 shows one result from these experiments, using an AC amplitude of $250 \mathrm{~mA}$ on top of the d.c. bias. In general the results were similar to those obtained by the previous work, showing three arcs with peak at high $(1 \mathrm{KHz})$, mid $(10 \mathrm{~Hz})$, and low $(0.3 \mathrm{~Hz})$ frequencies. However, the low frequency arc (previously assigned to the anode kinetics), appears to have grown by more than an order of magnitude.
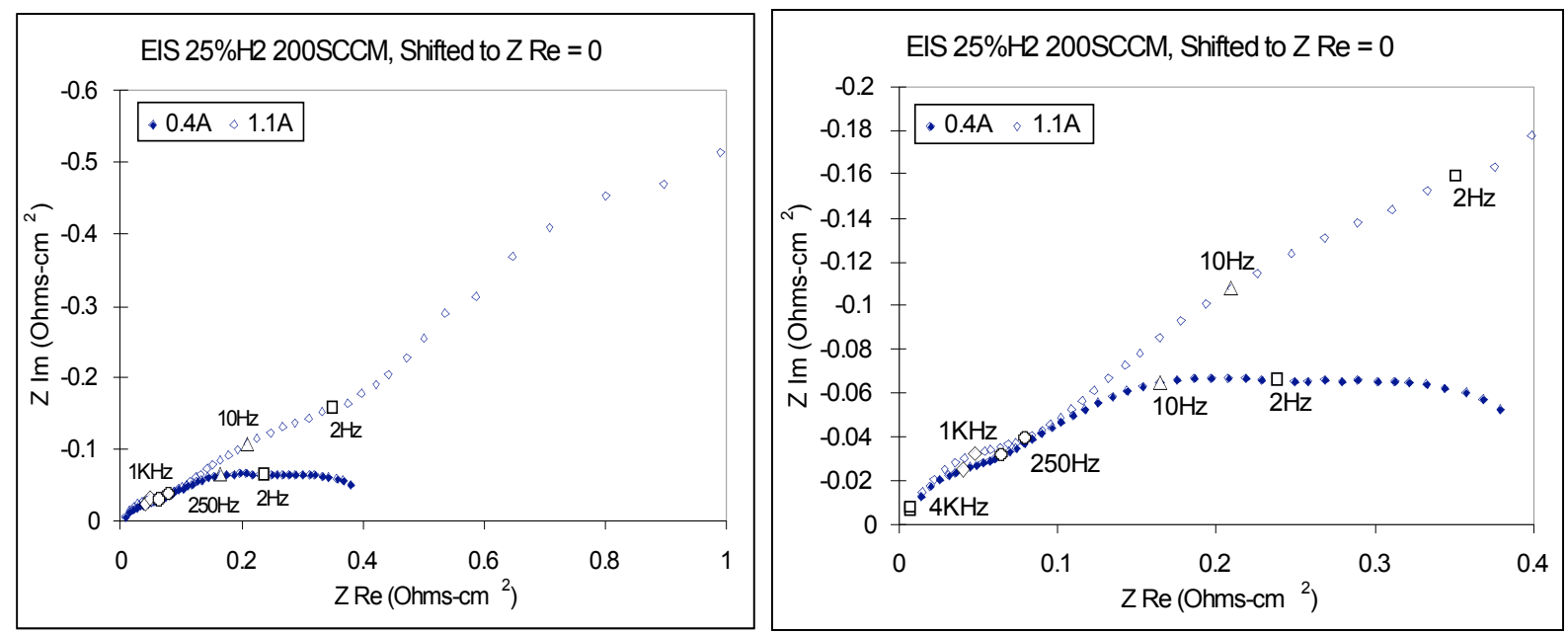

Figure B-11. EIS sweeps at low and high bias for the $25 \% \mathrm{H}_{2} 200 \mathrm{SCCM}$ gas condition. The figure at right is a magnification of the high frequency data.

In order to determine if a stagnant gas layer could explain the effects observed above, a FEMLAB model of fluid flow and Stefan-Maxwell diffusion above the cell was constructed based on the known geometry of the test stand (See Fig. B-12, right) ${ }^{[27]}$. As shown in Figure B13 , this model came close to predicting the observed limiting current, without any adjustable parameters.

In order to determine if NLEIS is sensitive to nonlinearities associated with gas-phase mass transfer (and capable of isolating those nonlinearities in the frequency domain), we conducted NLEIS measurements under both low polarization and high polarization conditions. Fig. B-13 (right) shows the results for the $2^{\text {nd }}$ harmonic (which was the only higher harmonic we could 
successfully isolate in this case). Note that on this scale the $2^{\text {nd }}$ harmonic at high frequency is very small, reflecting the relative linearity of the kinetic processes seen in the EIS spectrum. At low frequency, however, a much larger $2^{\text {nd }}$ harmonic develops that is tied in frequency to the low frequency arc in the EIS. The second harmonic switches sign from negative to strongly positive (off scale) as limiting current is approached.

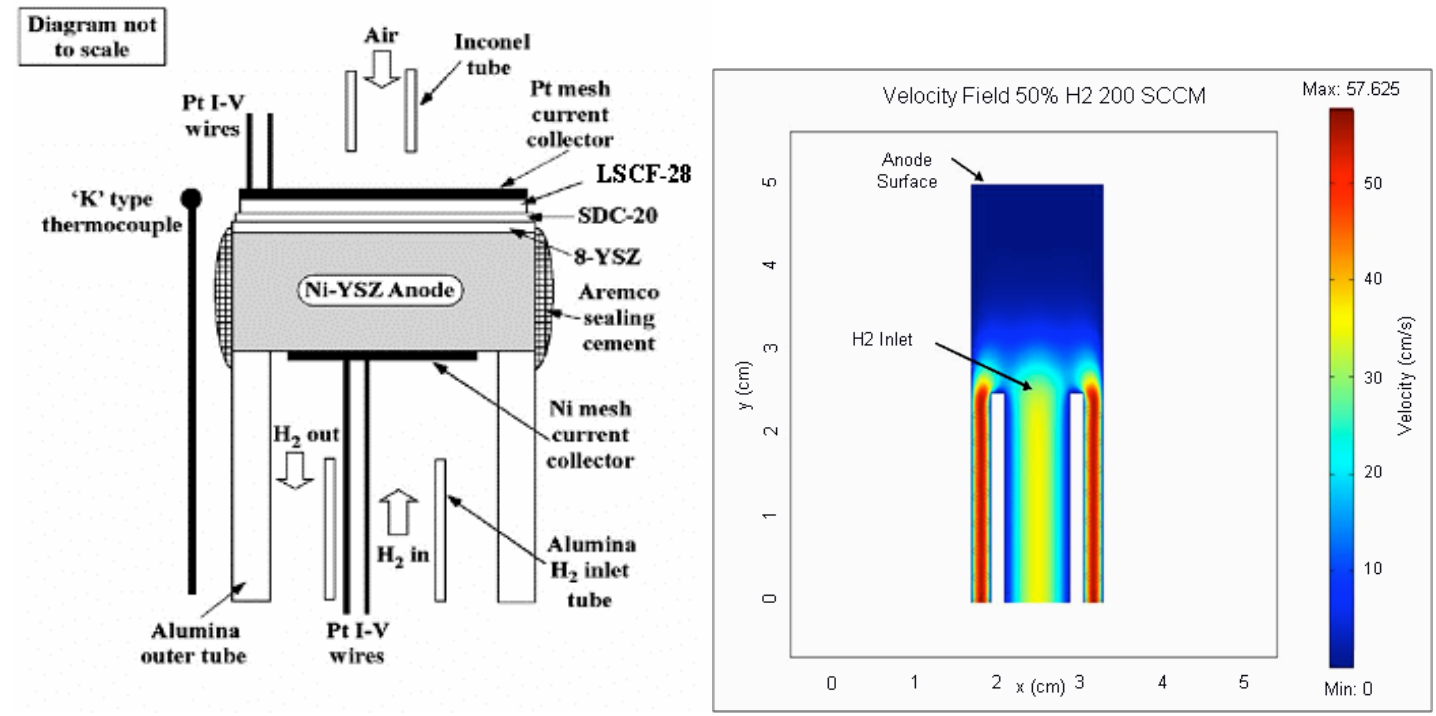

Fig. B-12. Finite-element calculation of fluid flow and mass transfer above a PNNL button cell under relevant conditions (see text).
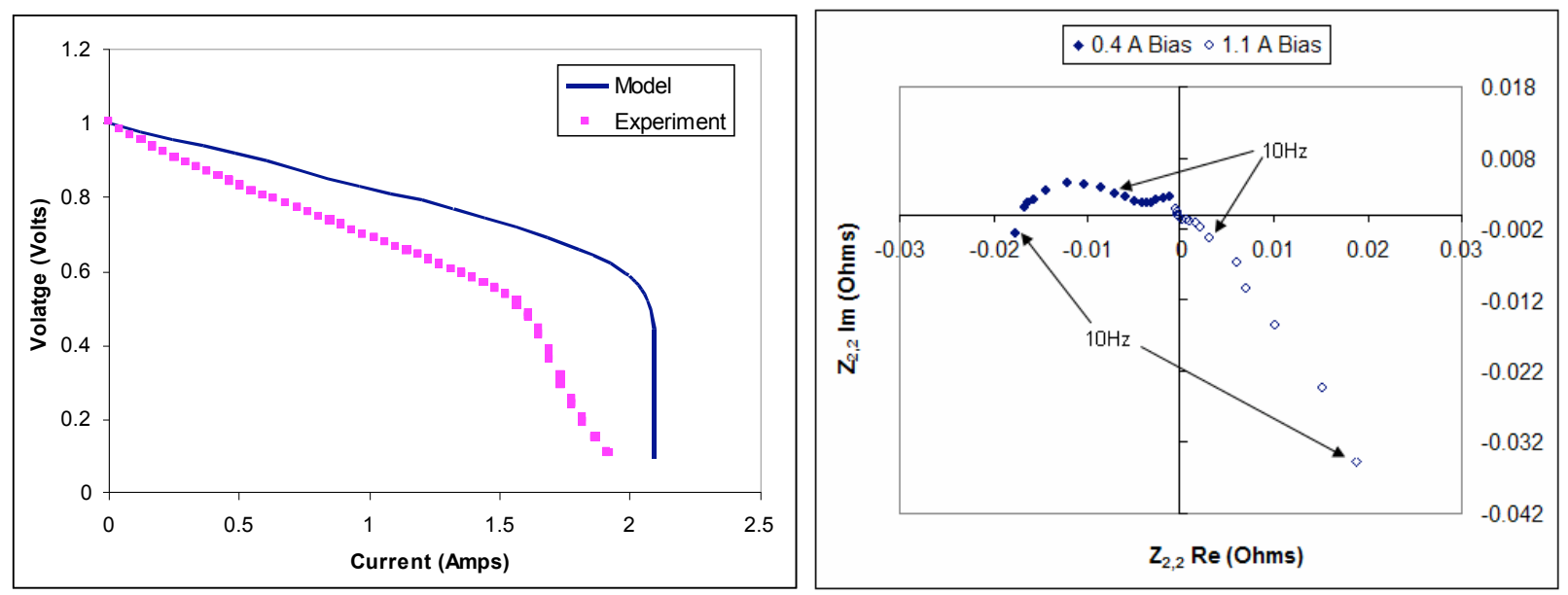

Fig. B-13. Left: Measured and calculated i-V curve for finite-element calculation of fluid flow and mass transfer above a PNNL button cell under relevant conditions (see text). Right: Second harmonic response under low and high polarizations, exhibiting a switch in sign at low frequency as predicted by the same model. 
This same model in Fig. B-12 can also be used to predict the harmonics associated with gasphase mass transfer in the limit of low frequency. The model predicts a sigmoidal gas-phase diffusion resistance, which results from the multicomponent diffusion of $\mathrm{H}_{2}$ and $\mathrm{H}_{2} \mathrm{O}$ in $\mathrm{N}_{2}{ }^{\text {[27] }}$. Unlike binary diffusion (which acts linear at small polarizations), the Stefan-Maxwell model predicts a small negative $2^{\text {nd }}$ order current dependence. At large polarizations the sign switches to positive, and becomes large near limiting current, reflecting the primary role of $\mathrm{H}_{2}$ starvation. Thus the NLEIS data further support the view that the low frequency arc is caused by a gaseousdiffusion effect. This example illustrates the potential power of NLEIS for isolating the nonlinearities associated with specific EIS features, and tying those nonlinearities to specific phenomena through modeling.

\section{B-6. Test-Bed Application \#3: Grain Boundary Effects in Oxide Electrolytes.}

One particular area of interest in studying solid oxide fuel cells (SOFC) is the interfacial transfer of oxygen ions between two adjacent solids. However, the nonlinearities of such processes have so-far been uncharacterizable, because interfacial charge-transfer typically occurs at relatively fast timescales, and thus is always obscured by low frequency processes.

As an example, Fig. B-14 shows a high frequency arc that sometimes appeared in our EIS studies of porous mixed-conducting cells. As explained in the figure caption, we believe this arc is associated with grain boundary conduction in ceria. Because grain-boundary conduction is usually considered ohmic, one might expect very little nonlinearity associated with it. However, because this arc was typically small compared to the electrode resistance under relevant conditions, it was not feasible to measure EIS as a function of polarization to ascertain any deviation this arc might have from linear response.
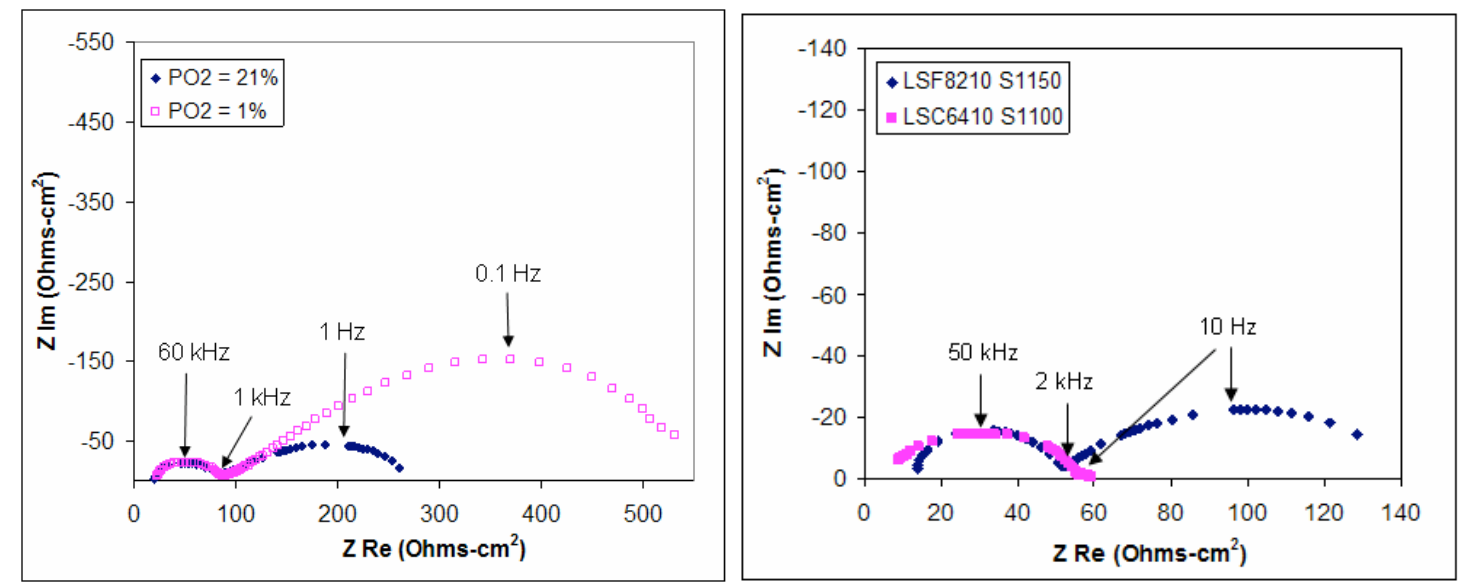

Fig. B-14. Examples of EIS spectra exhibiting a high frequency arc $(\sim 50 \mathrm{kHz})$ when the GDC electrolyte was fabricating using certain suppliers of nanocystalline GDC. Left: electrode is $\mathrm{La}_{0.8} \mathrm{Sr}_{0.2} \mathrm{FeO}_{3-\delta}$ (LSF-82) fired at $1100^{\circ} \mathrm{C}$, tested at $600{ }^{\circ} \mathrm{C}$ at two different $P_{O 2}$ 's. Right: cells with identical electrolyte and testing conditions $\left(610^{\circ} \mathrm{C}\right.$ in air), but different electrodes: LSF-82 sintered at $1150{ }^{\circ} \mathrm{C}$ and LSC-64 sintered at 1100 ${ }^{\circ} \mathrm{C}$. The very small capacitance of the high-frequency arc, as well as its insensitivity to $P_{O 2}$ and electrode type suggest it is related to grain boundary resistance in GDC ${ }^{[27]}$. 
In contrast, as shown in the previous test-bed application, NLEIS is uniquely capable of identifying nonlinearity associated with a particular timescale. In the present case the feature in question is well-separated in frequency, but is very small in magnitude, and thus serves as an ideal candidate for characterization using NLEIS.

High quality NLEIS data were obtained for a three-electrode cell consisting of nanocrsytalline GDC electrolyte sintered at $1600{ }^{\circ} \mathrm{C}$ with LSF-82 electrodes sintered at $1100^{\circ} \mathrm{C}$. Measurements were made in air $600{ }^{\circ} \mathrm{C}$. The cell produced a high-frequency arc with a peak frequency of $\sim 40 \mathrm{kHz}$, and a characteristic resistance of $\sim 120 \mathrm{Ohms}$. These values were used to nondimensionalize the higher harmonic data as described elsewhere ${ }^{[23][27]}$.

Fig. B-15 shows Nyquist Plots of the $2^{\text {nd }}$ and $3^{\text {rd }}$ harmonic spectra in the limit of high frequency. The data approach zero at high frequency (as expected for any model), and exhibit a distinct counterclockwise rotation toward the $2^{\text {nd }}$ quadrant. The magnitude of the harmonics is very small $(<0.1 \%)$, showing that measurements of EIS as a function of polarization would be unable to reveal such a small effects within the limits of noise. This result also shows that the grain boundary resistance is nearly linear (as expected), but not quite linear (which was not expected).
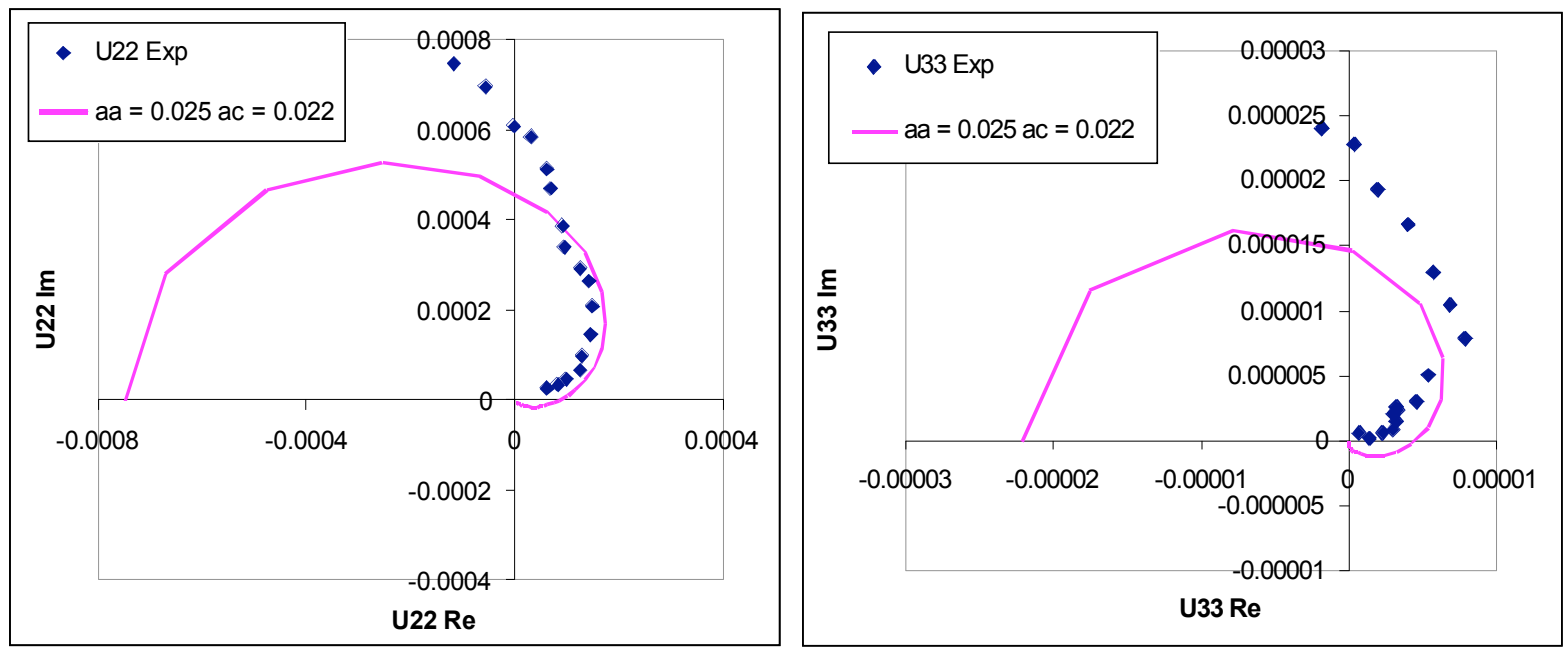

Fig. B-15. Nyquist plot of measured (symbols) and calculated (lines) $U_{2,2}$ and $U_{3.3}$ response for a $\mathrm{LSF} / \mathrm{GDC}$ cell at $600^{\circ} \mathrm{C}$ in air. Model calculations are for $\alpha_{\mathrm{a}}$ and $\alpha_{c}$ values of 0.025 and 0.022 , respectively, as explained in the text. Such small and symmetrical transfer coefficients is consistent with ion hopping over a distance of $\sim 50$ lattice plains. 


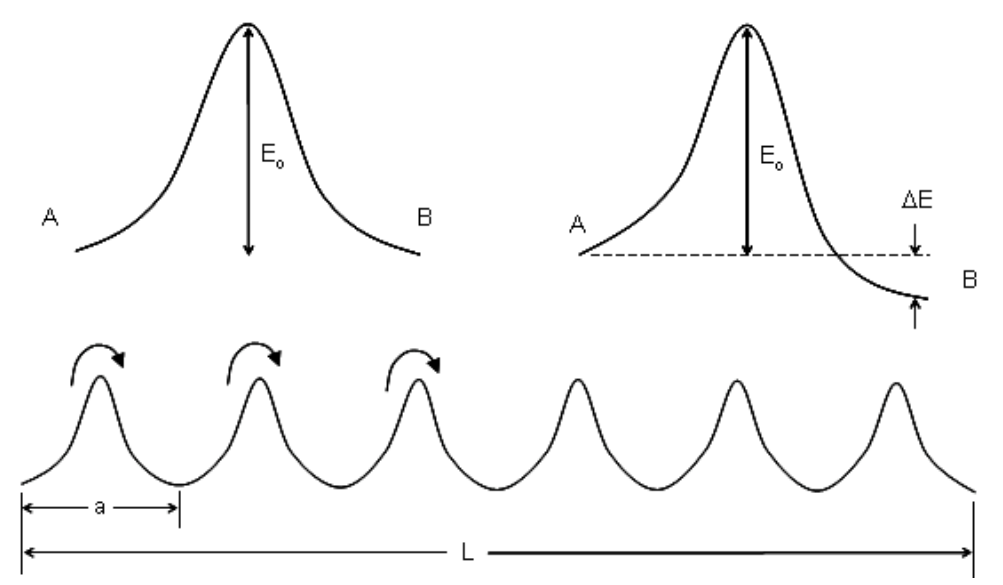

Fig. B-16. Model for ion hopping used to explain the data in Fig. B-15, above. Each ion hopping event involves an activation energy, which is perturbed by application of an electric field. The resulting net hopping rate follows Butler-Volmer kinetics, where anodic and cathodic transfer coefficients are inversely proportional to the number of lattice plains crossed by an ion along the conduction path ${ }^{[27]}$.

In order to explain the existence of small nonlinearities in the grain-boundary resistance, we developed the model shown in Fig. B-16 for the ion hopping process in a regular 1-D lattice where a voltage driving force is applied across nanoscale distances $(<100$ lattice planes). Under these conditions, a significant fraction of applied voltage can act on a single hop, which if modeled in terms of transition state theory predict a rate given by ${ }^{[27]}$ :

$$
i=z_{i} k c_{i} e^{\frac{-E_{o}}{R T}}\left(e^{z_{i} \frac{V F a}{L 2 R T}}-e^{-z_{i} \frac{V F a}{L 2 R T}}\right)+C \frac{\partial V}{\partial t}
$$

where $z_{i}$ is the ion charge, $c_{i}$ is the ion concentration, $k$ is a pre-exponential rate factor, $E_{a}$ is hopping activation energy, $V$ is applied voltage, $L$ is the total thickness of grain boundary material that current must travel through, a is the hop distance, and $C$ is the total grain boundary capacitance. Note that this equation has the same form as the Butler-Volmer equation, where we replace the anodic and cathodic transfer coefficients by $z_{i} a / 2 L$ :

$$
i=i_{o}\left(e^{\alpha_{a} \frac{V F}{R T}}-e^{-\alpha_{c} \frac{V F}{R T}}\right)+C \frac{\partial V}{\partial t}
$$

Calculation of the nondimensional higher harmonics predicted by eqn. B-5 yields:

$$
\begin{aligned}
& U_{1,1}(\omega)=\frac{j}{2 j-2 \omega} \\
& U_{2,2}(\omega)=-\frac{j\left(\alpha_{a}-\alpha_{c}\right)}{(2 j-2 \omega)^{2}(-2 j+4 \omega)} \\
& U_{3,3}(\omega)=\frac{-2 \alpha_{a}^{2}(j+\omega)-2 \alpha_{c}^{2}(j+\omega)+\alpha_{a} \alpha_{c}(5 j+2 \omega)}{48(-j+\omega)^{3}\left(-1-5 j \omega+6 \omega^{2}\right)}
\end{aligned}
$$


These equations show that the first harmonic is insensitive to the transfer coefficients $\alpha_{a}$ and $\alpha_{c}$. However, the second and third harmonics allow us to triangulate their values. Specifically, the weak $2^{\text {nd }}$ harmonic in this case suggests the kinetics are nearly symmetric, and thus $\alpha_{a}$ and $\alpha_{c}$ have similar values. A best fit of eqns. B-6(a c) to the data in Fig. B-15 yield values for $\alpha_{a}$ and $\alpha_{c}$ of 0.022 and 0.025 respectively. Agreement between model and data breaks down at lower frequencies where the much larger nonlinearities in this case of the electrode take over.

The small value of $\alpha_{a}=\alpha_{c}$ revealed above would correspond to $L / a \sim 50$ lattice planes assuming $\mathrm{O}^{2-}$ ions as the transporting species. SEM examination of the sample shows that significant grain growth occurs at $1600^{\circ} \mathrm{C}$ firing conditions. Based on the position of the reference electrode, we estimate the current must travel through approximately 100 grains. Given the possibility that some grains may be better connected than others, this seems like reasonable agreement. In other words, it appears that NLEIS is sufficiently sensitive to detect the very small nonlinearity of symmetrical hoping of oxygen ions among grains in an electrolyte sample.

\section{B-7. Summary.}

This section described out development of nonlinear electrochemical impedance spectroscopy (NLEIS), a variant of traditional impedance that allows workers to probe rate nonlinearity as a function of frequency. The three test-bed applications described above demonstrate the ability of NLEIS to isolate nonlinearities specific to particular timescales, and thus provide more information than is available from linear response alone. It was demonstrated that NLEIS signals could be obtained for porous SOFC cathodes, and that the higher harmonics could be used to distinguish proposed empirical rate expression for the kinetics. The use of NLEIS to probe mass-transfer effects in a two-compartment fuel cell at PNNL was also shown. Finally, we demonstrated that the very small nonlinearities associated with grain-boundary conduction in ceria could be isolated, and explained quantitatively in terms of a charge-transfer model involving a quantized series of ionic interfaces.

The following section brings together many of the ideas demonstrated above, applying them to a well-defined and characterized model electrode geometry. It is shown that the small nonlinearities associated with oxygen exchange on a mixed conductor can be used to eliminate candidate mechanisms, and identify the rate-limiting atomic steps governing kinetics. 


\section{Part II: Studies of Reaction and Degradation Mechanisms Governing $\mathrm{La}_{1-\mathrm{x}} \mathrm{Sr}_{\mathrm{x}} \mathrm{CoO}_{3-\delta}$ (LSC) on Gd-doped Ceria.}

\section{Studies of Thin Film LSC Electrodes}

\section{C-1. Introduction}

Little is currently known about the mechanisms or rate laws governing $\mathrm{O}_{2}$ exchange at the gas-exposed surface of a mixed-conductor. In the limit of equilibrium, the rate of $\mathrm{O}_{2}$ exchange with the bulk has been studied using a variety of methods, including step-relaxation measurements ${ }^{[28-37]}$ and isotope tracer techniques ${ }^{[38-41]}$. By measuring the exchange rate over an array of conditions (temperature, oxygen partial pressure, dopant concentration), workers have tried to infer the mechanism of the reaction. However, this class of measurements suffers from two fundamental limitations. First, it is often difficult to separate surface exchange rates from bulk transport rates over a sufficiently wide range of conditions. Secondly, and more significantly, these measurements are only able to isolate the linearized rate law near equilibrium. Linearized rates are an inherently weak indicator of mechanism, since numerous mechanisms can often explain the same linearized rate coefficient with the appropriate choice unknown adjustable parameters ${ }^{[42]}$.

In the case of the perovskite mixed conductors $\mathrm{La}_{1-\mathrm{x}} \mathrm{Sr}_{\mathrm{x}} \mathrm{Co}_{\mathrm{y}} \mathrm{Fe}_{1-\mathrm{y}} \mathrm{O}_{3-\delta}$, workers have successfully addressed the first of these problems by making dense thin film electrodes ${ }^{[13,43-47]}$. These films are often sufficiently thin $(300 \sim 1000 \mathrm{~nm})$ that bulk transport remains equilibrated, and thus interfacial rates can be isolated. By employing electrochemical impedance spectroscopy (EIS), workers have been able to further isolate the $\mathrm{O}_{2}$ exchange rate from other interfacial processes. In addition to rate, EIS also provides information about driving force, which must also be known in order to describe overall kinetics in terms of specific elementary steps and species ${ }^{[42]}$. For example, Kawada and coworkers ${ }^{[45]}$ analyzed the capacitive properties of dense thin film $\mathrm{La}_{0.6} \mathrm{Sr}_{0.4} \mathrm{CoO}_{3-\delta}$ (LSC-64) in terms of the non-equilibrium displacement of oxygen stoichiometry from equilibrium. Taken together, the resistive and capacitive properties of the film reveal the linear relationship between $\mathrm{O}_{2}$ reduction/oxidation and displacement of vacancy concentration from equilibrium under small driving forces.

However, as mentioned above, the linearized kinetics provided by EIS are insufficient information to fully assess mechanism, even when performed as a function of steady-state polarization. To address this second problem, we introduce in this paper the use of nonlinear impedance spectroscopy (NLEIS) ${ }^{[17,48]}$ on thin-film electrodes to more fully probe $\mathrm{O}_{2}$ exchange kinetics at moderate displacements from equilibrium. As with traditional impedance, NLEIS separates processes via timescale; but unlike impedance, NLEIS selectively detects nonlinearities associated with individual features in the EIS spectrum. When modeled using nonlinear rate expressions based on irreversible thermodynamics ${ }^{[42]}$, these harmonics can be used to distinguish mechanisms that would otherwise predict degenerate linear response. Although other electrochemical techniques probe nonlinear rates (e.g. $i$ - $V$ characteristics, cyclic voltammetry, EIS as a function of polarization), NLEIS has a unique ability to isolate nonlinearities specific to phenomena occurring at a particular timescale. NLEIS also has an advantage in detecting very 
small nonlinearities ( $<1 \%$ of linear response) through Fourier analysis, which would otherwise be indistinguishable from performance drift or noise uncorrelated to the perturbing signal. This approach is applied here to study a thin film of $\mathrm{La}_{0.6} \mathrm{Sr}_{0.4} \mathrm{CoO}_{3-\delta}$ electrodes on polycrystalline Gddoped ceria, a system with well-defined thermodynamics that has been shown previously to be limited primarily by the $\mathrm{O}_{2}$ exchange reaction ${ }^{[45]}$.

\section{C-2. Experimental}

$\mathrm{Ce}_{0.9} \mathrm{Gd}_{0.1} \mathrm{O}_{1.95}$ powder was made by coprecipitation of $\mathrm{Ce}$ and $\mathrm{Ge}$ oxalates from nitrate salts, and calcinated at $800^{\circ} \mathrm{C}$. The powder was pressed into a pellet and sintered at $1550^{\circ} \mathrm{C}$ for 6 hours, then polished with diamond paste, resintered at $1550 \mathrm{C}$ for 2 hours, and finally polished a second time. The resulting pellet was approximately $0.2 \mathrm{~cm}$ thick, and $1 \mathrm{~cm}$ in diameter.

The thin film $\mathrm{La}_{0.6} \mathrm{Sr}_{0.4} \mathrm{CoO}_{3-\delta}$ electrode was deposited onto the $\mathrm{Ce}_{0.9} \mathrm{Gd}_{0.1} \mathrm{O}_{1.95}$ pellet surface by Pulse Laser Deposition (PLD), with a $308 \mathrm{~nm} \mathrm{XeCl} \mathrm{eximer} \mathrm{laser,} \mathrm{operating} \mathrm{at} 10 \mathrm{~Hz}$. The $\mathrm{La}_{0.6} \mathrm{Sr}_{0.4} \mathrm{CoO}_{3-\delta}$ target was fabricated from $\mathrm{La}_{0.6} \mathrm{Sr}_{0.4} \mathrm{CoO}_{3-\delta}$ powder, from Praxair Specialty Ceramics, calcined at $1100^{\circ} \mathrm{C}$ for 4 hours and then sintered at $1200^{\circ} \mathrm{C}$ for 5 hours. The cell was held at $700^{\circ} \mathrm{C}$, and $10^{-5}$ atm $\mathrm{O}_{2}$ during deposition. After deposition, the cell was annealed at $700^{\circ} \mathrm{C}$ and 1 atm for 3 hours. The film was examined with scanning electron microscopy (SEM), and some cracks of widths between 50 and $100 \mathrm{~nm}$ were observed, but not covering a significant fraction of the surface.

The cell was configured with a porous Pt counterelectrode, and a Pt reference electrode (wire), wrapped around the circumference of the pellet. Gold mesh current collectors were applied to the working and counter electrodes to ensure contact, and proper current distribution. The cell was placed in a NorECs Probostat for EIS and NLEIS measurements, where a springloaded alumina assembly held the cell and gold current collectors in place. EIS and NLEIS measurements were performed at $725^{\circ} \mathrm{C}$ in blended gases of $\mathrm{O}_{2}$ in $\mathrm{N}_{2}$ spanning $P_{O 2}=10^{-3} \sim 0.21$ atm. NLEIS measurements were performed at 15 perturbation amplitudes per perturbation frequency, with 10 frequency points per decade between 0.01 and 10,000 Hz. Excessive noise levels made calculation of the higher harmonics impossible below $P_{O 2} \sim 10^{-2} \mathrm{~atm}$. Significant drift in the first harmonic (decreasing impedance) was observed over the course of the experiments, particularly at the lowest frequencies $(0.01 \mathrm{~Hz})$ where signal averaging required several hours to complete.

\section{C-3. Film Characterization}

Figure C-1 shows scanning electron microscopy (SEM; FEI Sirion) images of the $\mathrm{La}_{0.6} \mathrm{Sr}_{0.4} \mathrm{O}_{3-\delta}$ (LSC-64) film on $\mathrm{Ce}_{0.9} \mathrm{Gd}_{0.1} \mathrm{O}_{1.95}$ (GDC) following impedance measurements. LSC-64 film thickness was examined by optical profilometry and scanning electron microscopy (SEM), and found to have a uniform thickness of $\sim 900 \mathrm{~nm}$, except for what appear to be precipitates of LSC on the surface. These precipitates form during electrochemical measurement, and may be a partial explanation for a decreasing drift in impedance over the course of the experiments (to be published). X-ray diffraction measurements on similar films made at Tohoku University suggests that the perovskite lattice is has no preferred orientation in 
the plane of the polycrystalline substrate, but is preferentially oriented in the 001 direction (of the pseudo-cubic lattice) perpendicular to the substrate (to be published).
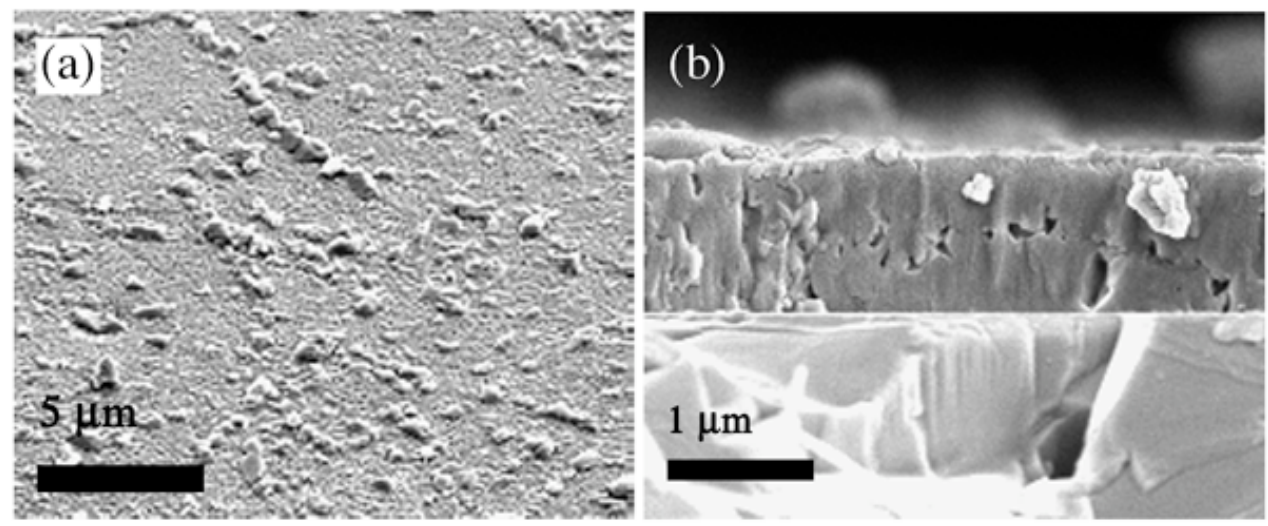

Fig. C-1: SEM micrographs of thin film $\mathrm{La}_{0.6} \mathrm{Sr}_{0.4} \mathrm{CoO}_{3-\delta}$ on Gadolinia doped Ceria. (a) Top surface of $\mathrm{La}_{0.6} \mathrm{Sr}_{0.4} \mathrm{CoO}_{3-\delta}$ following EIS and NLEIS experiments. (b) Fracture cross-section showing electrode/electrolyte interface and nominal electrode thickness ( $900 \mathrm{~nm})$.

\section{C-4. Linear Response}

Fig. C-2a shows a Nyquist plot of the linear impedance (EIS) response. As found previously ${ }^{[45]}$, the electrode impedance consists primarily of a low frequency $P_{O 2}$-dependent semicircle attributable to the electrochemical reaction $\frac{1}{2} \mathrm{O}_{2}+2 e^{-} \rightleftharpoons \mathrm{O}^{2-}$. (A much smaller high frequency arc is also observed, but not examined in this paper). This impedance could be fit with excellent precision to a RC circuit model:

$$
Z(\omega)=\frac{R_{\text {chem }}}{1+j \omega R_{\text {chem }} C_{\text {chem }}},
$$

with the $P_{O 2}$ dependence of $R_{\text {chem }}$ and $C_{\text {chem }}$ summarized in Fig. C-2b.

As shown elsewhere (Reference ${ }^{[42]}$, eqn. 22), the equilibrium oxygen exchange rate is inversely proportional to $R_{\text {chem. }}$. A fit of $\log \left(R_{\text {chem }}\right)$ vs. $\log \left(P_{O 2}\right)$ could be fit approximately to a straight line with slope $n=-\partial \ln R_{\text {chem }} / \partial \ln P_{O_{2}}=0.41$, as shown in Fig. C-2b. Although this value falls in a reasonable range for oxygen exchange on LSC, we have shown previously that $n$ is a very poor indicator of mechanism when only considered over the narrow range of $P_{O 2}$ examined here ${ }^{[42]}$.

Using procedures outlined previously ${ }^{[45]}$, the faradaic (chemical) capacitance of the film, $C_{c h e m}$, was also examined to determine the vacancy concentration within the film. Based on Faraday's law, this procedure involves fitting $C_{c h e m}$ vs. $P_{O 2}$ to a nonstoichiometry model (proposed originally by Mizusaki ${ }^{[49]}$, and later refined by Lankhorst ${ }^{[50]}$ ) that assumes electrons are ideally delocalized. This itinerant electron model has been found to explain the nonstoichiometry properties of LSC over a wide range of $T, P_{O 2}$, and $\mathrm{Sr}$ content ${ }^{[49,51]}$. The best 
fit of $C_{\text {chem }}$ extracted from Fig. C-2a to the itinerant electron model is shown with the data in Fig. $\mathrm{C}-2 \mathrm{~b}$. (The values of the parameter, defined in reference ${ }^{[45]}$, are: $\Delta s_{O}(x)=-69.5 \mathrm{~J} / \mathrm{mol}-\mathrm{K}$, $\Delta h_{O}(x)=-116 \mathrm{~kJ} / \mathrm{mol}$, and $\left.a(x)=338 \mathrm{~kJ} / \mathrm{mol}\right)$. This same fit can be re-expressed as the nonstoichiometry $\delta$ (in $\mathrm{La}_{0.6} \mathrm{Sr}_{0.4} \mathrm{CoO}_{3-\delta}$ ) vs. $P_{O 2}$, as re-plotted in Fig. C-2c (solid black line). As found previously, the nonstoichiometry determined in this manner was found to be lower than for bulk LSC-64 - a possible consequence of film strain due to lattice mismatch with the underlying substrate ${ }^{[45]}$.
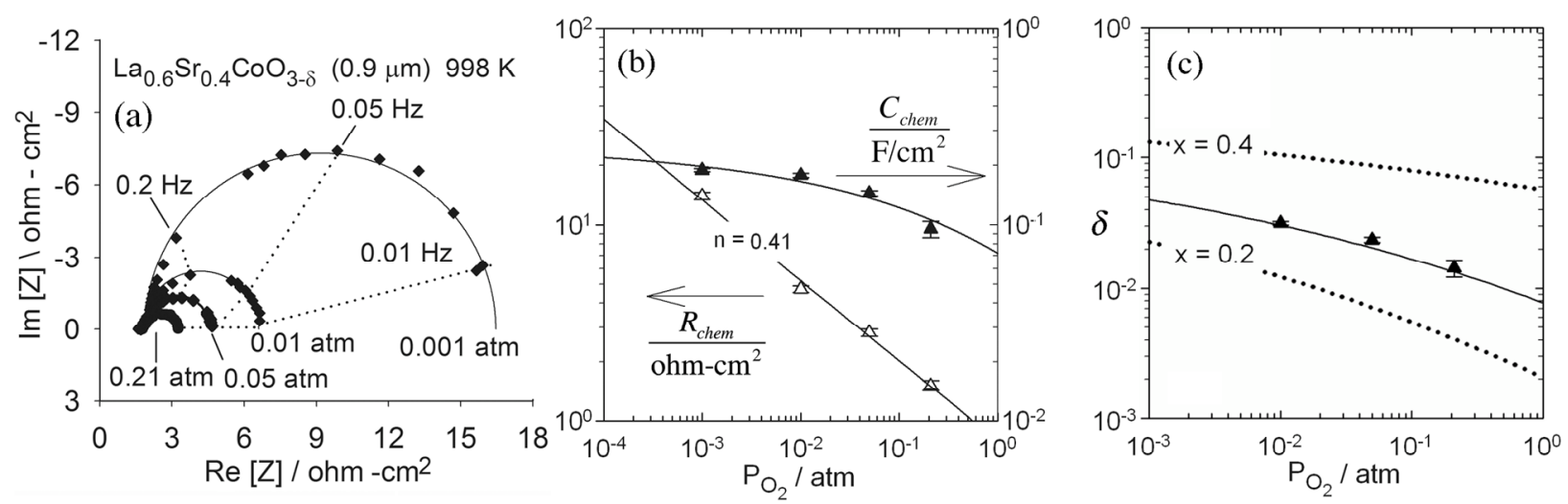

Fig. C-2: (a) Nyquist plots of impedance of thin film $\mathrm{La}_{0.6} \mathrm{Sr}_{0.4} \mathrm{CoO}_{3-\delta}$, measured at zero current bias and $725{ }^{\circ} \mathrm{C}$ as a function of oxygen partial pressure. Solid lines are fits to eqn. C-1. (b) Values of $R_{\text {chem }}$ (open symbols) and $C_{\text {chem }}$ (closed symbols) extracted from the data in (a). The solid line through $R_{\text {chem }}\left(P_{O_{2}}\right)$ is an empirical fit to a power law. The solid line through $C_{c h e m}\left(P_{O 2}\right)$ is a best fit to an oxygen nonstoichiometry model, as explained in the text. (c) Calculated nonstoichiometry $\delta$ vs. $P_{O 2}$ based on the fit to $C_{\text {chem }}$ in (b) (solid line). The dashed lines are best fits of the same model to measured nonstoichiometry of bulk $\mathrm{La}_{0.6} \mathrm{Sr}_{0.4} \mathrm{CoO}_{3-\delta}$ and $\mathrm{La}_{0.8} \mathrm{Sr}_{0.2} \mathrm{CoO}_{3-\delta}{ }^{[45]}$. The data points are a separate calculation of $\delta$ vs. $P_{O 2}$ in the thin film based on the higher harmonics, as explained in the text.

\section{C-5. Nonlinear Response}

During the same experimental session as the EIS measurements, NLEIS measurements were performed using procedures outlined in detail elsewhere ${ }^{[48]}$. Part of this procedure requires scaling the voltage $(V)$, current density perturbation amplitude $(\tilde{i})$, and perturbation frequency ( $\tilde{\omega})$ to characteristic values: $U=V / V^{*}, \alpha=\tilde{i} / i^{*}$, and $\sigma=\tilde{\omega} / \omega^{*}$, respectively, based on the first harmonic (impedance). In this case we defined:

$$
V^{*}=\frac{R T}{2 F} ; \quad i^{*}=\frac{V^{*}}{R_{\text {chem }}} ; \quad \omega^{*}=\frac{1}{R_{\text {chem }} C_{\text {chem }}} .
$$

Amplitude-independent and dimensionless harmonic spectra $\hat{U}_{1,1}(\sigma), \hat{U}_{2,2}(\sigma)$ and $\hat{U}_{3,3}(\sigma)$ were then extracted from the $1^{\text {st }}, 2^{\text {nd }}$ and $3^{\text {rd }}$ harmonic voltages as described previously ${ }^{[48]}$, 
applying the scaling in eqn. C-2. The benefit of scaling is that the magnitude of $\hat{U}_{m, m}(\sigma)$ gives an idea of the size of the harmonic nonlinearity relative to the impedance, while the dimensionless frequency $\sigma=\omega / \omega^{*}$ allows the timescale of the harmonic response to be compared to the characteristic time constant of the electrode $\left(R_{\text {chem }} C_{\text {chem }}\right)$.
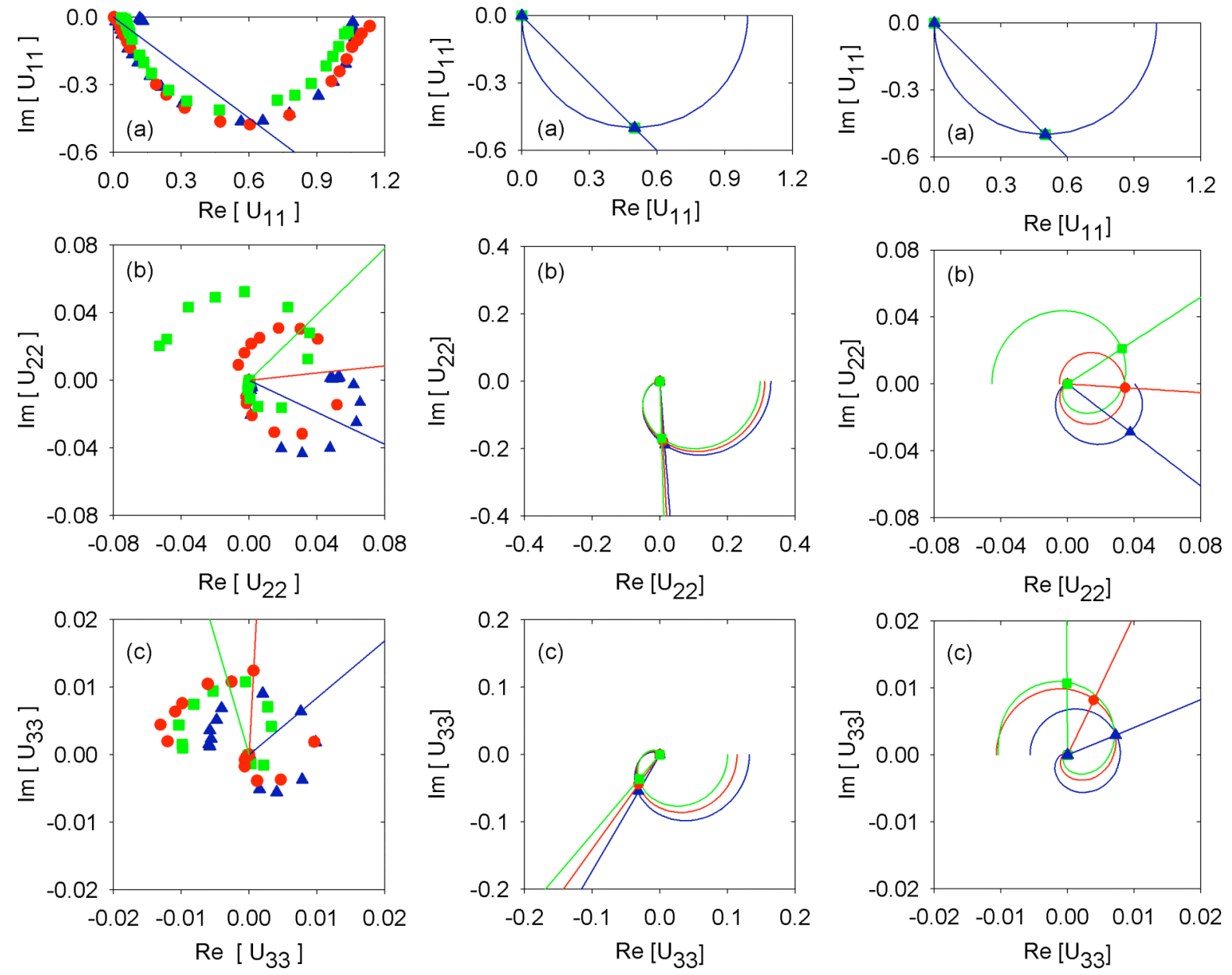

Fig. C-3: Left: Nyquist plots of the experimental harmonic spectra $\hat{U}_{1,1}$ (a), $\hat{U}_{2,2}$ (b) and $\hat{U}_{3,3}(\mathrm{c})$ (symbols) as a function of frequency under different oxygen gas environments: $1 \% \mathrm{O}_{2}$ (blue/dark triangles), $5 \% \mathrm{O}_{2}$ (red/medium gray circles) and 21 $\% \mathrm{O}_{2}$ (green/light gray squares) in diluent $\mathrm{N}_{2}$ at $725{ }^{\circ} \mathrm{C}$. The phasors extending from the origin cross the data where the dimensionless frequency $(\sigma)$ has a value of $1,1 / 2$ and $1 / 3$, respectively, for $\hat{U}_{1,1}, \hat{U}_{2,2}$ and $\hat{U}_{3,3}$. Middle: Calculated harmonic spectra (lines) as a function of thermodynamic factor $\gamma$ for the mechanistic scenario in Fig. C4a. Blue/dark: $\gamma=-0.27$; red/medium gray: $\gamma=-0.25$; Green/light gray: $\gamma=-0.23$. Right: Calculated harmonic spectra for the mechanistic scenario in Fig. C-4b. 
Figure C-3a (left column) shows a Nyquist plot of the dimensionless first harmonic spectrum $\hat{U}_{1,1}(\sigma)$ as a function of $P_{O 2}$. The data reveal a $P_{O 2}$-independent semicircle of width 1 and negative imaginary maximum at $\sigma=1$, as achieved by the scaling in eqn. C-2. The scaling parameters $R_{\text {chem }}$ and $C_{\text {chem }}$ were found to be in good agreement with the impedance measured at low amplitude (Fig 2a), although overall electrode resistance drifted approximately $10 \%$ over the course of the NLEIS experiments.

Figures C-3b and C-3c (left column) show Nyquist plots of $\hat{U}_{2,2}(\sigma)$ and $\hat{U}_{3,3}(\sigma)$ vs. $P_{O 2}$. For each $P_{O 2}$, a radial reference line (phasor) crosses the data where the dimensionless frequency $\sigma$ is equal to $1 / m$, where $m$ is the order of the harmonic $\hat{U}_{m, m}$. Two things are notable about this data. First, the shape, magnitude, and phase of the $2^{\text {nd }}$ and $3^{\text {rd }}$ harmonic spectra are all highly sensitive to $P_{O 2}$ over a small range ( $1 \%$ to $21 \%$ oxygen). This is quite different than the impedance (Fig 3a), which is entirely $P_{O 2}$-independent after scaling. The data exhibit a distinct counterclockwise rotation of the $2^{\text {nd }}$ and $3^{\text {rd }}$ harmonic phasors with decreasing $P_{O 2}$, including a nullification (sign change) in the $2^{\text {nd }}$ harmonic with $P_{O 2}$ at low frequency near $P_{O 2}=0.05$ atm. As shown below, these very distinct dependencies can be tied to nonlinear terms governing the kinetics.

The second notable observation is that the measured magnitudes of the harmonics shown in Fig. C-3 are quite small compared to the linear response: on the order of $5 \%$ for the $2^{\text {nd }}$ harmonic and $1 \%$ for the third. By comparison, the noise level seen in the impedance data (Fig. C-2a) is on the order of $\pm 3 \%$, while the signal drift observed over the entire course of these experiments was found to be on the order of $10 \%$. Thus the nonlinearities represented in Fig. C-3 would have been difficult to resolve with any accuracy from $i-V$ characteristics or EIS as a function of polarization. Indeed, ongoing work at Tohoku University (to be published) suggests that the film undergoes irreversible (or hysteretic) changes in morphology, surface structure, and/or surface composition as a function of time and/or polarization history. Even if one assumes these changes do not alter the underlying reaction mechanism, they nonetheless alter the response magnitude and timescale of the impedance, making it difficult to resolve the very small nonlinearities associated with the kinetic rate law itself. In contrast, NLEIS naturally filters out noise and performance drift because it only detects nonlinear signals directly correlated to the input signal. In this way NLEIS is rather like nuclear magnetic resonance spectroscopy (NMR), which employs signal averaging and Fourier analysis to detect signal correlations many orders of magnitude smaller than ambient noise or non-resonant signals.

\section{C-6. Modeling}

In attempting to explain the nonlinearities shown in Figure C-3, we examined a variety of nonlinear rate expressions for $\mathrm{O}_{2}$ exchange, corresponding to specific mechanisms and rate limiting steps ${ }^{[42]}$. Based on non-equilibrium thermodynamics and transition state theory, these rate expressions are rigorously consistent with thermodynamics, yet also treat large displacements from equilibrium. Assuming the existence of a rate-limiting elementary step, the rate of $\mathrm{O}_{2}$ reduction/oxidation can be expressed as: 


$$
r=\Re_{0}\left[1-e^{\frac{-\Lambda}{\lambda R T}}\right],
$$

where $r$ is the net $\mathrm{O}_{2}$ reduction rate at the surface, $\Lambda=R T \ln \left(P_{O_{2}} / f_{O_{2}}\right)$ is total free energy driving force for the reaction, $P_{\mathrm{O}_{2}}$ and $f_{\mathrm{O}_{2}}$ are the oxygen fugacities in the gas and solid phases, respectively, $\lambda$ is an integer parameter related to the rate-limiting step, and $\mathfrak{R}_{0}\left(P_{\mathrm{O}_{2}}, f_{\mathrm{O}_{2}}\right)$ is a kinetic function that depends on the thermodynamic conditions of both the gas and solid ${ }^{[42]}$. In the limit of equilibrium, $\mathfrak{R}_{0}$ becomes the exchange rate. The functional form of $\mathfrak{R}_{0}$ and values of $\lambda$ for various rate-limiting steps are summarized in Table 2 of reference ${ }^{[42]}$.
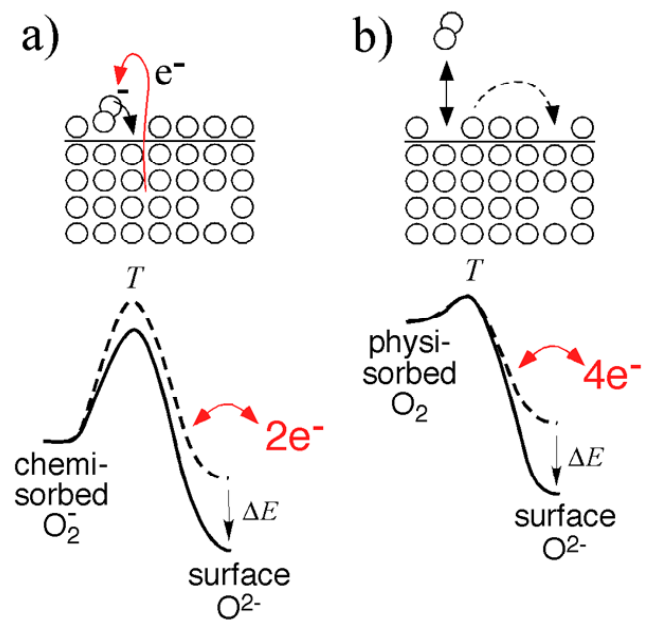

Fig. C-4: Two commonly-held theories for the rate-limiting step for $\mathrm{O}_{2}$ reduction on mixed conductors. In (a) the reaction is assumed limited by an energy barrier to dissociation of chemisorbed $\mathrm{O}_{2}$. In (b), the reaction is assumed limited by dissociative adsorption on limited surface sites. Although there is no energy barrier to the reaction, adjacency of a second surface vacancy within the physisorption lifetime of $\mathrm{O}_{2}$ is required to achieve dissociation (entropy barrier). See reference ${ }^{[42]}$ for further details.

Two specific mechanisms considered in Reference ${ }^{[42]}$ are illustrated in Fig. C-4. In the first (Fig. C-4a), it is assumed that $\mathrm{O}_{2}$ first forms a chemisorbed intermediate (superoxide) on the surface. The rate-limiting step is assumed to involve an energy barrier, which limits the dissociation of this intermediate. In the second (Fig. C-4b), no energy barrier is considered. The rate-limiting step is assumed to involve dissociative adsorption of $\mathrm{O}_{2}$ (as $\mathrm{O}^{2-}$ ) on a limited number of vacant surface oxygen sites. A full discussion of these mechanisms, the assumptions and parameters involved, and the derivation of $\mathfrak{R}_{0}\left(P_{\mathrm{O}_{2}}, f_{\mathrm{O}_{2}}\right)$ can be found in reference ${ }^{[42]}$.

For each candidate mechanism (and corresponding rate law in eqn. C-3), the harmonics were calculated by considering a simple stirred-tank model for the thin film (which was assumed to be diffusionally equilibrated). Conservation of oxygen in the film implies: 


$$
L c_{0} \frac{d x_{v}}{d t}=\left.N_{v}\right|_{y=0}-\left.N_{v}\right|_{y=L}
$$

where $x_{V}=\delta / 3$ is the oxygen vacancy concentration (nonstoichiometry) expressed as a mole fraction, $L$ is the thickness of the film, $c_{0}$ is the concentration of oxygen lattice sites, $t$ is time, $y$ is position within the electrode (as measured from the electrode/electrolyte interface), and $N_{v}$ is the molar flux of oxygen vacancies in the $y$-direction. The vacancy flux at the electrolyte and gas boundaries are determined by Faraday's law and eqn. C-3, respectively:

$$
\left.N_{V}\right|_{y=0}=-\frac{\tilde{i} \cos (\tilde{\omega} t)}{2 F} ;\left.\quad N_{V}\right|_{y=L}=2 r
$$

where $\tilde{i} \cos (\tilde{\omega} t)$ is the sinusoidal current density perturbation, and $r$ is defined by eqn. C-3. Finally, in the absence of interfacial resistance or gradients within the film, the voltage relative to a reference electrode of the same type is related to the film nonstoichiometry by the Nernst equation $^{[48]}$ :

$$
V=\frac{R T}{4 F} \ln \frac{f_{O_{2}}\left(x_{v}\right)}{P_{O_{2}}},
$$

where the solid oxygen fugacity $f_{O_{2}}\left(x_{v}\right)$ is related to $x_{v}$ using the itinerant electron model discussed previously ${ }^{[42]}$. Solution of eqns. C-2 $\sim$ C- 6 using the procedures outlined in reference ${ }^{[48]}$ yields calculated spectra for the first and higher harmonics $\hat{U}_{m, m}$.

After assuming a specific mechanism (as in Figs. C-4a or C-4b), the scaled higher harmonics only depend on one physical parameter that varies with $P_{\mathrm{O}_{2}}$ : the thermodynamic factor $\gamma$, defined as $\gamma=\partial \ln \delta / \partial \ln P_{\mathrm{O}_{2}}$. In the simulations shown below, $\gamma$ spans values from -0.23 to 0.27 , as estimated from the nonstoichiometry information in Fig. C-2c.

Figure C-3a (middle and right columns) show the simulated behavior of $\hat{U}_{1,1}$ for the two models in Fig. C-4. As expected, these models predict exactly the same $1^{\text {st }}$ harmonic behavior after scaling. Indeed, any model for the reaction kinetics gives this same result, since the kinetics enter simply as a linearization of eqn. C-5. As such, comparison of the first harmonic (impedance) data in Fig. C-3a to the predicted $1^{\text {st }}$ harmonic tells us very little about the reaction kinetics - it merely confirms the kinetic limitation of the film.

In contrast, Figures C-3b and C-3c (middle column) show the simulated behavior of $\hat{U}_{2,2}$ and $\hat{U}_{3,3}$ when an energy barrier to dissociation is assumed to be limiting (model in Fig. C-4a). The calculated harmonics differ significantly from the data in several respects: magnitude, phase, and dependency on $P_{O 2}$. Attempts to better "fit" the data by adjusting $\gamma$ outside the range predicted by Fig. C-2c yield no better agreement. Thus the higher harmonics appear to be inconsistent with this mechanism. Several other models also yield poor agreement, suggesting that the rate limiting step is not any of the following: molecular (non-dissociative) adsorption, 
dissociation of other charged intermediates (e.g. peroxide), change in oxidation state of adsorbed monatomic intermediates, or exchange of oxygen vacancies between surface and bulk.

We were only able to find one mechanism that came close to explaining the higher harmonic data in Fig. C-3(b,c). Figures C-3b and C-3c (right column) show the predicted behavior of $\hat{U}_{2,2}$ and $\hat{U}_{3,3}$ when dissociative adsorption is assumed to be rate limiting (the mechanism illustrated in Fig. C-4b). In this case the value of $\gamma$ was adjusted manually to mimic the harmonic data, yielding values of $-0.27,-0.25$, and -0.23 for air, $5 \%$ oxygen, and $1 \%$ oxygen, respectively. The calculated spectra agree well with the data in Fig. C-3 in both magnitude and phase, including the counterclockwise phasor rotation and nullification of $\hat{U}_{2,2}$ near $5 \%$ oxygen (where $\gamma$ crosses $1 / 4)$. If correct, this match illustrates the extreme sensitivity of the harmonics to a very small $(16 \%)$ variation in a physical parameter $(\gamma)$.

To verify that these values of $\gamma$ are consistent with the thermodynamic properties of the film, we calculated the vacancy concentration $(\delta$ ) from $\gamma$ according to Faraday's law and the Nernst equation, which predict (see eqn. 5 of reference ${ }^{[45]}$ ):

$$
\delta=\frac{3 R T}{8 F^{2} c_{0} L} \frac{C_{\text {chem }}}{(-\gamma)}
$$

The results are shown as the data points in Fig. C-2c. Note that these data represent a point-bypoint calculation of $\delta$ based on the higher harmonics, while the line is a separate calculation based on a fit of $C_{c h e m}\left(P_{O 2}\right)$ to a particular nonstoichiometry model. The fact that these two calculations agree quantitatively supports the validity of the kinetic rate law. Furthermore, we show in reference ${ }^{[42]}$ that this same rate law is consistent with equilibrium exchange measurements on LSC over a wide range of $T, P_{O 2}$, and strontium doping.

\section{C-7. Discussion}

Since the late 1960's, linear impedance methods have proven to be one of the most useful techniques for studying solid-state ionic materials and devices ${ }^{[52]}$. Although the use of engineered electrode architectures dates to the same period ${ }^{[53]}$, it is only recently that welldefined thin films of mixed conductors have become available. By combining EIS with these films, workers have made significant contributions to our understanding of solid-state electrochemical reactions.

However, any macroscopic chemical or electrochemical rate measurement is an inherently indirect probe of mechanism, since it does not reveal the identity or concentrations of reactive intermediates. At best one can only disprove a particular mechanisms by showing that the measured rate is inconsistent with the rate law corresponding to that mechanism. By such process of elimination, it may be possible to reduce the number of candidate mechanisms. In catalysis this is often accomplished by operating a differential reactor far from equilibrium and examining reaction orders with respect to reactant or product concentrations. In electrochemistry the measurement of Tafel slopes provides similar information. 
This leads to the fundamental limitation of EIS (as well as other linearized rate methods). As shown in this paper, the linearized response (a Nyquist semicircle in the present case), is consistent with any rate law that predicts a linear force-flux relationship in the limit of equilibrium. Since a linear relationship is expected for any mechanism one wishes to consider, one can only judge mechanism based on scaling laws ( $R_{\text {chem }}$ vs. $P_{O 2}$ or $T$ ), which are inherently weak indicators of mechanism. Even with an overwhelming amount of equilibrium exchange data spanning $T, P_{O 2}$, and dopant concentration, uncertainties remain in the mechanistic interpretation $^{[42]}$.

In contrast, we have shown in this paper that by examining the higher harmonics, it is relatively straightforward with just a few measurements to eliminate candidate mechanisms based on inconsistency of the rate law with the nonlinear response. Furthermore, when one finds a mechanism that correctly predicts the measured rate law, the match involves a very high level of specificity to verifiable physical parameters. For example, in Fig. C-3 we saw that the phase rotation of the harmonics with $P_{O 2}$ is associated with a small (16\%) variation in the thermodynamic factor. That this small variation agrees very precisely with the known thermodynamics of the material provides a much higher level of confidence in the rate law than the activation energy, or the slope on a log-log plot of linearized rate vs. $P_{O 2}{ }^{[42]}$.

\section{C-8. Summary/Conclusions}

In the case of $\mathrm{La}_{0.6} \mathrm{Sr}_{0.4} \mathrm{CoO}_{3-\delta}$ on Gd-doped ceria, our results suggest that the rate-limiting step involves dissociative adsorption of $\mathrm{O}_{2}\left(\right.$ as $\left.\mathrm{O}^{2-}\right)$ on a limited number of vacant surface oxygen sites. This conclusion stands in contrast to the conventional wisdom, which usually assumes the existence of some type of energy barrier to charge-transfer. This assumption is usually based on the observation that the equilibrium exchange rate is activated. However, as shown in more detail elsewhere ${ }^{[42]}$, dissociative adsorption is also expected to exhibit a strong Arrhenius dependence due to the large negative enthalpy of dissociative adsorption, even when no energy barrier exists.

Application of the rate law measured here to studies of equilibrium $\mathrm{O}_{2}$ exchange on bulk samples of LSC yields very good agreement over a wide range of $T, P_{O 2}$, and $\mathrm{Sr}$ doping ${ }^{[42]}$. As shown below, these same kinetics also appear to explain the behavior of porous LSC electrodes. We speculate that part of the reasons LSC has a relatively low electrode impedance is that it has metallic band structure, which may help stabilize physisorbed $\mathrm{O}_{2}$ relative to p-type semiconducting materials (such as LSM or LSF). In addition, the very high vacancy concentration in LSC not only aids bulk transport, but also leads to faster kinetics through a very strong dependence of dissociative adsorption on surface vacancy concentration. The ideal cathode material would be a stable metallic oxide with very large numbers of free oxygen surface defects. Further studies are needed on various materials over a broader range of conditions to more fully understand these issues, and identify specific candidate materials that could improve SOFC cathode kinetics. 


\section{Parametric Studies of Porous LSC Electrodes}

\section{D-1. Introduction}

The mechanisms governing oxygen reduction in porous SOFC cathodes remains an active subject of debate despite a wealth of impedance measurements and modeling ${ }^{[9]}$. Fig. D-1 outlines some of the mechanisms either known or theorized in the literature to be important in determining the overall rate of the reaction ${ }^{[9]}$. Oxygen molecules are generally thought to adsorb somewhere onto one or more solid surface(s) where they undergo catalytic and/or electrocatalytic reduction steps to form partially reduced ionic/atomic species (sometimes called "electroactive species"). These species must transport along surfaces, or inside the bulk of the electrode to the electrolyte, where they are fully and formally incorporated as electrolytic $\mathrm{O}^{2-}$ ions. If, how, and where any of these processes happen, and what step(s) are rate determining for a particular electrode, is only partially understood.
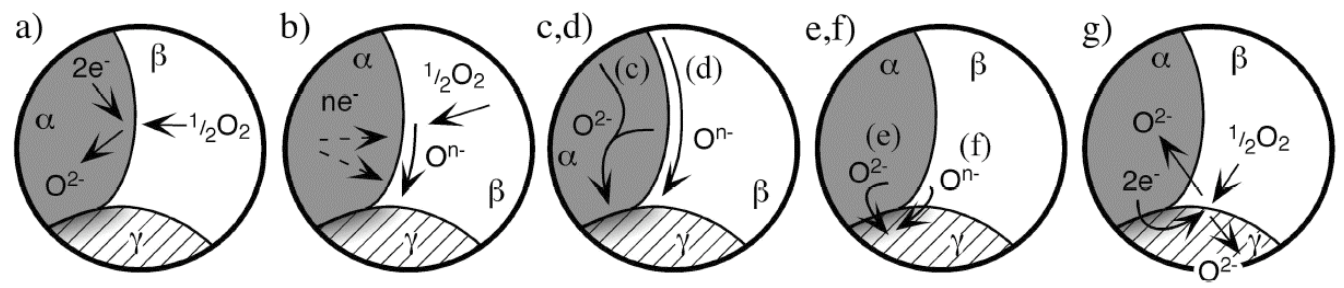

Figure D-1: Some of the physical processes thought to govern oxygen reduction in mixed-conducting SOFC cathodes. Phases $\alpha, \beta$, and $\gamma$ refer to the mixed-conductor, gas phase, and electrolyte, respectively. a) Incorporation of oxygen into the bulk of the mixed-conductor; b) Adsorption and/or partial reduction of oxygen on the surface of the mixed-conductor; c) Bulk or d) Surface transport of $\mathrm{O}^{2-}$ or $\mathrm{O}^{\mathrm{n}-}$, respectively to the $\alpha / \gamma$ interface, e) Electrochemical charge transfer of $\mathrm{O}^{2-}$ or $\mathrm{f}$ ) Combinations of $\mathrm{O}^{\text {n- }}$ and $\mathrm{e}^{-}$ respectively, across the $\alpha / \gamma$ interface, and $g$ ) rates of one or more of these processes wherein the electrolyte itself is active for generation and transport of electroactive oxygen species. Reproduced from reference ${ }^{[9]}$.

For mixed conductors with high oxygen vacancy concentration, and in the absence of interfacial change-transfer resistance (Fig 1-e,f), the impedance of a porous single-phase mixed conducting SOFC cathode often exhibits Gerischer-like behavior ${ }^{[9,10,24,54]}$, which appears on a Nyquist diagram as a half-tear-drop-shaped arc having a semi-circular shape at low frequency and a $45^{\circ}$ tail in the limit of high frequency ${ }^{[9]}$. This behavior is generally associated with colimitation by diffusion and reaction kinetics, coupled via a $1-D$ porous morphology ${ }^{[9]}$. However, many different mathematical models incorporating various combinations of the steps a-d in Fig. D-1 will, after linearization, predict this same basic Gerischer impedance response. This degeneracy among various models makes it impossible to judge from a Gerischer shape (alone) whether the dominant diffusion process is on the surface or in the bulk of the mixed conductor, or what kinetics are associated with oxygen ad- or ab- sorption steps. 
In order to gain further insight, workers have tried to fit measured EIS data to specific models over a wide rage of measurement conditions, testing the veracity of the model by comparing parameters extracted from the data to available values measured independently. For example, one model posed by Adler and coworkers ${ }^{[10,24]}$ hypothesized that under some conditions oxygen reduction will be dominated by a bulk transport pathway (steps a and c in Fig. D-1). In order to test this hypothesis, their model was linearized and solved to predict the impedance, and then fit to impedance data over a range of material compositions, morphologies, temperatures, and $P_{O 2}$. While the bulk diffusion and kinetic parameters extracted from the EIS data were found to agree with independent measurements under some conditions, it is hard to be definitive as to the completeness and generality of the model, given the tremendous uncertainties in comparing physical properties of bulk samples to those extracted from impedance data.

In the following, we extend the basic approach above by significantly broadening the range of processing and operating conditions - examining more closely the effects of substrate preparation, electrode composition ( $\mathrm{Sr}$ content), electrode firing temperature, testing conditions $\left(T, P_{O 2}\right)$, d.c. polarization, and time (degree of degradation). By augmenting these studies with NLEIS data, morphological characterization by BET and FIB-SEM, and select elemental analysis, we show that a consistent pattern begins to emerge that explains the majority of the data, and lights the way toward more specific and targeted future work.

\section{D-2. Fabrication of Porous LSC electrodes on SDC.}

Most of our studies of porous LSC electrodes were based on 20\% samaria-doped ceria (SDC). A green tape of 300 600 $\mu$ m thick SDC $\left(\mathrm{Ce}_{0.8} \mathrm{Sm}_{0.2} \mathrm{O}_{2-\delta}\right.$ plus $1 \% \mathrm{TiO}_{2}$ as a sintering aid, fabricated by Ceramatec, Inc., Salt Lake City) was cut into rectangular pieces of desired size. Usually several pieces (2 to 6) were heat-laminated together to from an electrolyte green body with desired thickness. Lamination was performed at $3000 \mathrm{psi}$ and about $70^{\circ} \mathrm{C}$ for $15 \mathrm{mins}$ with a carver laboratory press. Dense electrolyte was obtained without the need for organic solvent between lamination layers. The laminates were sintered at $1625-1650^{\circ} \mathrm{C}$ in air for 4 hours in a dedicated box furnace (Deltech Inc.) with ramp rate below $3^{\circ} \mathrm{C} / \mathrm{min}$ to form flat electrolyte plates. Well-used alumina setters (previously exposed to SDC) were employed to ensure flatness of the electrolyte plates, as needed for subsequent screen-printing. The shrinkage ratio of the SDC green tape was between $17.25 \sim 17.75 \%$ under the described conditions. The final sintered electrolyte plates typically had dimensions of $16 \mathrm{~mm} \times 20 \mathrm{~mm}$ for symmetric cells, and 1 inch $\times 0.75$ inch for micro-electrode half cells. SEM (FEI Sirion, UW) inspection and Archimedes density measurements were used to verify the fired electrolyte plates were uniformly dense with density higher than 96\% of theoretical. Except for the materials described in section D-8, below, no further surface treatment was performed - the materials were used net shape. EDX and XRD characterization (Philips 1820 XRD) of the green tape and sintered electrolyte showed a single $\mathrm{CeO}_{2}$-based phase. In order to achieve both good flatness and good mechanical strength, which are critical for sequential test cell fabrication and tests, the electrolyte plate thickness was kept between $0.5-1.0 \mathrm{~mm}$ for symmetric cells and $1.25-1.5 \mathrm{~mm}$ for half-cells.

Two compositions of LSC were studied: $\mathrm{La}_{0.8} \mathrm{Sr}_{0.2} \mathrm{CoO}_{3-\delta}$ (LSC-82) and $\mathrm{La}_{0.6} \mathrm{Sr}_{0.4} \mathrm{CoO}_{3-\delta}$ (LSC-64). LSC-82 and LSC-64 powders purchased from (Praxair specialty ceramics, and SCI engineering Co.) were processed into screen printing ink with a three roll mill. The powder was 
first hand mixed with organic vehicle, which is a mixture of binder, solvent, surfactant before roll milling. The ink vehicle contains $90-92 \mathrm{wt} \%$ alpha-terpineol, 8-10wt $\%$ Ethyl-cellulose, and $0.5 \mathrm{wt} \%$ oleic acid.

For porous LSC-82, both symmetric cells (LSC/SDC/LSC) and microelectrode half-cells (LSC/SDC) were used for comparison. For porous LSC-64, only half-cells were fabricated. For symmetric test cell fabrication, the finished electrode ink was screen-printed on both sides of the SDC electrolyte plates using a laboratory screen printer (HC-53, AMI) with 325/400 mesh count stainless steel screen (Sefar Printing Solutions, Inc). The dimensions of the printed electrode are $1.4 \mathrm{~cm} \mathrm{x} 1.4 \mathrm{~cm}$. $\left(\sim 2.0 \mathrm{~cm}^{2}\right)$. In order to guarantee the symmetric nature of the electrodes, these two electrodes were screen printed sequentially and fired at the same time. After printing of each side, the electrolyte plate was heated on a hot plate in a hood at $125-150^{\circ} \mathrm{C}$ for $10-15$ minutes to evaporate volatile solvent in the slurry and solidify the printed electrode layer. Because the presence of the organic binder, the solidified electrode wouldn't be deformed or damaged in the second printing, during which the first printed electrode had direct contact with the substrate holder. The printed symmetric cells were then sintered at different temperatures from $900^{\circ} \mathrm{C}$ to $1100^{\circ} \mathrm{C}$ for 4 hours in stagnant room air using a Lindberg/Blue tube furnace. The ramping rate for sintering process is $3^{\circ} \mathrm{C} / \mathrm{min}$ upward and $4-5^{\circ} \mathrm{C} / \mathrm{min}$ downward.

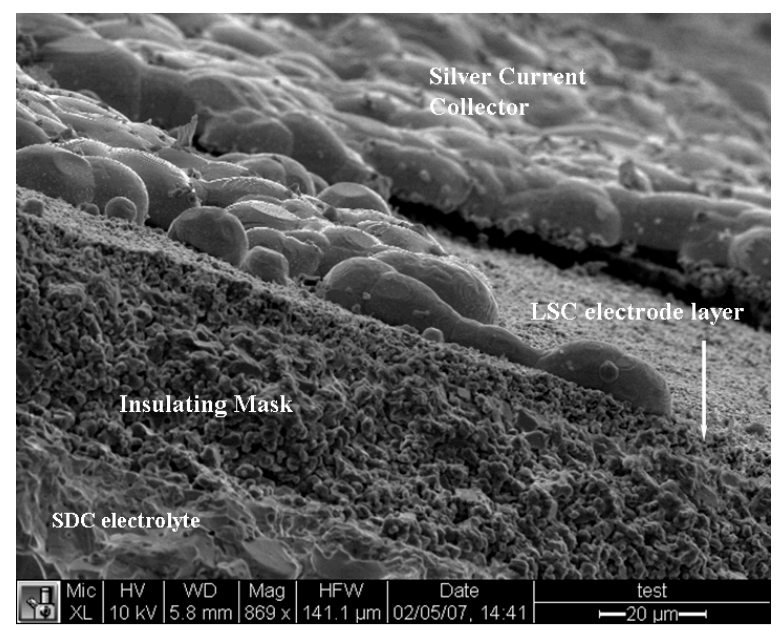

Figure D-2. Cross sectional image of a fabricated half-cell, incorporating an improved $\mathrm{MgO} /$ spinel mask layer.

For microelectrode half-cells, the same material was used for the working electrode (WE), the reference electrode (RE) and the counter electrode (CE). Half-cell fabrication follows the same procedure described in Section A using pattern B design for LSC electrode materials. A single insulating mask layer of $\mathrm{MgO} /$ spinel was printed on the sintered SDC electrolyte plate and sintered at $1375-1400^{\circ} \mathrm{C}$ for $4 \mathrm{hrs}$. The printing quality was visually inspected with a lab microscope before electrode printing. As shown in Section A, the sintered mask is about 15$20 \mu \mathrm{m}$ thick with good coverage. The working electrode and reference electrode were then screen-printed on top of the mask layer. After the counter electrode was printed (or brush 
painted in some cases) on the back side of the electrolyte plate, the test cells were fired at various temperatures from $900-1150^{\circ} \mathrm{C}$ for $4 \mathrm{hrs}$.

Some LSC-64 half-cells were also fabricated on thick cylindrical pellets of GDC. In these LSC-64 cathode layers were fabricated by screenprinting and firing LSC-64 ink onto pre-fired $\mathrm{Gd}_{0.1} \mathrm{Ce}_{0.9} \mathrm{O}_{1.95}$ (GDC-10) pellets. The pellets were fabricated by pressing GDC-10 powder (surface area: $36.5 \mathrm{~m} 2 / \mathrm{g}$ ) from Fuel Cell Materials with a $1.535 \mathrm{~cm}$ diameter pellet die. The green GDC-pellets were then fired at $1350 \mathrm{C}$ for 4 hours in air. LSC-64 electrodes of area 0.28

$\mathrm{cm}^{2}$ were then screen printed on both sides of the dense electrolyte and a LSC-64 reference electrode was applied to the circumference of the pellet. The cell was then fired in air at $1100^{\circ} \mathrm{C}$ for 2 hours. Gold mesh current collectors were applied to the working and counter electrodes to ensure contact and current distribution over the entire face of the electrodes, and a gold wire spanned the circumference of the reference electrode.

\section{D-3. Microstructural Characterization.}

In order to link microstructure to electrode performance, the morphology of electrodes sintered at different temperatures was investigated using scanning electron microscopy (SEM). Figure D-3 and D-4 show typical images of the cross section and porous structure of LSC-82 and LSC-64 electrodes sintered at different temperature on SDC electrolyte. Generally speaking, the average thickness of the sintered electrode decreased with increasing sintering temperature, ranging from 4 to $8 \mu \mathrm{m}$. Given consistent screen-printing thickness and quality, these changes were expected because of enhanced densification at higher sintering temperature. Sintering temperature effects are also visible at the electrode/electrolyte interface. The LSC-82 electrodes fired at high temperature $\left(1050^{\circ} \mathrm{C}\right.$ and $\left.1100^{\circ} \mathrm{C}\right)$ show much better overall bonding quality to the electrolyte than the electrode fired at lower temperature $\left(900^{\circ} \mathrm{C}\right)$. At certain positions the electrode fired at $900^{\circ} \mathrm{C}$ seems only loosely attached to the electrolyte at several locations while most of the electrolyte area were not physically bonded (Figure D-3(a)). Our observation showed that the electrode sintered at $900^{\circ} \mathrm{C}$ was very easy to be scratched off with bare fingers. This also points out a poorly bonded electrode/electrolyte interface and loosely connected porous structure. On the other hand, only small differences in the porous structure can be discerned visually between samples sintered at different temperatures. While the sizes of electrode cluster and connected particles grew slightly larger with increasing sintering temperature, it is hard to tell the difference of overall porosity and pore size distribution. Generally, the majority pore size ranges between 0.2 to $0.8 \mu \mathrm{m}$ for all sintering temperature with maximum pore size ranging between 1.2 to $2 \mu \mathrm{m}$. LSC-64 electrodes sintered at different temperatures showed similar trends. 
Figure D-3. SEM images of LSC82 electrodes sintered at $900-1100^{\circ} \mathrm{C}$

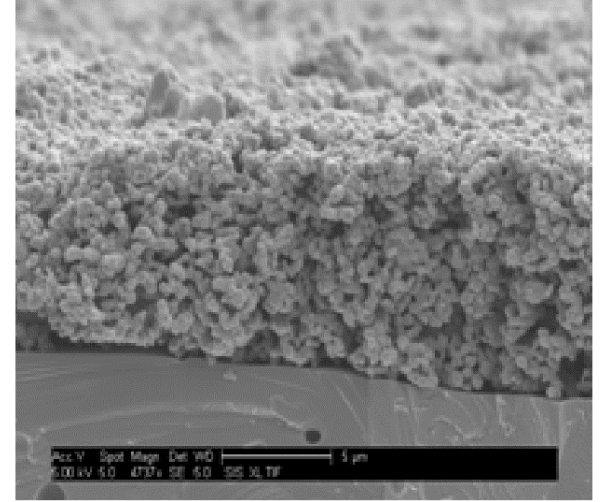

LSC8210/SDC interface sintered at $900^{\circ} \mathrm{C}$

a)

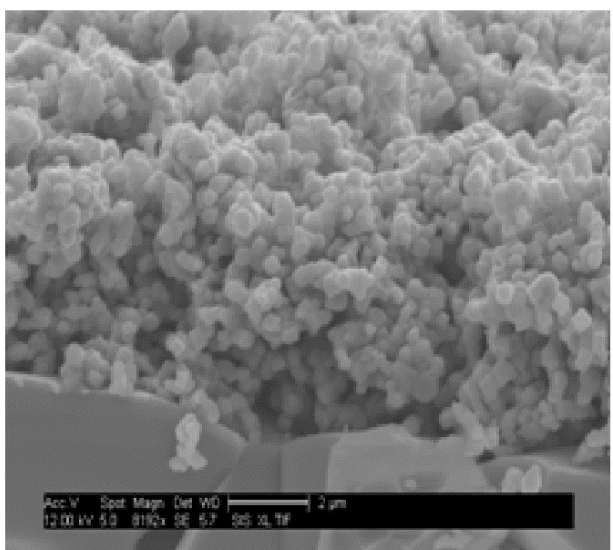

LSC8210/SDC interface sintered at

b)

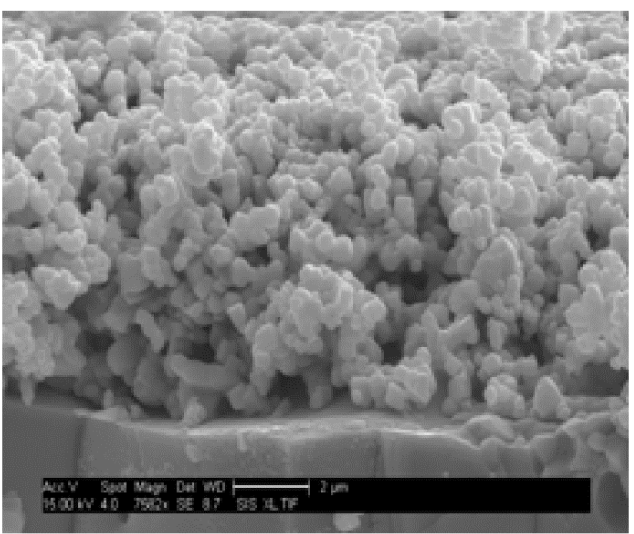

LSC8210/SDC interface sintered

c) at $1000^{\circ} \mathrm{C}$

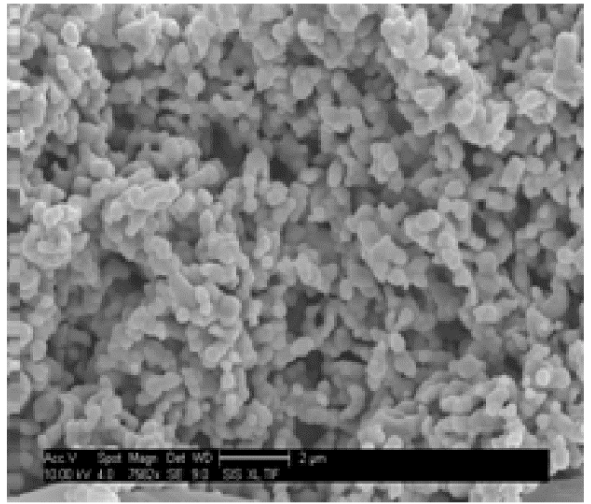

Porous Structure of LSC8210 electrode sintered at $900^{\circ} \mathrm{C}$

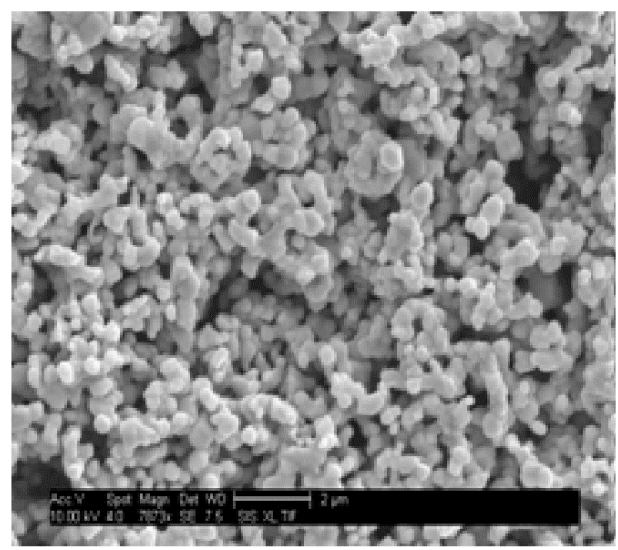

Porous Structure of LSC8210 electrode sintered at $950^{\circ} \mathrm{C}$

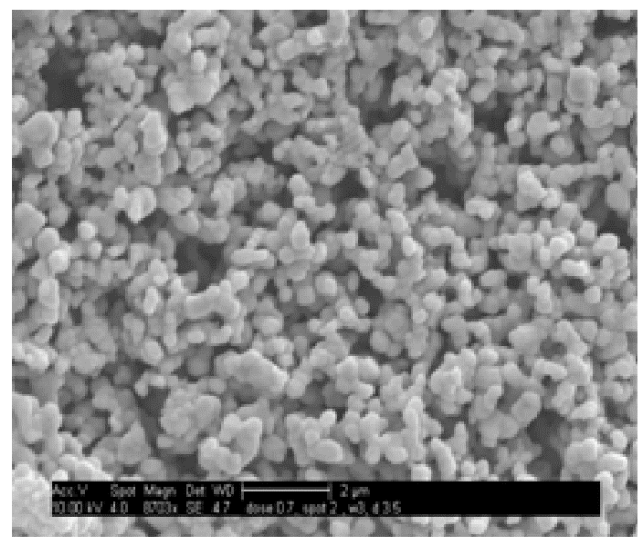

Porous structure of LSC8210 electrode sintered at $1000^{\circ} \mathrm{C}$ 


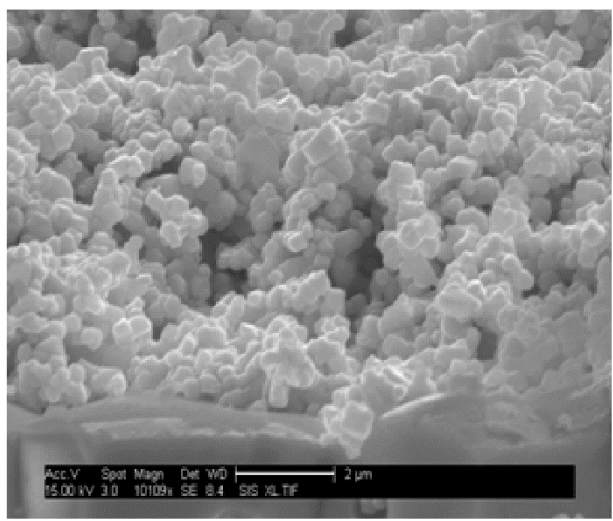

LSC8210/SDC interface sintered at $1050^{\circ} \mathrm{C}$

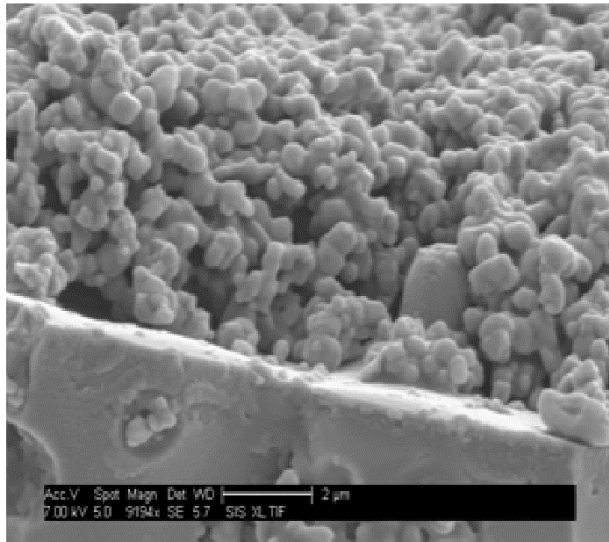

LSC8210/SDC interface sintered at

e) $1100^{\circ} \mathrm{C}$

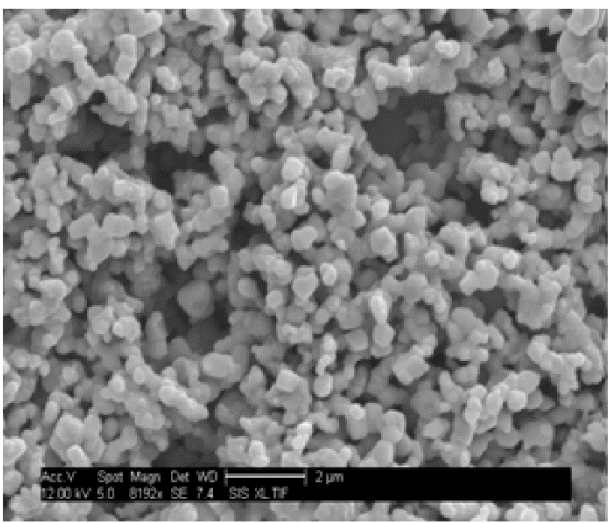

Porous structure of LSC8210 electrode sintered at $1050^{\circ} \mathrm{C}$

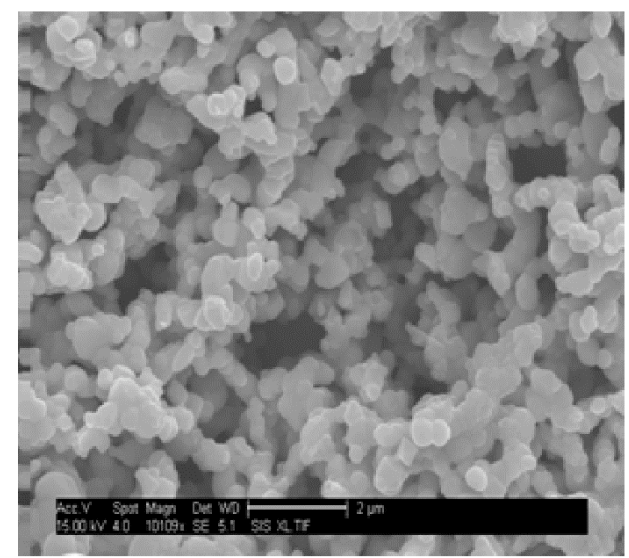

Porous Structure of LSC8210 electrode sintered at $1100^{\circ} \mathrm{C}$ 
Figure D-4. SEM images of LSC64 electrodes sintered at $1000-1150^{\circ} \mathrm{C}$

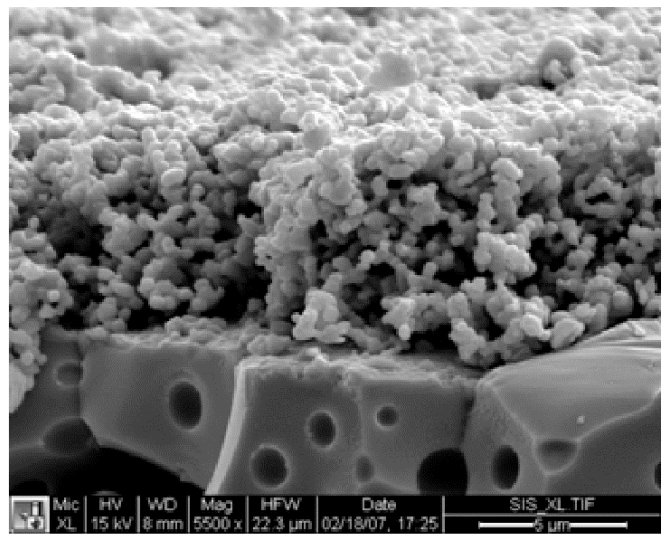

a)

LSC64 WE sintered at $1000^{\circ} \mathrm{C}$

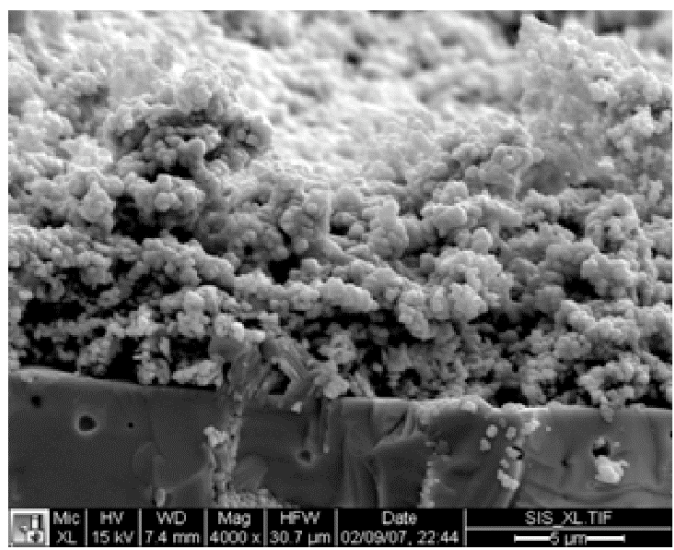

b)

LSC64 WE sintered at $1100^{\circ} \mathrm{C}$

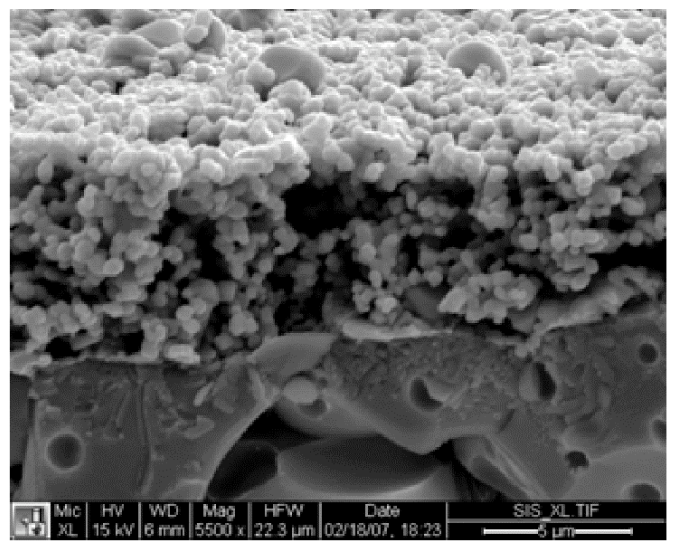

LSC64 WE sintered at $1050^{\circ} \mathrm{C}$

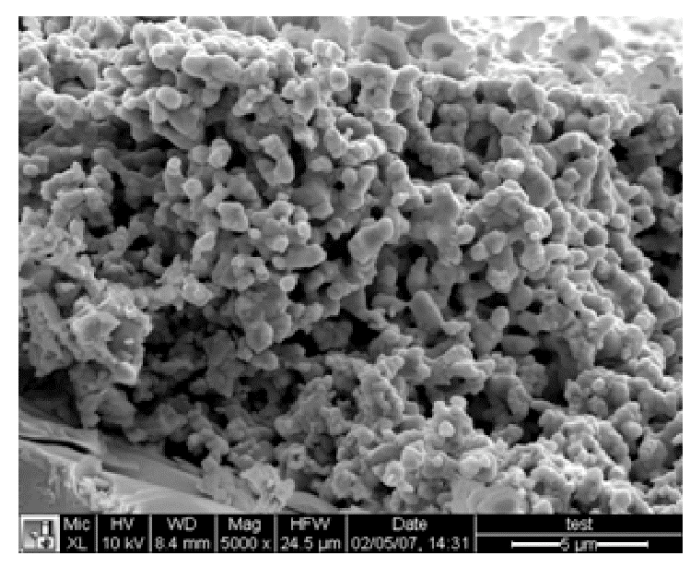

LSC64 WE sintered at $1150^{\circ} \mathrm{C}$ 
Through a NSF-funded collaboration with Northswestern University and the University of Michigan, two LSC-82 electrodes fired at $950^{\circ} \mathrm{C}$ and $1100^{\circ} \mathrm{C}$ were also examined with FIB-SEM, a technique used to generate a $3 \mathrm{D}$ images of the microstructure based on serial sectioning by Focused Ion Beam (FIB) milling ${ }^{[55]}$. For example, Fig. D-5 shows a 3D image of one of the samples.

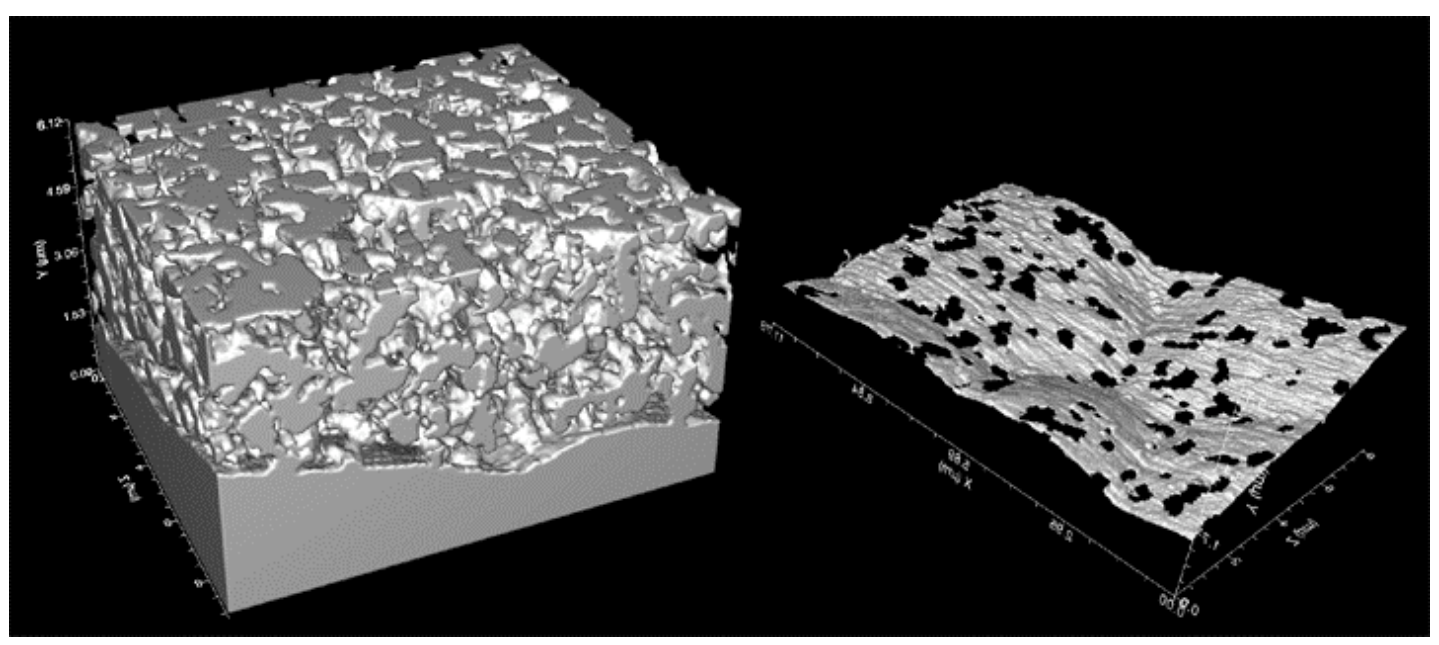

Fig. D-5. (a) 3D reconstruction based on FIB-SEM serial sectioning of a porous LSC cathode on a SDC electrolyte, fired at $1100^{\circ} \mathrm{C}$ and (b) the interface between LSC and SDC (the black holes represent the LSC/GDC contact areas).

The benefit of this technique is that once generated, the image can be mined for microstructural parameters not available from 2D images alone. Table D-1 summarizes some of the microstructural data obtained for these two samples. If correct, these images suggest that firing at $950^{\circ} \mathrm{C}$ vs. $1100^{\circ} \mathrm{C}$ has little influence on the microstructural parameters relevant for electrochemical activity (surface area, bonding, tortuosity). This may be a consequence of the rather course powders used for these electrodes. Once a sufficient temperature is reached to achieve particle necking and bonding to the electrolyte, further coarsening and densification is rather slow. However, some care must be taken not to over-interpret this data - although the analysis is quantitative, these images only capture a small section of the electrode material.

Table D-1. Three-dimensional microstructural parameters for LSC-82 cathodes sintered at $950^{\circ} \mathrm{C}$ and $1100^{\circ} \mathrm{C}$ extracted from FIB-SEM.

\begin{tabular}{|l|c|c|c|c|c|}
\hline $\begin{array}{l}\text { Firing } \\
\text { Temp. }\end{array}$ & Porosity & $\begin{array}{c}\text { Volumetric } \\
\text { Surface Area }\end{array}$ & $\begin{array}{c}\text { Fraction of } \\
\text { Electrolyte } \\
\text { Bonded to LSC }\end{array}$ & $\begin{array}{c}\text { Tortuosity for } \\
\text { Bulk Ion } \\
\text { Transport in LSC }\end{array}$ & $\begin{array}{c}\text { Tortuosity for } \\
\text { Gas-Phase Mass } \\
\text { Transfer }\end{array}$ \\
\hline $950^{\circ} \mathrm{C}$ & $42 \%$ & $2.7 \mu \mathrm{m}^{-1}$ & $22 \%$ & 1.4 & 1.6 \\
\hline $1100^{\circ} \mathrm{C}$ & $46 \%$ & $2.8 \mu \mathrm{m}^{-1}$ & $21 \%$ & 1.3 & 1.7 \\
\hline
\end{tabular}


In order to confirm that the material only coarsens slowly, the microstructure of sintered electrode inks was also characterized by BET surface area measurements. Unfortunately the electrodes themselves lack sufficient material to get an accurate BET measurement. Thus the same screen printing ink used in cell fabrication (2 3 grams) was fired in alumina containers under the same sintering conditions and furnace as the electrodes. The resulting sintered mass of porous LSC was then lightly crushed into small pieces to fit into a BET test tube (we do not believe this step changed the surface area significantly). Some of the fired inks were also kept at $750^{\circ} \mathrm{C}$ (the highest testing temperature for electrochemical measurements) for $4 \sim 6$ days to detect any possible microstructural evolution of the electrode under testing conditions.

Figure D-6 shows the relationship between firing temperature and electrode surface area of LSC-82 and LSC-64 electrode materials. The materials clearly exhibited measurable densification between $1000^{\circ} \mathrm{C}$ and $1200^{\circ} \mathrm{C}$. However, between $950^{\circ} \mathrm{C}$ and $1100^{\circ} \mathrm{C}$, the surface area of LSC-82 only changes from about $1.7 \mathrm{~m}^{2} / \mathrm{g}$ to $1.2 \mathrm{~m}^{2} / \mathrm{g}$. Also, assuming a porosity of $45 \%$, these surface areas correspond to volumetric surface areas of $\sim 6.3 \mu \mathrm{m}^{-1}$ and $4.4 \mu \mathrm{m}^{-1}$, respectively. These values are about twice as high as those extracted from FIB-SEM, suggesting that the un-printed ink may not be as fully necked as the printed electrodes in this same range of firing temperatures. In other words, the observed change in BET surface area probably represents an upper estimate of the degree of coarsening. Taken together with the FIB-SEM results, it would appear that the electrode surface area of LSC-82 probably changes less than $\sim 50 \%$ between firing temps of 950 and $1100^{\circ} \mathrm{C}$.

The BET results shown no detectable densification over $4 \sim 6$ days at operating temperature $\left(750^{\circ} \mathrm{C}\right)$ for the electrodes fired at $900^{\circ} \mathrm{C}$ and $1000^{\circ} \mathrm{C}$. Therefore, we believe that the electrode microstructure itself is stable under test conditions.

\section{Sintered Electrode Surface Area}

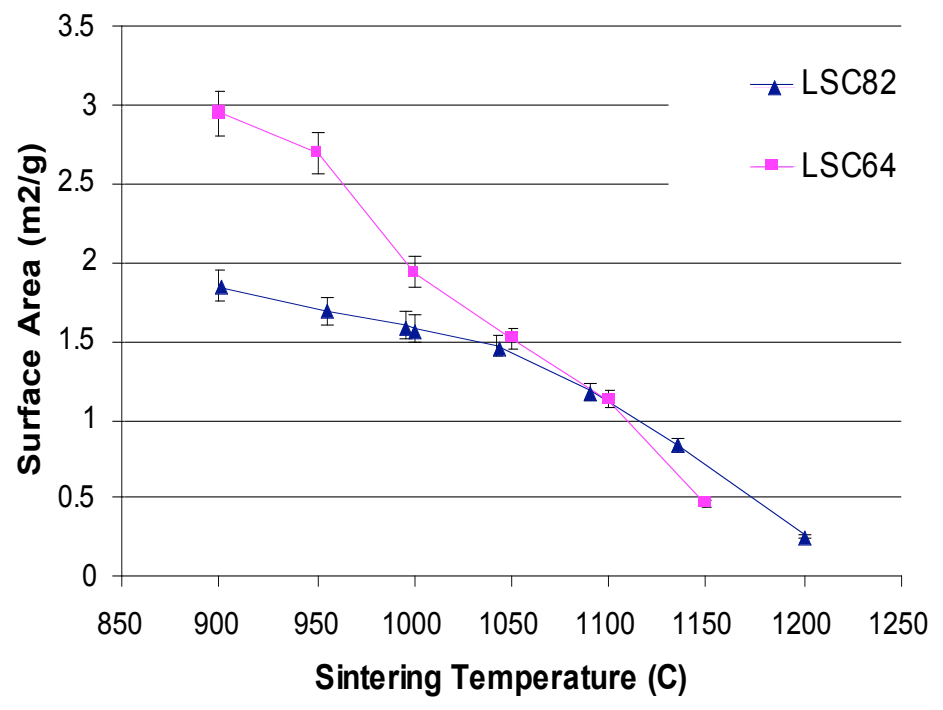

Figure D-6. Surface areas of sintered LSC powders measured by BET 


\section{D-4. Elemental Analysis.}

Surface contaminants, or deviations of surface composition from the bulk could have a strong possible influence on the catalytic behavior of $\operatorname{LSC}^{[9]}$. In this study, the surface compositions of as-sintered (S) and experimentally tested (T) electrodes were studied by energy dispersion X-ray spectroscopy (EDX/EDS). Surface compositions were analyzed at randomly chosen locations (3-5 positions) from both top surface and the cross section of fractured test cells, and then the results compared.

Unfortunately EDX did not reveal a detectable pattern of compositional variation in La, $\mathrm{Sr}$, Co above the noise. However, we did notice that small amounts of $\mathrm{Na}, \mathrm{Bi}$, and $\mathrm{Cr}$ appear following testing. We believe the Na results from inorganic binders present in the test furnace materials. $\mathrm{Bi}$ is present in the Pt ink used in fabrication of the oxygen sensor that is inserted into the test furnace. Finally, Cr may come from the alloy sheath material of the thermocouple in the test furnace, which is partially exposed via the open bottom end of a thermo-well (see below).

\section{D-5. Electrochemical Testing.}

Several high-throughput test stations for simultaneous study of up to six symmetric cells (or three half cells) were built in our laboratory for this project. A schematic of one such test station is shown in Fig. D-7. The station consists of a vertical orientated compact tube furnace, a dense high purity alumina chamber tube (99.8\% alumina, Coorstek Co.) with one close end, and a home-made test stand. The test stand is constructed with high purity alumina tubes, an alumina holder plate (Ceramatec, Inc), wiring components (silver wire, 99.5\%, Alfa Aesar), and high purity alumina fiber insulating materials. This test stand structure is fixed to the gas tight alumina chamber tube via a stainless steel top plate and a flange by bolts and nuts to form gas tight enclosure. The top plate also houses the gas inlet and outlet fittings, thermal couple and gas sensor throughputs, and electrical connections throughputs. The thermal couple and gas sensor throughputs were sealed by Swagelok Vacuum Fitting. The Electrical connections throughputs were sealed by Epoxy glue. The stainless steel flange, which was glued to the gas tight alumina tube with alumina based ceramic glue, has an O-ring groove to achieve gas tight seal between the steel top plate and the alumina tube. The viton o-ring used can sustain long-term operating temperature up to $200^{\circ} \mathrm{C}$. A high flow rate fan was used to cool the top plate and flange during tests. The gas tight feature of this setup has been experimentally tested under temperature up to $900^{\circ} \mathrm{C}$ and various gas conditions down to $500 \mathrm{ppm}$ of oxygen in nitrogen. The entire assembly is placed in a non-inductively wound Mellon furnace element, wrapped in fibrous insulation, and held in a 5 gallon paint can (Ceramatec, Inc.).

In order to control and monitor the experimental conditions, type-K thermocouples and oxygen gas sensors were placed to close vicinity of the test cells. The thermocouples (from Omega Engineering Co.) are built with high quality Inconel and Super Omegclad XL ${ }^{\circledR}$ alloy sheath to minimize corrosion under highly oxidant condition and gas phase impurity contamination. The internal oxygen gas sensor is home made with a gas tight dense YSZ electrolyte tube (Mcdanel Advance Ceramic Technologies, LLC), platinum wires (Alfa Aesar) and platinum ink (Engelhard Co.). Porous platinum electrodes were fabricated on both sides of the closed end of the YSZ tube by brush-painting of platinum ink and sintering at $1050^{\circ} \mathrm{C}$ for 4hrs. During operation, the platinum electrode on the outside surface of the closed end was 
exposed to controlled gas inside the alumina test tube while the inside platinum electrode was exposed to room air through the tube opening. The potential difference between the platinum electrodes was used to determine the oxygen concentration near the test cells (potentiostatic mode). During operation, continuous gas flow between $(50-150 \mathrm{sccm} / \mathrm{min})$ is introduced to the bottom of the alumina enclosure tube through the central gas inlet tube inside the test stand structure for gas concentration control. The gas is heated up to the test temperature during the downwards flow in order not to create temperature disturbance in side the test chamber. For $100 \% \mathrm{O}_{2}$ and $5 \% \mathrm{O}_{2}$, gas flow rate of $100 \mathrm{sccm} / \mathrm{min}$ is good enough to ensure steady gas phase concentration. For $1 \% \mathrm{O}_{2}$ and below, the gas flow rate needs to be increased to 150 or 200 $\mathrm{sccm} / \mathrm{min}$ to overcome the air diffusion from the top plate.
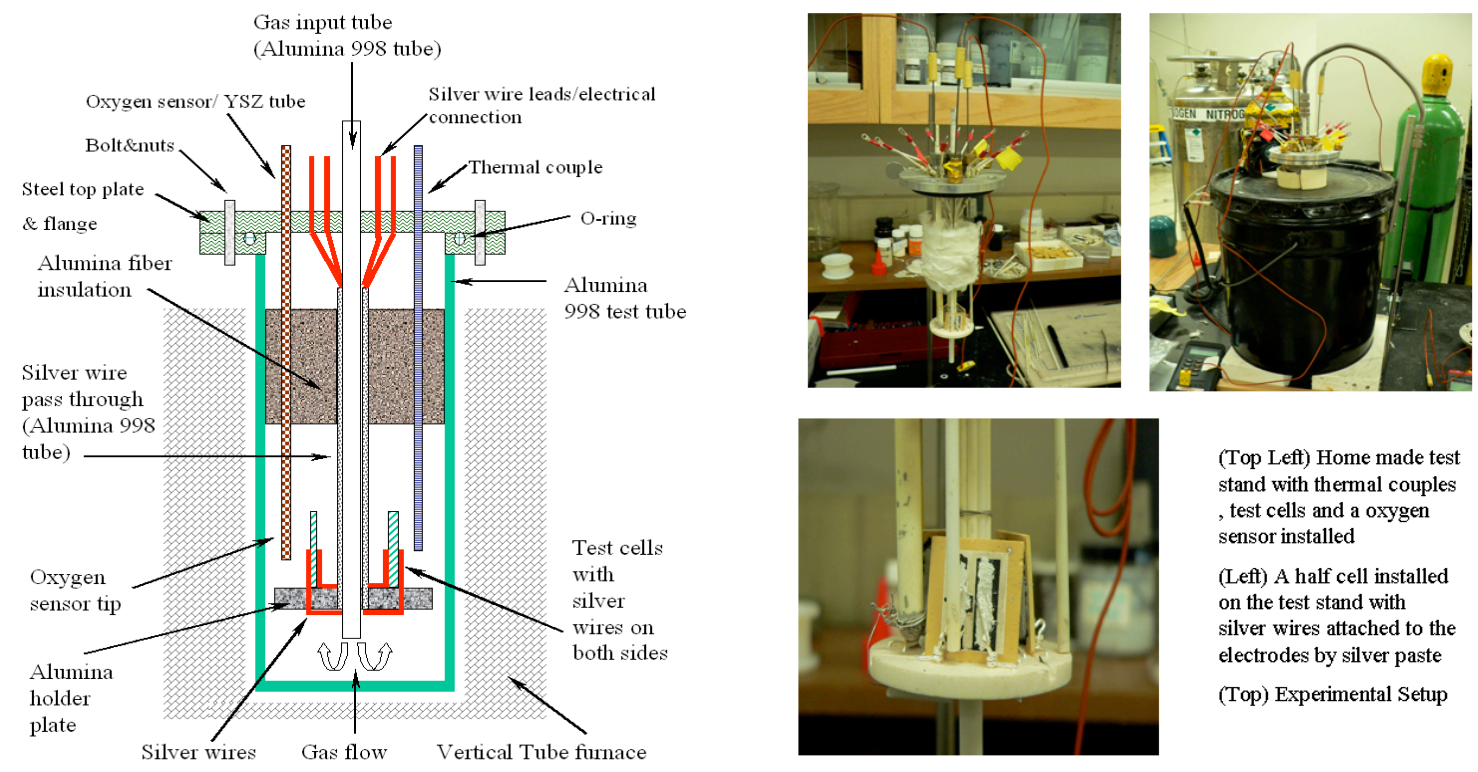

(Top Left) Home made test stand with thermal couples , test cells and a oxygen sensor installed

(Left) A half cell installed on the test stand with silver wires attached to the electrodes by silver paste

(Top) Experimental Setup

Figure D-7. Schematic (left) and photos (right) of one of the high-throughput test stations used for electrochemical measurements.

All electric connections on the test stand were built with pure silver wires $(1 \mathrm{~mm}$ in diameter), which have long term working temperature up to $900^{\circ} \mathrm{C}$ with good mechanical strength and electric conductivity. Each silver wire was separated and insulated by alumina tubes on the test stand. These wires went through holes on the holder plate to make connection with test cells. Silver paste (Ceramatec Inc) is used to make electric connections between the test cells and silver wires on the test stand. In the case of the symmetric cells, the entire electrode was coated with silver paste as a current collector. In the case of the half-cells, the silver paste only made contact with the LSC bus, located several $\mathrm{mm}$ from the active working electrode. While silver wires mechanically held test cells on the test stand in vertical orientation, the silver paste glued the silver wires to the electrode surfaces to make electrical connections. After assembly at room temperature, the furnace temperature was raised to $750^{\circ} \mathrm{C}$ at ramp rate about $2-3^{\circ} \mathrm{C} / \mathrm{min}$. The silver paste was well sintered to form solid electric and mechanical connection between the test cells and the silver. Because silver has much larger thermal expansion coefficient (TEC) than 
perovskite electrode materials like LSC, ramp rates slower than $2^{\circ} \mathrm{C} / \mathrm{min}$ were used for any temperature transition during our tests to prevent current collector delamination. Our observation after cell tests showed that the sintered silver current collector was firmly attached to the LSC electrode surface mechanically. The physical bonding positions are uniformly distributed all over the contact area (Figure D-8). The sintered silver layer is averagely about $20-30 \mu \mathrm{m}$ thick over the electrode surface. This thickness could increase to $0.05 \sim 0.1 \mathrm{~mm}$ around the supporting silver wires.
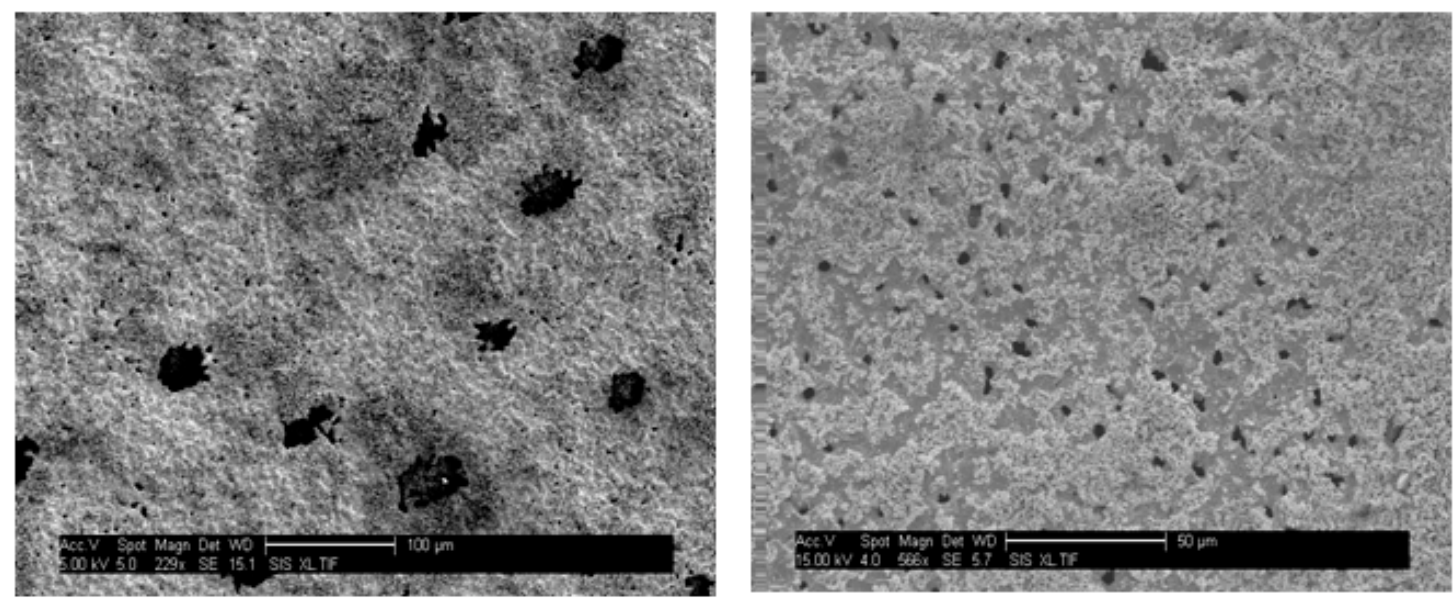

Figure D-8. Contact interface between Ag current collectors and LSC electrodes

Two or three test cells with same LSC electrode material were studied simultaneously in the same test furnace to ensure the identical thermal and gas phase conditions. LSC-82 electrodes were mostly studied using symmetric cells when the half cell design was still under development. LSC-64 electrodes were studied using the half cell design. Both electrochemical impedance and DC polarization measurements were carried out using a Solartron 1287 potentiostat and a 1252A frequency response analyzer. For symmetric cells, a symmetrical twoelectrode set-up was used with one silver wire connected to each electrode. For half-cells, a three terminal configuration was used, in which the working electrode, reference electrode and counter electrode have their own separate connection wire. Some additional measurements were also made using NLEIS, as described in Section B.

EIS measurements were made mostly at open circuit conditions in the frequency range of $0.25 \mathrm{~Hz}-300 \mathrm{kHz}$ in $\mathrm{O}_{2} / \mathrm{N}_{2}$ blended gases with oxygen content of $100 \%, 21 \%$ (Air), $5 \%$ and $1 \%$. All the gases were certified industrial gases purchased from different gas vendors. Mass flow controllers for $\mathrm{N}_{2} / \mathrm{O}_{2}$ blended gases controlled the gas flow rate to the gas inlet tube (100-200 $\mathrm{sccm} / \mathrm{min})$. The measurements were performed potentiodynamically at temperatures from $600^{\circ} \mathrm{C} \sim 750^{\circ} \mathrm{C}$ with decreasing perturbation frequency (10 15 frequency points per decade). Depending on the electrolyte thickness, temperature and gas condition, the voltage perturbation amplitude applied (over the whole test cell for symmetric cells, and between the WE and RE for half cells) was adjusted between $10 \mathrm{mV}$ RMS and 40mv RMS, which was equivalent to $5-10 \mathrm{mV}$ RMS on the working electrode. Ideally, EIS measurements should be made under stationary 
conditions. Thus, after each change of temperature or atmosphere, the cells were kept at their new conditions until they showed repeatable impedance data within a time window of several hours. Usually the impedance spectra were collected at least three times over a time frame of $8 \sim 12$ hours at each temperature or gas conditions to see if severe degradation or change existed. In the case of continuous change, more data were collected over time frames ranging from 2 to 20 days in order to track the degradation process.

For half cell cells (only), cyclic voltammagrams (CV) study were performed occasionally in the voltage range (between $\mathrm{WE}$ and $\mathrm{RE}$ ) from $-0.5 \mathrm{~V}$ to $0.1 \mathrm{~V}$ with $1 \sim 2 \mathrm{mV} / \mathrm{S}$ sweep rate to study the electrode behavior under polarization. Long term (1-2 months) dc potentiostatic polarization was also carried out at $750^{\circ} \mathrm{C}$ in dry air to study the stability and degradation behavior of LSC electrode materials. The polarization voltage (overpotential) on the working electrode was controlled between $125 \mathrm{mV}$ and $150 \mathrm{mV}$ during dc measurements. The dc polarization was interrupted briefly every 3 or 4 days for impedance measurement (15-30mins), which was carried out at open circuit condition. After each impedance study, the polarization study was resumed and the WE polarization voltage was adjusted according to the just measured impedance spectra.

\section{D-6. Post-Experimental Data Analysis}

Typical impedance spectra for symmetrical LSC cells are shown in Fig. D-9. In general we found that electrodes fired below $950^{\circ} \mathrm{C}$ had two primary impedance features - one below $\sim 100$ $\mathrm{Hz}$, and the other in the $\mathrm{kHz}$ range. Increased firing temperatures led to disappearance of the high frequency arc. As discussed more fully in Section D-8, below, we believe the high frequency arc is related to interfacial ion transfer between LSC and SDC when the electrode/electrolyte bonding is inadequate. As the firing temperature is further increased, the characteristic frequency of the remaining arc decreases, while the impedance magnitude increases.

Because of great uncertainty (and variability) in the shape of the impedance, we did not fit the data to an equivalent circuit. Instead, we attempted to subtract any interfacial effects from the data as depicted in Fig. D-9 (right). In some cases we used the method of Berthier ${ }^{[56-58]}$. In others we fit the high-frequency data (only) to a Randles circuit (with $Z_{F}=R_{t}+$ a semi-infinite Warburg impedance) to get values for inductance, double layer capacitance, and interfacial resistance, and then subtracted these from the data ${ }^{[9]}$. At electrode firing temperatures above $\sim 950^{\circ} \mathrm{C}$ the interfacial effects were small, and so usually no such subtraction was required in order to further analyze the low frequency data.

The remaining data (consisting primarily of a single asymmetrical low-frequency arc) was then characterized in terms of a characteristic time constant $t_{0}$ (defined as the reciprocal of the peak frequency (in radians/s) where the data has maximum imaginary value), and a characteristic resistance $R_{0}$ (defined as the imaginary magnitude at the peak frequency). The benefit of this approach is that it captures the gross scaling of the impedance frequency and magnitude without making any detailed judgments regarding the shape (which is often subject to dispersion or distortion by artifacts of little fundamental significance). The remaining data analysis thus focused on how $R_{0}$ and $t_{0}$ scale with the processing and operating conditions of the electrode (rather than on the specific shape of the impedance). 

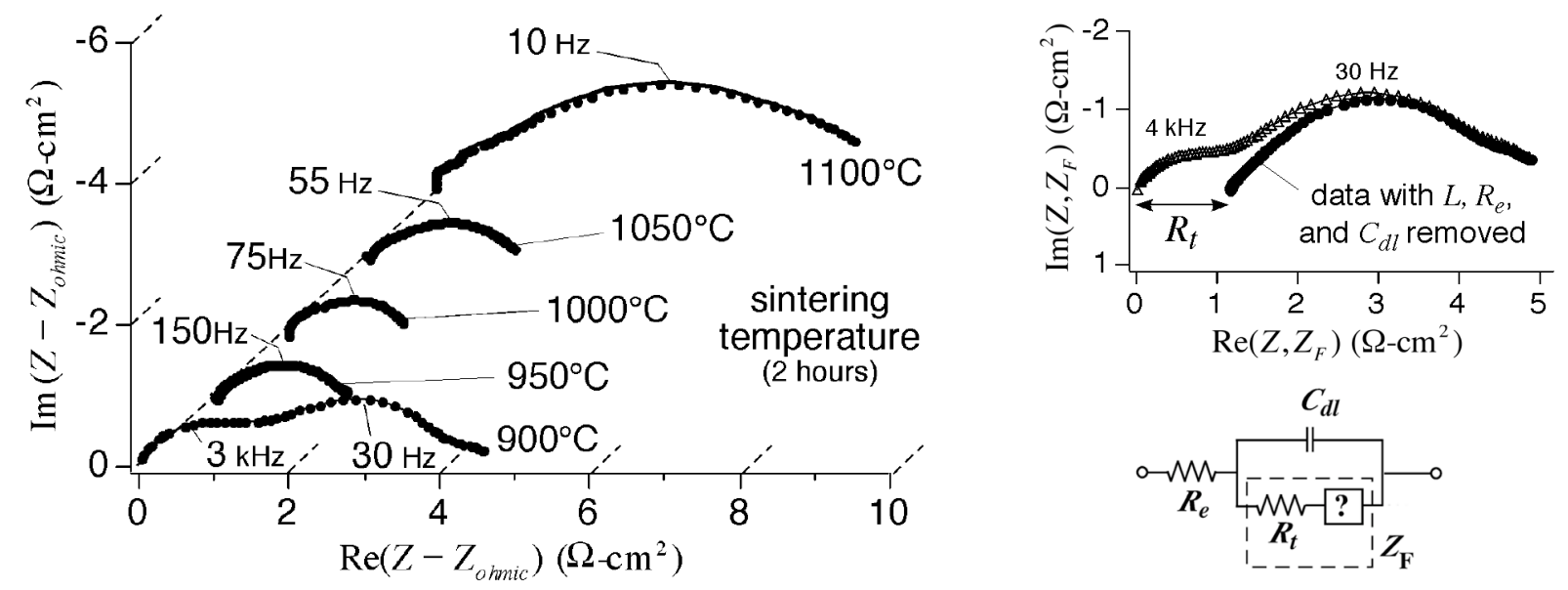

Fig. D-9. Measured impedance of porous LSC-82 symmetric cells at $750^{\circ} \mathrm{C}$ in air. Left: Dependence of impedance on electrode firing temperature. Right: Application of a Randles circuit to remove series inductance, double-layer capacitance, and interfacial charge-transfer resistance.

\section{D-7. Evidence for Co-limitation and Dissociative Adsorption}

As exemplified by the data in Figure D-9, the low frequency arc appearing in the impedance data was generally asymmetrical, and (after removal of high-frequency effects) generally had a "Gerischer-like" shape. As discussed in Reference ${ }^{[9]}$, a Gerischer impedance is expected in any porous electrode where the overall reaction is limited by both kinetics (in this case $\mathrm{O}_{2}$ reduction on LSC) and a cumulative transport process (in this case diffusion of oxygen intermediates in (or on) the LSC phase to the electrolyte). As long as the utilization region (active region of the electrode) is smaller than the electrode thickness, but larger than individual electrode particles, a Gerischer impedance would be expected.

Unfortunately the data we obtained only sometimes fits a Gersicher impedance, and often not very well. This could mean that the electrode is not operating in a co-limited regime. Another possibility, however, is that the material is not very homogeneous, either in morphology, material properties, lateral current distribution, electrode/electrolyte contact, etc. These factors could lead to distortions of the impedance from a Gerischer shape, even though the underlying physics is still co-limiting. If such distortions were present, it would make detailed fitting of individual spectra to a model very difficult. However, an alterative approach is to examine how the gross scaling of the arc $\left(R_{c}\right.$ and $\left.t_{c}\right)$ change with the various experimental parameters, and then compare these trends to those expected from a model. 
As described elsewhere ${ }^{[9,10,24]}$, the gross scaling of the impedance in the case of co-limited behavior in LSC can be summarized as:

$$
\begin{gathered}
t_{c}=\frac{4 \delta(1-\varepsilon)(-\gamma)}{a \Re_{0} V_{L}}, \\
R_{c}=0.35\left(\frac{R T}{2 F^{2}}\right) \sqrt{\frac{V_{L}}{(1-\varepsilon) \delta D_{v}^{e f f} a \Re_{0}}},
\end{gathered}
$$

where $\delta$ is oxygen nonstoichiometry, $\varepsilon$ is porosity, $a$ is volumetric surface area, $V_{L}$ is the pseudo-cubic lattice volume, $\gamma=\partial \ln \delta / \partial \ln P_{O_{2}}$ is the thermodynamic factor, $\mathfrak{R}_{0}$ is the equilibrium exchange rate of oxygen with the surface, and $D_{v}^{e f f}$ is the effective vacancy diffusivity (which includes any effects of path tortuosity for ion transport).

The derivation of this model assumes bulk vacancy diffusion. However, even in the case of surface transport, eqns, D-1 and D-2 may still hold, provided certain criteria are met: The surface and bulk must remain in rapid equilibrium, and the partition coefficient of vacancies between surface and bulk must be a constant. Based on the kinetics discovered and discussed in Section C (LSC thin films), these assumptions would appear to be valid ${ }^{[59]}$. In such case $D_{v}^{\text {eff }}$ represents an average effective parameter that depends on the relative coefficients and areas available for surface vs. bulk diffusion.

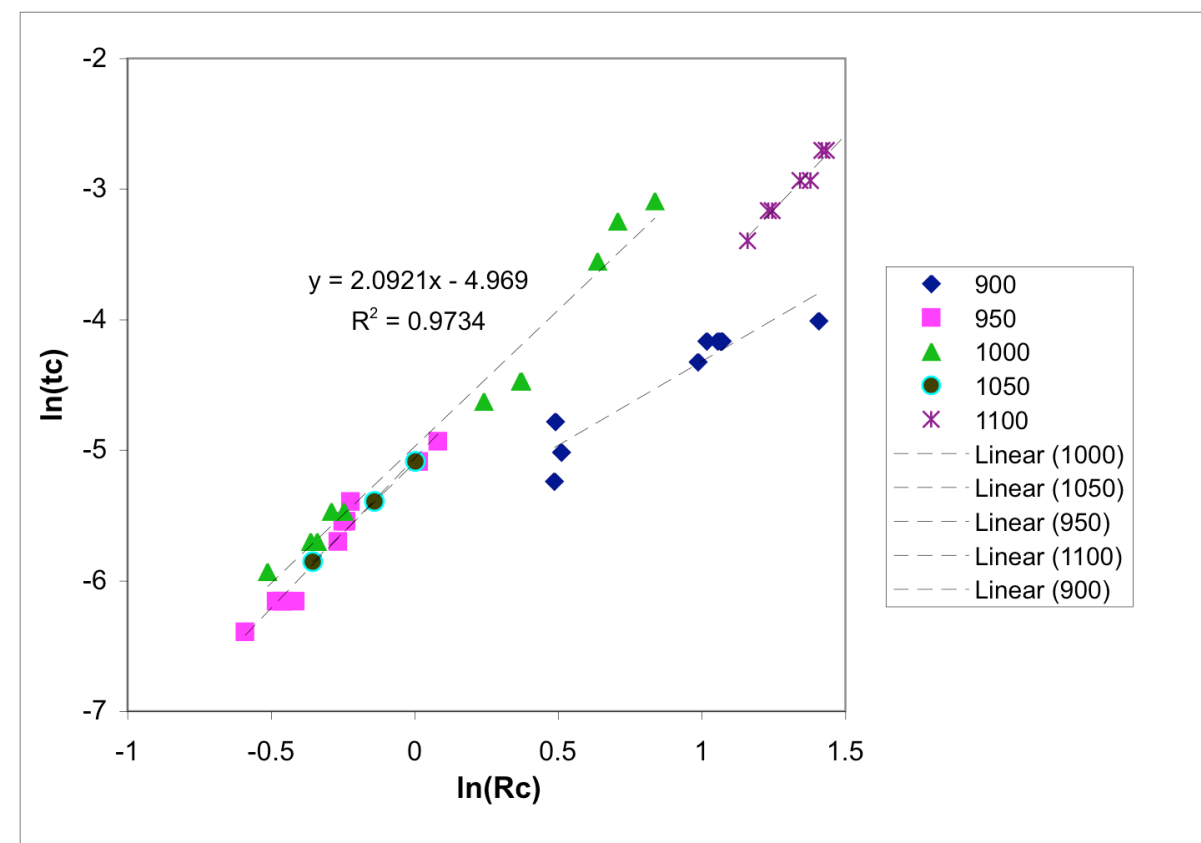

Fig. D-10. Parametric plot of $t_{c} v s$. $R_{c}$ for porous LSC-82, measured at $675^{\circ} \mathrm{C}$ in air, as a function of firing temperature and sample-to-sample variability. 


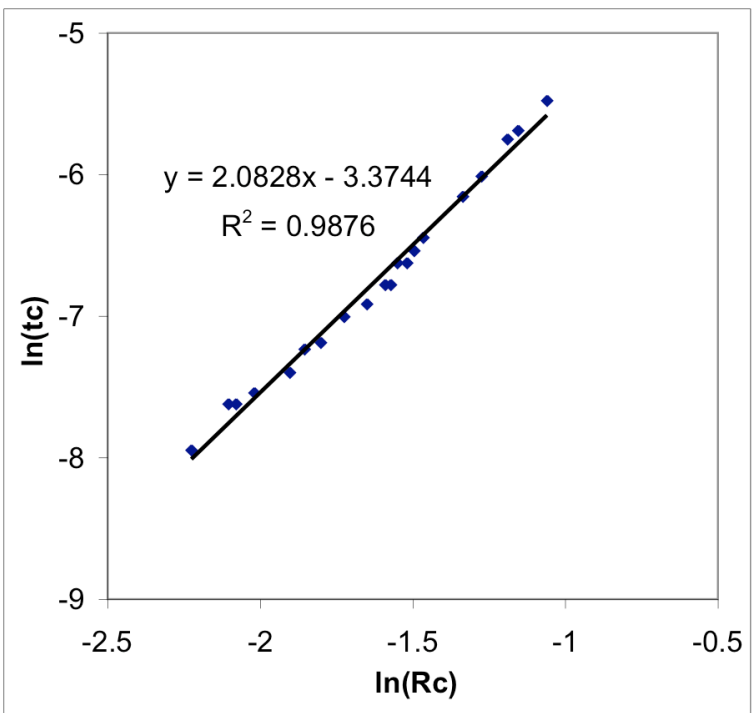

Fig. D-11. Parametric plot of $t_{c} v s . R_{c}$ for porous LSC-82, measured at $750^{\circ} \mathrm{C}$ in air, as a function of time (approximately 720 hours).

One consequence of co-limitation, as expressed by equations of D-1 and D-2, is that if we alter any of the parameters (individually), then $t_{c}$ and $R_{c}$ will scale with each other in specific ways related to the coupling between reaction and transport. For example, if we altered $D_{v}^{e f f}$ while leaving other parameters constant, $t_{c}$ would remain unchanged while $R_{c}$ varied. Likewise if we changed $\mathfrak{R}_{0}$, we would expect $t_{c}$ to scale as the square of $R_{c}$. Changes in porosity $(\varepsilon)$ would cause $t_{c}$ to scale as the square of $1 / R_{c}$.

Fig. D-10 shows how $t_{c}$ and $R_{c}$ scale with each other for LSC-82, measured at $650^{\circ} \mathrm{C}$ in air, both as a function of sample \# (sample-to-sample variability), and firing temperature. Other than the cell fired at $900^{\circ} \mathrm{C}$ (which we believe is not fully adhered to the electrolyte as explained in section D-8, below), the samples at any given firing temperature fall along a line (on a log-log plot) with slope $\sim 2.0 \pm 0.2$. Furthermore, except for the samples fired at the highest firing temperature $\left(1100^{\circ} \mathrm{C}\right)$, the data from multiple firing temperatures fall along the same line. The samples fired at $1100^{\circ} \mathrm{C}$ parallel this line, with values of $t_{\mathrm{c}}$ approximately a factor of 2 lower. Almost identical trends are observed at other testing temperatures between $625 \sim 725^{\circ} \mathrm{C}$ (tested in $25^{\circ} \mathrm{C}$ increments). At $750^{\circ} \mathrm{C}$ and above, the correlation starts to break down somewhat, but $t_{c}$ still generally scales more strongly than $R_{c}$ with respect to these variables. Similar trends are also observed for LSC-64.

Re-examination eqns. D-1 and D-2 shows that if the electrode is co-limited, the observed trends would be expected if either surface area $(a)$, exchange rate $\left(\Re_{0}\right)$, or both were subject to sample-to-sample variability. Furthermore, the fact that the trend holds for multiple firing temperatures suggests that these same factors are changing with the firing conditions.

Looking over the entire range of data in Fig. D-10, we can see that $t_{c}$ changes by a factor of about 50 from the best to the worst electrode. If this were due entirely to morphological variations, eqn. D-1 implies a 50-fold variation in surface area. However, the results of our microstructural analysis suggest that the surface area changes by less than a factor of 2 over these 
firing conditions. Also, sample-to-sample variability seems to generally outweigh the effect of firing temperature, with some cells exhibiting better performance at higher firing temperature (even though surface area is presumably lower). Together these trends point more strongly to $\mathfrak{R}_{0}\left(\mathrm{O}_{2}\right.$ exchange rate) rather than surface area as the more sensitive parameter to firing conditions and sample variation.

Further support for this view can be seen in Figure D-11, which shows how $t_{c}$ and $R_{c}$ scale with each other over time (about one month) as a sample degrades in performance. Again, a universal scaling of $t_{c} \sim R_{c}^{2}$ appears to hold. Although there is enormous variability in the rates of degradation (some samples degrade slowly while others quickly), this same $t_{c} \sim R_{c}^{2}$ correlation is always observed on a parametric plot.

According to Figure D-11, $t_{c}$ is changing by a factor of $\sim 10$ over the course of one month. Yet our microstructural studies show no evidence for any change in electrode morphology. This would seem to imply that it is the $\mathrm{O}_{2}$ exchange rate that is being poisoned (somehow) over time. This may, incidentally, explain the wide sample-to-sample variability, since the exact time at which the samples were tested was not precisely controlled, and thus the rapid degradation of some samples in the first $24 \sim 48$ hours may help explain some spread in the data.
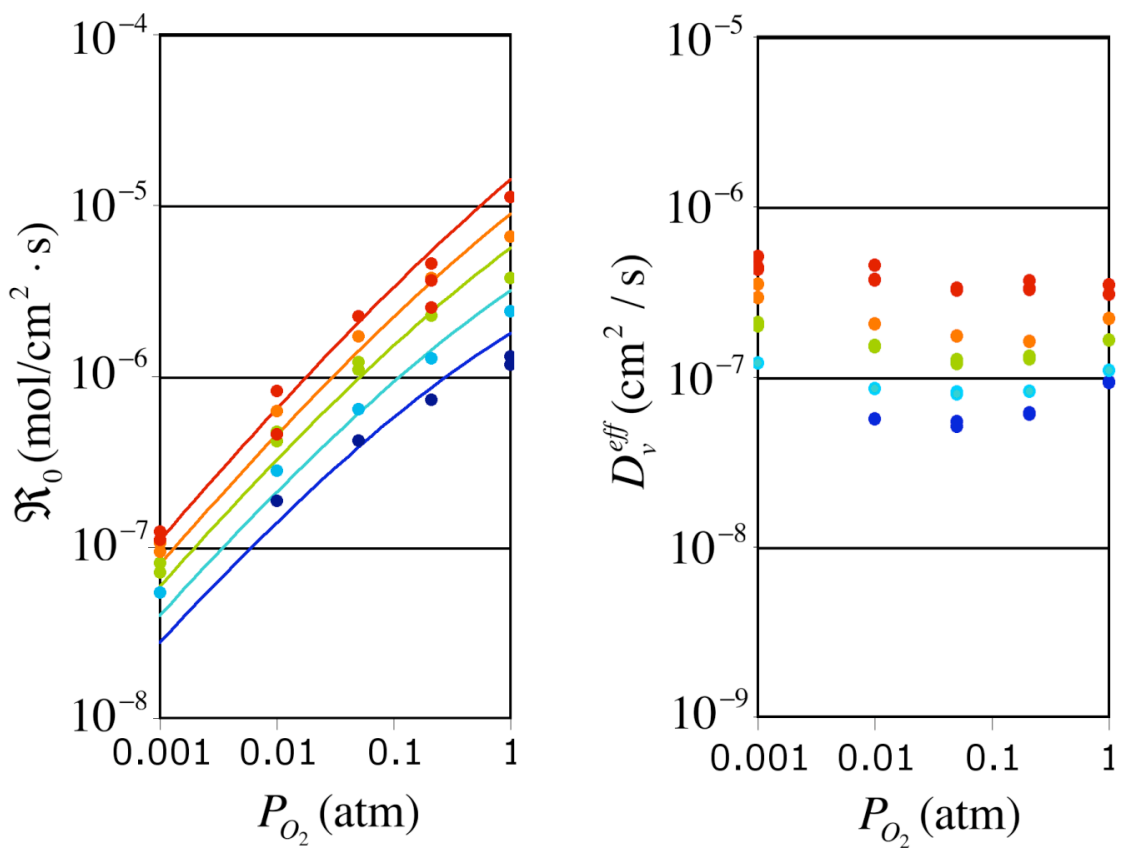

Fig. D-12. Plots of $\Re_{0}$ and $D_{v}^{e f f}$ vs. $P_{O 2}$, calculated using eqns. D-1 and D-2 based on measured values of $t_{c}$ and $R_{c}$ vs. $P_{O 2}$ for porous LSC-64 (fired at $1000^{\circ} \mathrm{C}$ ), measured at various temperatures from $650-750^{\circ} \mathrm{C}$ in $25^{\circ} \mathrm{C}$ increments from blue to red.

Another way to ascertain whether the electrode is co-limited is to examine the $P_{O 2^{-}}$ dependence of $t_{c}$ and $R_{c}$. One way to do this is to use eqns. D-1 and D-2 to extract values of $\Re_{0}$ and $D_{v}^{e f f}$ vs. $P_{O 2}$ from the data, and then compare the results to what we know or expect 
independently. As described elsewhere ${ }^{[42]}$, this calculation requires interpolation of existing oxygen nonstoichiometry data for $\delta\left(P_{\mathrm{O}_{2}}, T\right)$ and $\gamma\left(P_{\mathrm{O}_{2}}, T\right)$, for which we used a modification of Lankhorst's model ${ }^{[50]}$. Porosity and surface area parameters appearing in D-1 and D-2 were estimated based on our SEM results (minor errors in these parameters are not critical since they are independent of operating $T$ and $P_{\mathrm{O}_{2}}$ ).

Fig. D-12 shows the results of this calculation for LSC-64 (fired at $1000^{\circ} \mathrm{C}$ ), measured at various temperatures from $650-750^{\circ} \mathrm{C}$ in $25^{\circ} \mathrm{C}$ increments. As expected, the vacancy diffusion coefficient extracted from the data is only weakly $P_{O_{2}}$-dependent, with magnitudes on the order of $1 \times 10^{-7} \mathrm{~cm}^{2} / \mathrm{s}$ at $700^{\circ} \mathrm{C}$. This agrees within a factor of 2 of independent measurements of bulk diffusion in LSC-64 based on coulometric relaxation ${ }^{[60]}$. Meanwhile, the exchange rate exhibits roughly power-law dependence on $P_{O 2}$ with $\mathrm{n} \sim 0.5 \sim 0.6$, but with some curvature (increasing slope at lower $P_{O_{2}}$ ). As discussed elsewhere ${ }^{[42]}$, this behavior is entirely consistent with previous $\mathrm{O}_{2}$ exchange measurements on LSC in this range of conditions, including magnitude (within a factor of 10) and activation energy $(\sim 160 \mathrm{~kJ} / \mathrm{mol})$. The lines added to the graph show model predictions (fit within a scaling factor) based on the rate law determined from thin-film LSC (see Section C) which assumes dissociative adsorption is rate limiting. Thus the $T$ and $P_{O 2}$ dependence of the EIS data appear to be entirely consistent with a co-limited regime, with transport and kinetic properties agreeing well with independent measurements.

As a final consistency check, Fig. D-13 shows NLEIS data for LSC-64 half cells at $600^{\circ} \mathrm{C}$ and $650^{\circ} \mathrm{C}$, as a function of $P_{O 2}$. In these plots, the zero-frequency intercept of the $2^{\text {nd }}$, and $3^{\text {rd }}$ harmonic spectra are plotted against $\gamma$ (calculated from the $P_{O 2}$ as described above). The predictions of a co-limited model are also shown for comparison. This model is a nonlinear extension of the model in eqns. D-1 and D-2, based on the nonlinear dissociative adsorption model from Section $C^{[59]}$. In other words, this comparison tests both the co-limited model (by extending the comparison to include the nonlinear terms), as well as the applicability of the kinetics from the thin film measurements to a porous electrode of the same material.

The second harmonic, which exhibited a reasonably strong signal, is in general agreement in magnitude and trend. Unfortunately, the third harmonic is too small to be very clear, but the fact that it is small may simply reflect the nullification near $\gamma \sim-0.15$ predicted for these particular conditions. More $P_{\mathrm{O}_{2}}$-dependent data over a wider range of conditions would be needed to map out these curves more completely. Nonetheless, the NLEIS data appear to support the view that porous LSC is co-limited, and that the kinetics are governed by the same steps as for a thin film of the same material: dissociative adsorption.

A similar analysis of the $P_{O_{2}}$-dependence of LSC-82 electrodes was not possible due to lack of data in the right operating range. As stated previously, the correlation of $t_{c}$ and $R_{c}$ begins to deviate substantially from the predictions of co-limited behavior above about $700^{\circ} \mathrm{C}$. Unfortunately our $P_{O_{2}}$-dependent data was only acquired for LSC- 82 at $750^{\circ} \mathrm{C}$. We are currently working on the hypothesis that the increased temperature causes the electrode to move into a mixed regime, somewhere between co-limited and diffusion-limited. This is an interesting concept, as it implies oxygen reduction kinetics can be made fast enough under some conditions that it ceases to be a factor in the electrode resistance. 

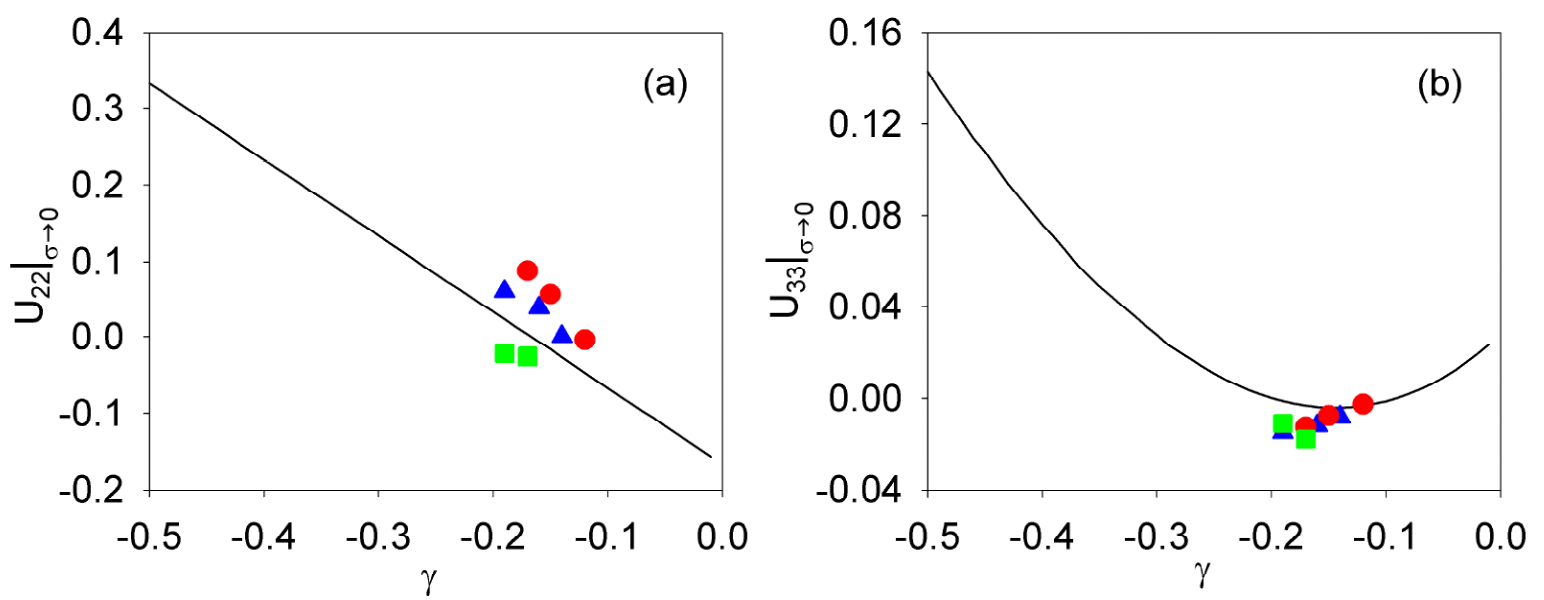

Figure D-13. NLEIS measurements on various LSC-64 half-cells. Values shown are the measured (data) and predicted (lines) zero-frequency limiting values of $\hat{U}_{2,2}$ (a) and $\hat{U}_{3,3}$ (b) as a function of $\gamma=\partial \ln \delta / \partial \ln P_{O_{2}}$, as calculated from $T$ and $P_{O 2}=0.01 \sim 0.21 \mathrm{~atm}$. Data shown are for a LSC-64 pellet cell, fired at $1100^{\circ} \mathrm{C}$, and tested at $600^{\circ} \mathrm{C}$ (blue triangles) and $650^{\circ} \mathrm{C}$ (red circles) and a LSC-64 microelectrode half cell fired at $1050^{\circ} \mathrm{C}$, and tested in air at $600^{\circ} \mathrm{C}$ and $650^{\circ} \mathrm{C}$ (green squares).

\section{D-8. Factors Influencing Interfacial Resistance}

The results above suggest that ion exchange at the electrolyte/mixed conductor interface ceases to be a factor when the electrode is fired at sufficiently high temperatures to achieve bonding. As exemplified by the data in Figure D-9, electrodes fired at lower temperatures $\left(<950^{\circ} \mathrm{C}\right)$ exhibit an additional impedance arc at high frequency, accompanied by increases in the impedance in general. The electrodes in this case were physically weak (easily scraped off with a thumbnail), and had inconsistent performance. In an attempt to determine what factors govern the quality of this interface, we studied the effects of electrolyte polishing on bonding. Our hypothesis was that if the electrolyte is polished, bonding will improve, and performance will be higher at lower firing temps. As shown below, this hypothesis was entirely wrong.

Rectangular plates of dense $\mathrm{Sm}$-doped ceria, $\mathrm{Ce}_{0.8} \mathrm{Sm}_{0.2} \mathrm{O}_{2-\delta}$ (SDC) were prepared as electrolytic substrates. These were fabricated by cutting and laminating green electrolyte tapes of composition $79 \% \mathrm{CeO}_{2} / 20 \% \mathrm{Sm}_{2} \mathrm{O}_{3} / 1 \% \mathrm{TiO}_{2}$ (Ceramatec, Salt Lake City), followed by sintering in air at $1650^{\circ} \mathrm{C}$ for 4 hours. Following fabrication, The phase composition of the sintered electrolyte was studied by X-ray diffraction (XRD) (Philips 1820). The surface of the sintered samples were treated as follows. For some samples, the surface was kept in initial state after sintering ("as-prepared" SDC). For a second group of samples, the surface was mechanically planarized using SiC paper No.600 ("ground" SDC). For a third group, some of the ground SDC samples were further polished using 1- $\mu \mathrm{m}$ diamond paste ("polished" SDC). The final group of samples was prepared by re-firing the polished SDC in air at different temperatures $(1450,1550$ and $1650^{\circ} \mathrm{C}$ ) for 4 hours. The surface morphology of the various SDC samples was examined using Scanning Electron Microscopy (SEM) (FEI Sirion), and the the surface composition of the 
electrolyte samples was studied by Electron Spectroscopy for Chemical Analysis (ESCA). For further details, see reference ${ }^{[61]}$.

For preparation of symmetric cells, both sides of the SDC plates were treated identically as described above. The electrodes (porous LSC-82) were applied by screen-printing and fired at $1080^{\circ} \mathrm{C}$ for 2 hours in air, as described previously. In order to determine if variations in polishing influence mechanical adhesion of the electrode, identically prepared samples were tested using mechanical scratching of the electrode using a sharp-edged piece of SDC ceramic. Electrochemical behavior of the symmetric cells was studied by EIS as described previously.

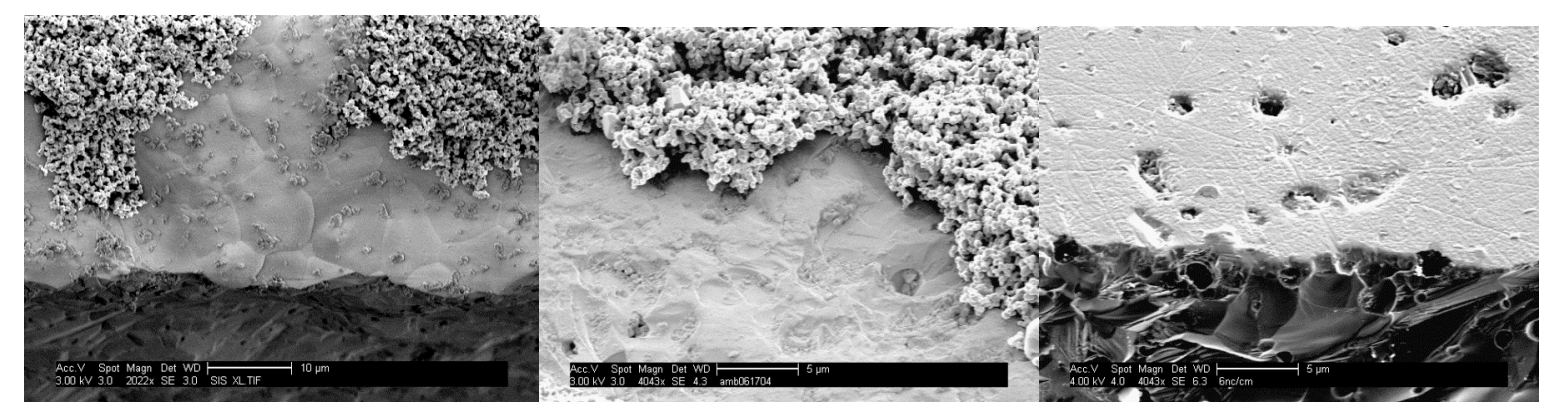

Fig. D-14. SEM micrographs of the SDC following electrode fabrication: as-prepared (left), ground (middle), and polished (right).

Fig. D-14 shows SEM micrographs of the as-prepared, ground, and polished SDC samples following electrode fabrication. The regions shown represent areas of the electrolyte surface that were only partially covered by the electrode (near the edge of the electrode screen pattern), or in areas where the electrode did not stick well, revealing the underlying electrolyte. For the asprepared electrolyte (left), the surface of the electrolyte has a granular morphology, with typical grain size of 5-10 $\mu \mathrm{m}$. As can be seen from the micrograph, the bulk of the electrolyte contains pores, but the granular layer formed on the surface during sintering is almost pore-free. XRD measurements of the initial (as-prepared) sintered electrolyte (prior to electrode application) showed presence of a single fluorite $\left(\mathrm{CeO}_{2}\right)$ phase. For the ground and polished samples (middle and right), the granular surface is removed, and the pores of the bulk are revealed at the surface. The surface of the ground SDC has a roughness of about $2 \mu \mathrm{m}$. For the polished SDC, the surface looks smooth, and 1-2 $\mu \mathrm{m}$ pores on the surface can be seen clearly in electrode-free areas. Based on our qualitative mechanical scratch test, it was found that electrodes fired on polished SDC can be scratched off easily (in fact, they can be wiped off with one's finger), but is very difficult to remove the electrodes (by scratching) from the as-prepared or ground substrates.

The composition of the surface of the various SDC samples was analyzed by ESCA, as was the bulk of the initial sample (fracture cross section). All spectra contained strong reflections from carbon together with the peaks corresponding to $\mathrm{Ce}, \mathrm{Sm}$ and $\mathrm{O}$. This result suggests that the gas-exposed surface is generally always covered with carbon-containing contaminants, presumably from $\mathrm{CO}_{2}$ adsorption (these materials are generally alkaline, and known to adsorb $\mathrm{CO}_{2}$ ). Carbon contamination from sources other than $\mathrm{CO}_{2}$ is unlikely since they were handled with gloves prior to analysis. For some samples, peaks identified as $\mathrm{Mo}, \mathrm{Ca}, \mathrm{Na}$ and $\mathrm{S}$ were also observed. Presence of Mo can be explained by the fact that the samples were fired in a furnace 
with Mo-containing heating elements and the surface could be contaminated during firing. In the case of bulk SDC (fracture cross-section), no element other than $\mathrm{Ce}, \mathrm{Sm}, \mathrm{C}$ and $\mathrm{O}$ were detected; therefore appearance of other elements on the surface may be due to contamination during processing, and/or due to accumulation of minor impurities from the electrolyte bulk on the surface during firing. The $\mathrm{Ce} / \mathrm{Sm}$ ratio, $r$, was also found to vary with polishing. Typically $r \sim$ $0.6 \pm 0.1$ on the as-prepared surface, while for the polished or ground surfaces $r \sim 1.7 \pm 0.2$. Heat treatment of the polished electrolyte results in Sm re-enrichment of the surface, with $r$-values for the re-fired samples at $1450-1650^{\circ} \mathrm{C}$ being close to that of the as-prepared SDC $(0.7 \pm 1)$.

Fig. D-15 shows complex impedance spectra for the LSC/SDC/LSC cells at $750^{\circ} \mathrm{C}$ with the as-prepared, ground and polished SDC (the plots are scaled to remove the ohmic contribution of the electrolyte). The shape of the spectra differs significantly for the various surface treatments. For the as-prepared surface, the spectrum contains one dominant arc, with a characteristic frequency of 50 200 Hz. However, for the ground and polished surfaces, a high frequency contribution is also present, apparent as a second distinct arc with a frequency of $2 \sim 3 \mathrm{kHz}$. The impedance magnitude increases with decreasing surface roughness: for the polished electrolyte the electrode impedance is more than 3 times that of the as-prepared electrolyte. For all cells, decreasing $P_{\mathrm{O} 2}$ results in increases in magnitude of the low frequency arc, while the high frequency arc remains nearly unchanged. The high frequency component of impedance which appears to grow with increasing surface polishing corresponds to a capacitance on the order of $10^{-5}-10^{-4} \mathrm{~F} / \mathrm{cm}^{2}$, which is typical for 2-D electrochemical interfaces ${ }^{[9]}$. Taking into account the order of capacitance, independence of $P_{\mathrm{O} 2}$ and sensitivity to the electrolyte surface preparation, it appears the high frequency arc is caused by charge transfer of oxygen ions between electrode (bulk or surface) and the electrolyte.
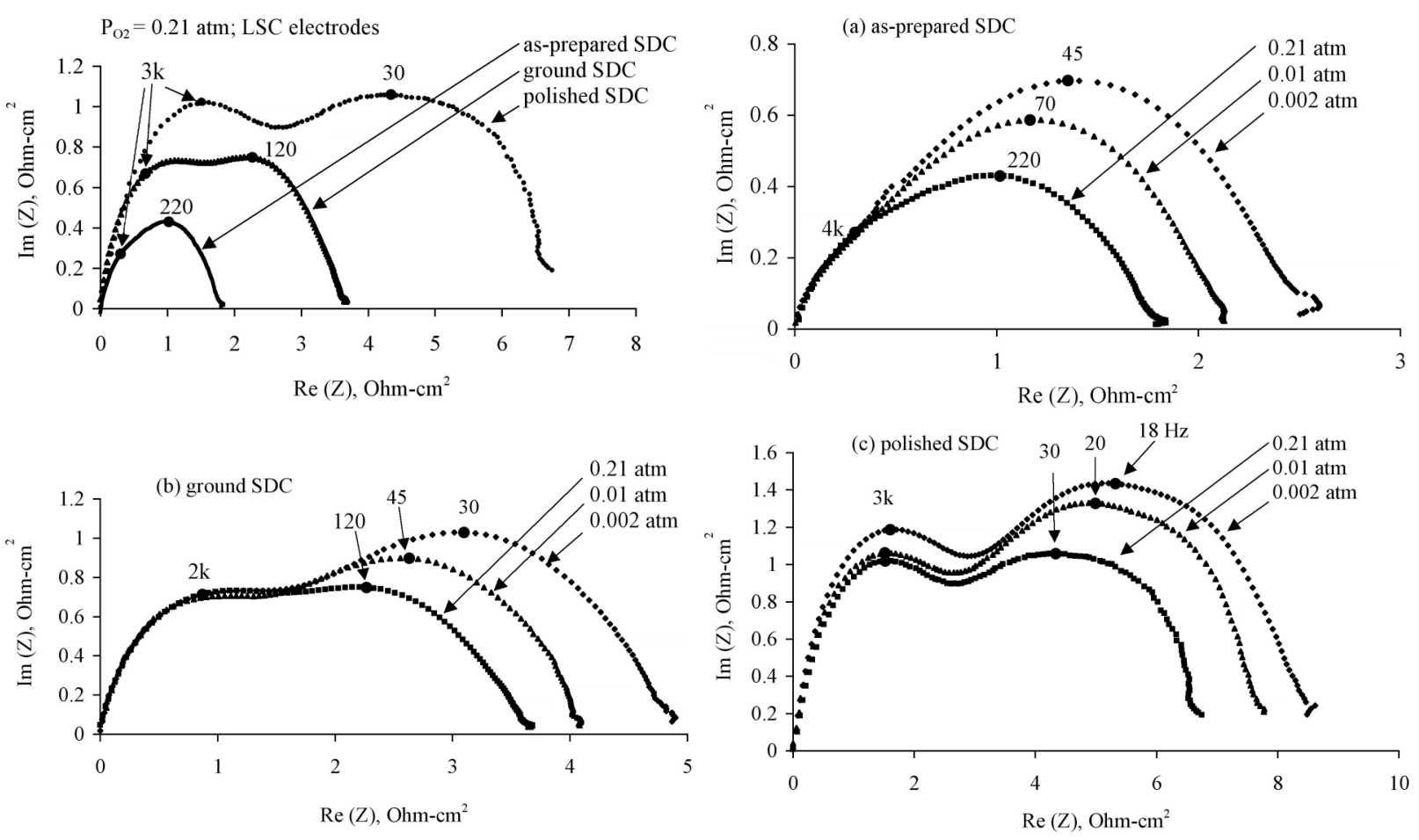

Fig. D-15. EIS spectra for the LSC/SDC/LSC cells with the as-prepared, ground and polished electrolyte, at $750^{\circ} \mathrm{C}$ as a function of $P_{O 2}$. 

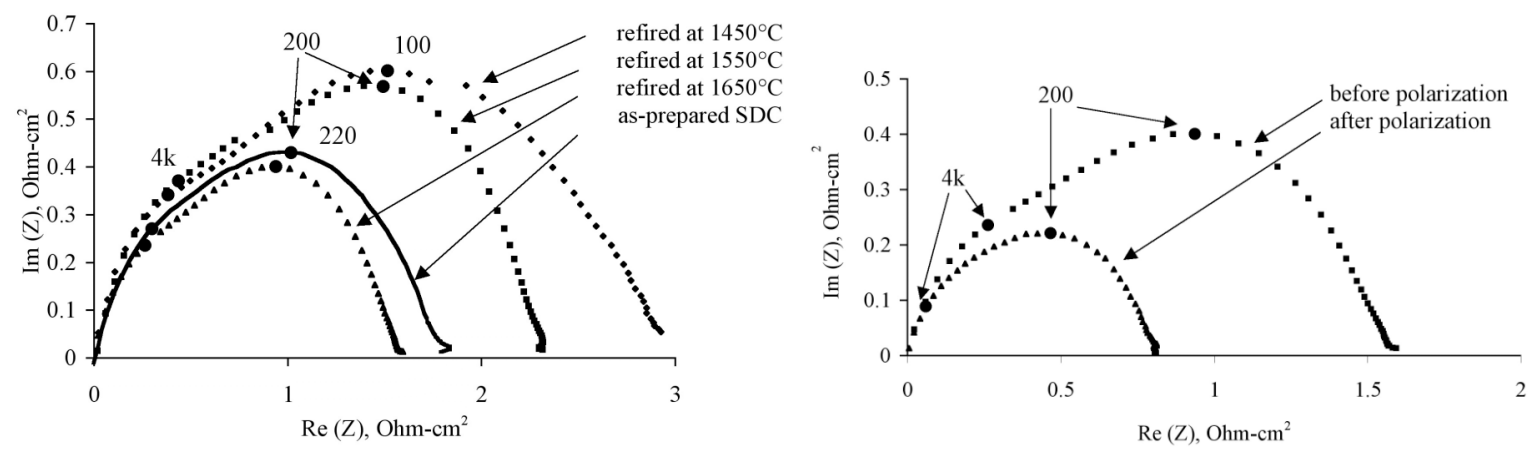

Fig. D-16. Left: EIS spectra for LSC/SDC/LSC cells comparing as-prepared electrolyte with re-fired polished electrolyte. Right: influence of polarization on the interfacial resistance. All measurements in air at $750^{\circ} \mathrm{C}$.

Thus our a.c. impedance data suggest that under the particular firing conditions we explored, the "natural" state of the as-prepared electrolyte is preferable for minimization of the electrode polarization losses. Interfacial resistance appears to be a minor contributor under "as-prepared" conditions (at a firing temperature of $1080^{\circ} \mathrm{C}$ ), but is significantly apparent for the ground and polished samples.

Based on these observations, one might expect re-firing of the polished electrolyte at high temperature (resulting in restoration of the surface to its as-prepared state) to give an improvement in the electrode performance. Impedance spectra of the LSC/SDC/LSC cells with the polished and then re-fired electrolyte measured at $750^{\circ} \mathrm{C}$ in air are given in D-16 (left). Firing of the polished electrolyte even at relatively low temperature $\left(1450^{\circ} \mathrm{C}\right)$ results in improvement of the electrode resistance by a factor of 2 or more, while increasing of temperature to $1650^{\circ} \mathrm{C}$ recovers the performance of the as-prepared electrolyte.

Although one can see clear morphological differences among the various electrolyte surfaces shown in Fig. D-14, the overall morphology remains relatively flat, with an undulation of $\sim 1$ micron. This situation is quite different than, for example, the case where porous layers are intentionally added to the electrolyte surface for the purpose of increasing surface area and/or creating an interlocking matrix for support of a relatively fragile electrode material. If anything, the ground samples appear to have a somewhat higher surface area, and yet lower performance. Thus we think it unlikely that decreased surface area is the cause of increased interfacial resistance for the treated samples.

An alternative theory is that the unique composition of the SDC surface is critical in determining the degree and quality of bonding between the two materials. This is consistent with our observation that the electrode could easily be wiped off the SDC electrolyte that had been polished, exposing fresh bulk material. Although it is intuitive that the as-prepared and ground samples might provide a slightly more interlocking (and therefore scratch-resistant interface), we did not observe a clear trend among surface undulation, adhesion, and interfacial resistance. In contrast, the chemistry of the surface is expected to have a significant impact on how the materials react and interdiffuse during bonding, as well as the structure of the resulting interface. However, it remains unclear what factors govern the chemistry of bonding. One 
possibility, which we would currently like to investigate, is that minor impurities on the as-fired electrolyte surface play an important role in bonding. Small amount of Si, or other inorganic binding agents (not intentional, yet ubiquitous in ceramic processing nonetheless) might be critical in helping to establish bonding during electrode processing. When the samples are polished or ground, these impurities would be removed, possibly making it difficult to form a reversible interface. Upon re-firing the electrolyte at temperatures high enough to sinter, these impurities might become mobile again, emerge from grain boundaries, re-establishing the native surface.

\section{D-9. Summary}

The analysis of any one impedance spectrum in terms of mechanism is fraught with difficulties, and seldom yields unique or meaningful results. However, we have shown here that even with very distorted, non-ideal spectra, it is possible to probe mechanism by examining the gross scaling factors describing the impedance (frequency and magnitude) over a wide enough range of parameter space. In particular, we emphasize the importance of examining the frequency response as well as the resistance, and how these characteristics scale with each other. We also emphasize the importance of physical models (rather than equivalent circuits) in gaining a meaningful understanding of reaction mechanism.

Unlike LSC on YSZ, at sufficiently high firing temperatures $\left(\geq 1000^{\circ} \mathrm{C}\right)$, LSC electrodes on GDC/SDC exhibit little or no interfacial resistance - i.e. charge-transfer is reversible between electrode and electrolyte materials. This appears to be a unique feature of these electrodes, as distinct from more traditional materials where charge-transfer at the TPB is dominant (LSM/YSZ). Pre-polishing of the electrolyte prior to electrode firing increases interfacial resistance, suggesting that impurities or grain-boundary phases present on the electrolyte may be important in establishing a reversible bond during electrode processing.

Given a reversible LSC/ceria interface, performance of LSC electrodes appears to be colimited by $\mathrm{O}_{2}$ reduction on LSC and ionic transport on/within LSC to the electrolyte. This conclusion is supported by numerous observations, including a strong $2^{\text {nd }}$-order correlation between electrode frequency and electrode resistance that spans a wide range of conditions $\left(P_{O 2}\right.$, $T$, Sr-doping, time, polarization), consistency with nonlinear NLEIS response, and a close match of the impedance data to independent properties based on a co-limited model. The co-limited model begins to break down at higher temperatures $\left(\mathrm{T} \geq 725^{\circ} \mathrm{C}\right)$, which may reflect a shift toward a bulk-diffusion-limited regime.

Above a firing temperature of $\sim 1000^{\circ} \mathrm{C}$, electrode resistance increases significantly with increased firing temperature. BET measurements, as well as recent analysis of the electrode morphology using FIB-SEM show only minor changes in morphology over the same range of processing conditions. Quantitative analysis of electrode frequency and resistance as a function of firing conditions shows that these changes are most likely related to the changes in kinetics at the surface. Some reasons why the surface chemistry might be negatively influenced by increased firing temperature were discussed.

Examination of electrode frequency and resistance as a function of time suggests that the primary contributor to electrode degradation are changes to the $\mathrm{O}_{2}$ exchange rate at the surface. Likewise, significant sample-to-sample variation in performance also appears to be tied most 
closely to differences in $\mathrm{O}_{2}$ exchange rate. Thus (as seen with firing conditions), it appears to be the surface chemistry that is the most sensitive factor in governing performance. It remains unclear what changes are occurring to the surface on an atomic level, or why these changes are so sensitive to the details of processing and operation. We recommend further work that focuses on electrodes of well-defined or characterized geometry, and probes the details of surface structure, composition, and impurities.

\section{References}

1. Nagata, M.; Itoh, Y.; Iwahara, H., Solid State Ionics 1994, 67, 215-224.

2. Hsieh, G.; Mason, T. O.; Garboczi, E. J.; Pederson, L. R., Solid State Ionics 1997, 96, (34), 153-172.

3. Winkler, J.; Hendriksen, P. V.; Bonanos, N.; Mogensen, M., Journal of the Electrochemical Society 1998, 145, (4), 1184-1192.

4. Adler, S. B., J. Electrochem. Soc. 2002, 149, (5), E166-E172.

5. Hashibon, A.; Raz, S.; Riess, I., Solid State Ionics 2002, 149, (3-4), 167-176.

6. Newman, J. S.; Thomas-Alyea, K. E., Electrochemical Systems. 3rd ed.; Wiley: 2004.

7. Dunyushkina, L. A.; Lu, Y. X.; Adler, S. B., Journal of the Electrochemical Society 2005, 152, (8), A1668-A1676.

8. Thermal Expansion - Nonmetallic Solids. IFI/Plenum Data Company: 1977; Vol. 13.

9. Adler, S. B., Chemical Reviews 2004, 104, (10), 4791-4843.

10. Adler, S. B., Solid State Ionics 1998, 111, (1,2), 125-134.

11. Eguchi, K.; Setoguchi, T.; Inoue, T.; Arai, H., Solid State Ionics 1992, 52, (1-3), $165-$ 172.

12. Jiang, S. P.; Love, J. G.; Apateanu, L., Solid State Ionics 2003, 160, (1-2), 15-26.

13. Yang, Y.; Jacobson, A. J.; Chen, C. L.; Luo, G. P.; Ross, K. D.; Chu, C. W., Applied Physics Letters 2001, 79, (6), 776-778.

14. Darowicki, K., Corrosion Science 1995, 37, (6), 913-925.

15. Medina, J. A.; Schwartz, D. T., Journal of the Electrochemical Society 1997, 144, (1), $155-164$.

16. Medina, J. A.; Schwartz, D. T., Physics of Fluids 1996, 8, (11), 2895-2905.

17. Wilson, J. R.; Adler, S. B.; Schwartz, D. T., Physics of Fluids 2005, 17, (6).

18. Bosch, R. W.; Bogaerts, W. F., Corrosion 1996, 52, (3), 204-212.

19. Marucci, L., Optics and Lasers in Engineering 2002, 37, 601.

20. Shan, J.; Nahata, A.; Heinz, T. F., Journal of Nonlinear Optical Physics \& Materials 2002, 11, (1), 31-48. 
21. Mao, Z. S., International Journal of Heat and Mass Transfer 1995, 38, (14), 2667-2675.

22. Singhal, P.; Kawagoe, K. T.; Christian, C. N.; Kuhr, W. G., Analytical Chemistry 1997, $69,(8), 1662-1668$.

23. Wilson, J. R.; Schwartz, D. T.; Adler, S. B., Electrochemica Acta 2004, submitted.

24. Adler, S. B.; Lane, J. A.; Steele, B. C. H., J. Electrochem. Soc. 1996, 143, (11), 35543564.

25. Simner, S. P.; Bonnett, J. R.; Canfield, N. L.; Meinhardt, K. D.; Shelton, J. P.; Sprenkle, V. L.; Stevenson, J. W., Journal of Power Sources 2003, 113, (1), 1-10.

26. Simner, S. P.; Anderson, M. D.; Pederson, L. R.; Stevenson, J. W., Journal of the Electrochemical Society 2005, 152, A1851-A1859.

27. Huff, S. Diagnosis and Characterization of Gas Diffusion and Grain Boundary Processes in Solid Oxide Fuel Cell Systems Using Linear and Non-linear Impedance Spectroscopy and Electrochemical Modeling. M.S., University of Washington, Seattle, 2007.

28. Sunde, S.; Nisancioglu, K.; Gur, T. M., Journal of the Electrochemical Society 1996, 143, (11), 3497-3504.

29. ten Elshof, J. E.; Lankhorst, M. H. R.; Bouwmeester, H. J. M., Journal of the Electrochemical Society 1997, 144, (3), 1060-1067.

30. Lankhorst, M. H. R.; Bouwmeester, H. J. M., Journal of the Electrochemical Society 1997, 144, (4), 1261-1267.

31. Diethelm, S.; Closset, A.; Van Herle, J.; McEvoy, A. J.; Nisancioglu, K., Solid State Ionics 2000, 135, (1-4), 613-618.

32. Bredesen, R.; Mertins, F.; Norby, T., Catalysis Today 2000, 56, (1-3), 315-324.

33. Lane, J. A.; Kilner, J. A., Solid State Ionics 2000, 136, 997-1001.

34. Wang, S.; Verma, A.; Yang, Y. L.; Jacobson, A. J.; Abeles, B., Solid State Ionics 2001, 140, (1-2), 125-133.

35. Preis, W.; Bucher, E.; Sitte, W., Journal of Power Sources 2002, 106, (1-2), 116-121.

36. van der Haar, L. M.; den Otter, M. W.; Morskate, M.; Bouwmeester, H. J. M.; Verweij, H., Journal of the Electrochemical Society 2002, 149, (3), J41-J46.

37. Yoo, J.; Verma, A.; Wang, S. Y.; Jacobson, A. J., Journal of the Electrochemical Society 2005, 152, (3), A497-A505.

38. Chater, R. J.; Carter, S.; Kilner, J. A.; Steele, B. C. H., Solid State Ionics 1992, 53-56, 859-867.

39. van Doorn, R. H. E.; Fullarton, I. C.; de Souza, R. A.; Kilner, J. A.; Bouwmeester, H. J. M.; Burggraaf, A. J., Solid State Ionics 1997, 96, 1-7.

40. De Souza, R. A.; Chater, R. J., Solid State Ionics 2005, 176, (23-24), 1915-1920.

41. De Souza, R. A., Physical Chemistry Chemical Physics 2006, 8, (7), 890-897.

42. Adler, S. B.; Wilson, J. R.; Chen, X. Y., Journal of Catalysis 2007, 245, 91-109. 
43. Yang, Y. L.; Chen, C. L.; Chen, S. Y.; Chu, C. W.; Jacobson, A. J., Journal of the Electrochemical Society 2000, 147, (11), 4001-4007.

44. Endo, A.; Fukunaga, H.; Wen, C.; Yamada, K., Solid State Ionics 2000, 135, (1-4), 353358.

45. Kawada, T.; Suzuki, J.; Sase, M.; Kaimai, A.; Yashiro, K.; Nigara, Y.; Mizusaki, J.;

Kawamura, K.; Yugami, H., Journal of the Electrochemical Society 2002, 149, (7), E252E259.

46. Sase, M.; Ueno, D.; Yashiro, K.; Kaimai, A.; Kawada, T.; Mizusaki, J., Journal of Physics and Chemistry of Solids 2005, 66, (2-4), 343-348.

47. Baumann, F. S.; Fleig, J.; Habermeier, H. U.; Maier, J., Solid State Ionics 2006, 177, 1071-1081.

48. Wilson, J. R.; Schwartz, D. T.; Adler, S. B., Electrochimica Acta 2006, 51, (8-9), 13891402.

49. Mizusaki, J.; Mima, Y.; Yamauchi, S.; Fueki, K.; Tagawa, H., Journal of Solid State Chemistry 1989, 80, 102-111.

50. Lankhorst, M. H. R.; Bouwmeester, H. J. M.; Verweij, H., Physical Review Letters 1996, 77, (14), 2989-2992.

51. Lankhorst, M. H. R.; Bouwmeester, H. J. M.; Verweij, H., Journal of Solid State Chemistry 1997, 133, (2), 555-567.

52. Bauerle, J. E., Journal of Physical Chemistry: Solids 1969, 30, 2657-2670.

53. Fabry, P.; Kleitz, M.; Deportes, C., Journal of Solid State Chemistry 1972, 5, (1), 1-10.

54. Gerischer, H., Z. Phys. Chem. 1951, 198, 216.

55. Wilson, J. R.; Kobsiriphat, W.; Mendoza, R.; Chen, H. Y.; Hiller, J. M.; Miller, D. J.; Thornton, K.; Voorhees, P. W.; Adler, S. B.; Barnett, S. A., Nature Materials 2006, 5, (7), 541-544.

56. Berthier, F.; Diard, J.-P.; Le Gorrec, B.; Montella, C., Corrosion 1995, 51, 105-115.

57. Mitterdorfer, A.; Gauckler, L. J., Solid State Ionics 1999, 117, (3-4), 187-202.

58. Mitterdorfer, A.; Gauckler, L. J., Solid State Ionics 1999, 117, (3-4), 203-217.

59. Wilson, J. R. PhD Thesis. University of Washington, Seattle, 2007.

60. Lankhorst, M. Thermodynamic and transport properties of mixed ionic-electronic conducting perovskite-type oxides. Univerdsity of Twente, Enschede , Netherlands, 1997.

61. Dunyushkina, L. A.; Adler, S. B., Journal of the Electrochemical Society 2005, 152, (10), A2040-A2045. 